\title{
EFFICIENCY PERFORMANCE IMPROVEMENT USING PARALLEL DC-DC CONVERTERS WITH A DIGITAL CONTROLLER
}

\author{
A Thesis \\ presented to \\ the Faculty of California Polytechnic State University, \\ San Luis Obispo
}

\author{
In Partial Fulfillment \\ of the Requirements for the Degree \\ Master of Science in Electrical Engineering \\ by \\ Daniel Paul Forbes
}

May 2012 
(C) 2012

Daniel Paul Forbes

ALL RIGHTS RESERVED 


\section{COMMITTEE MEMBERSHIP}

TITLE:

Efficiency Performance Improvement Using Parallel DC-DC Converters with a Digital Controller

AUTHOR:

Daniel Paul Forbes

DATE SUBMITTED:

May 2012

COMMITTEE CHAIR:

Dr. Taufik, Professor

COMMITTEE MEMBER: Dr. Dale Dolan, Assistant Professor

COMMITTEE MEMBER: Dr. Xiao-Hua (Helen) Yu, Professor 


\begin{abstract}
Efficiency Performance Improvement Using Parallel DC-DC Converters with a Digital Controller Daniel Paul Forbes
\end{abstract}

A system to improve efficiency performance of a DC-DC converter is simulated and built. The proposed system combines multiple DC-DC converters in parallel and implements a digital control scheme and load-share controller. A model of the system is developed in MATLAB Simulink and the model demonstrates the improved converter's efficiency particularly at low load conditions. This simulation is then designed into a hardware system running three DC-DC converters in parallel, controlled by a microcontroller and a load-share controller. The hardware also confirms the simulation results, although some hardware refinements are evident as simulation results are superior. The system is designed to be scalable in the number of converters and the total output power, as well as being DC-DC converter topology-independent. Simulation results show the system maintaining better than $88 \%$ efficiency over almost $90 \%$ of the load range of the system. This system could be implemented where dynamic loads typically occur, such as in electric vehicle charging. 


\section{ACKNOWLEDGMENTS}

Thank you to my parents for their continued support and encouragement, and to my employer for giving me the time off to complete this project. 


\section{TABLE OF CONTENTS}

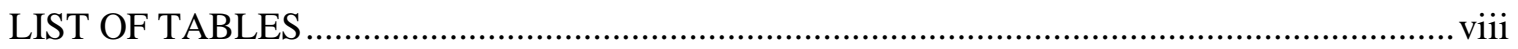

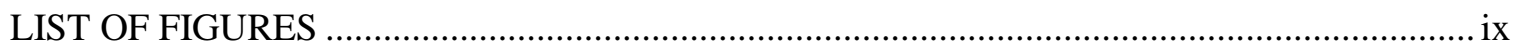

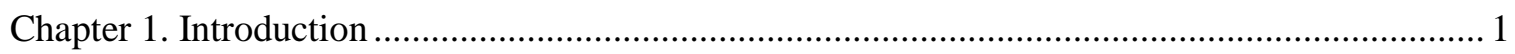

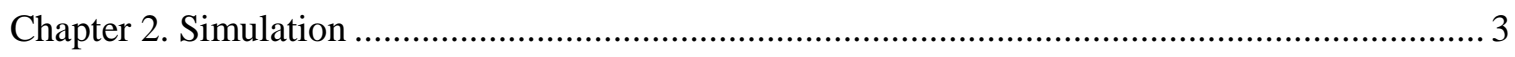

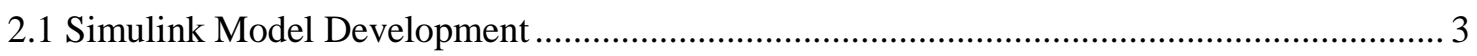

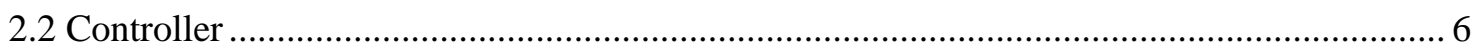

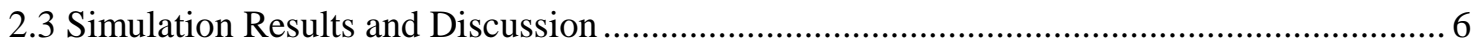

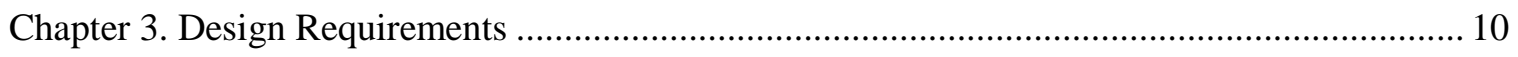

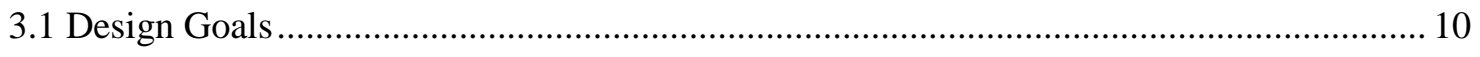

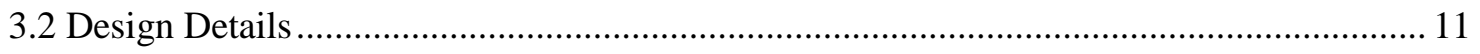

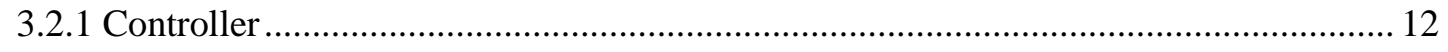

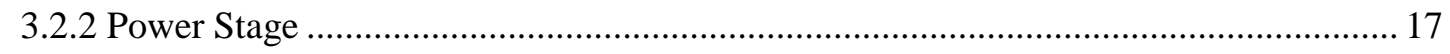

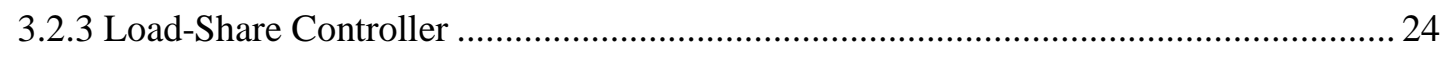

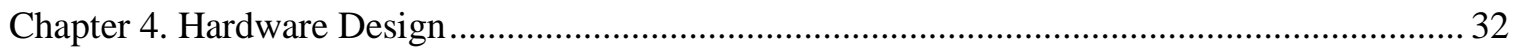

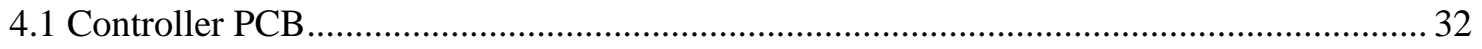

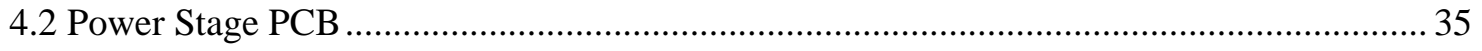

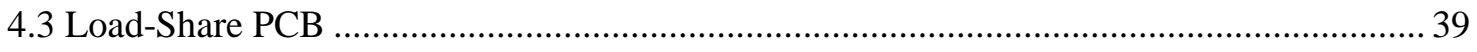

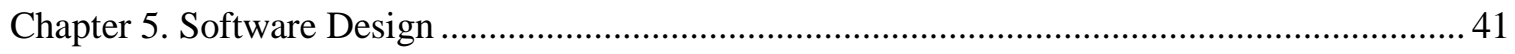

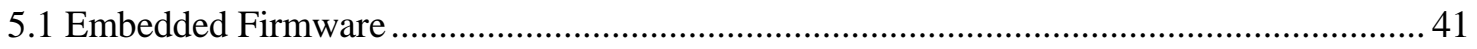

5.1.1 Analog-to-Digital Converter (ADC) Configuration ............................................... 41

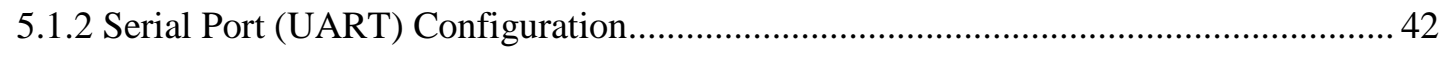

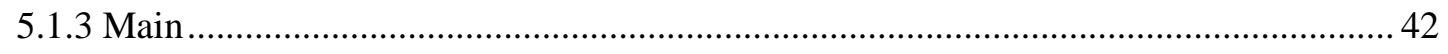

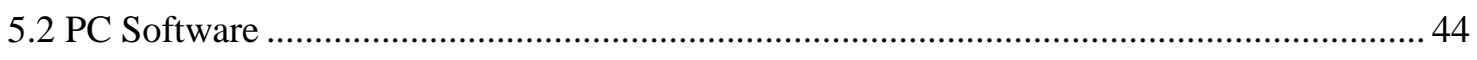

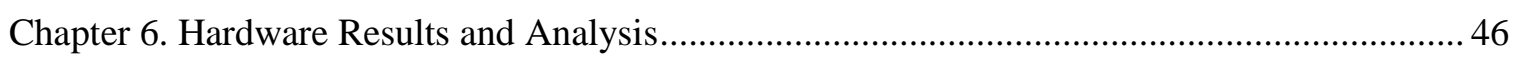




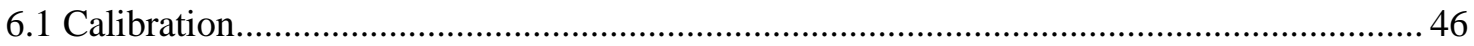

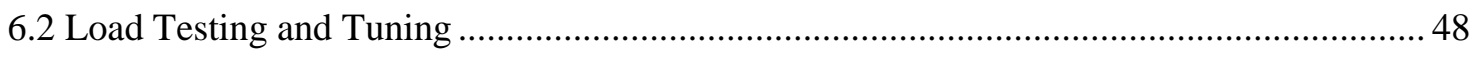

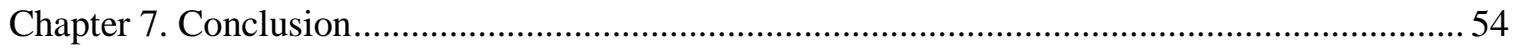

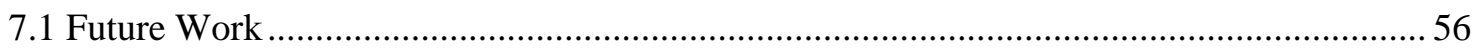

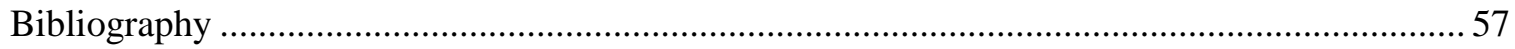

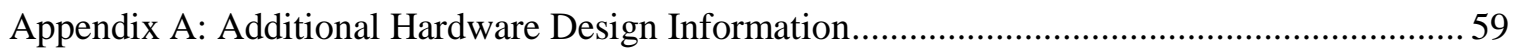

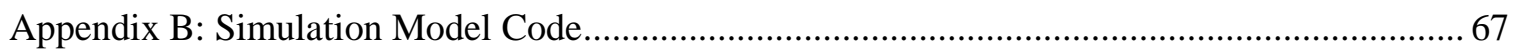

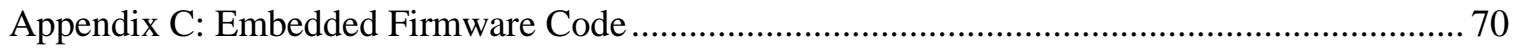

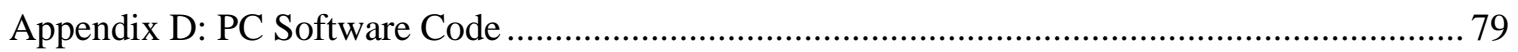




\section{LIST OF TABLES}

Table 3-1. Resistor Divider Voltage Set Values ..................................................................... 14

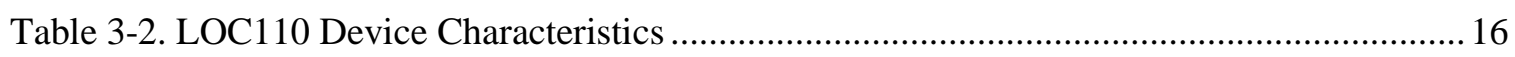

Table 3-3. PTEA415050N2AD Input/Output Characteristics ............................................... 17

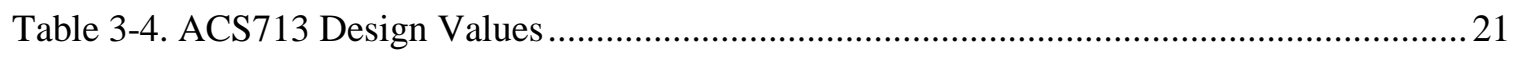

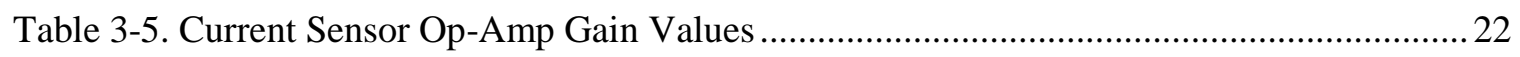

Table 3-6. Test Parameters Used To Find Unity Gain Crossover Frequency of

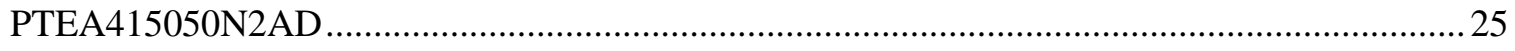

Table 3-7. Unity Gain Crossover Frequency Results ............................................................ 27

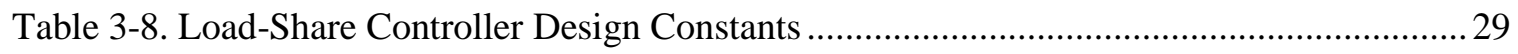

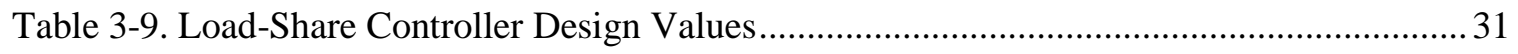

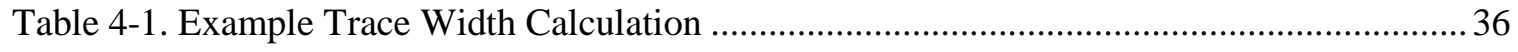

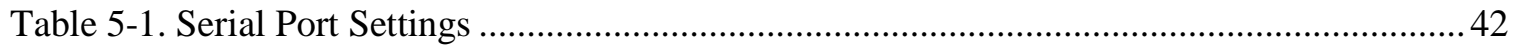

Table 6-1. Test Equipment Used for System Analysis ........................................................... 46

Table 6-2. LOC110 Linear Optocoupler Linearity Check …...................................................... 47

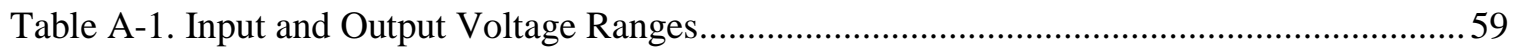

Table A-2. Calculation Results for Input and Output Voltage Sense Resistor Dividers ...............59

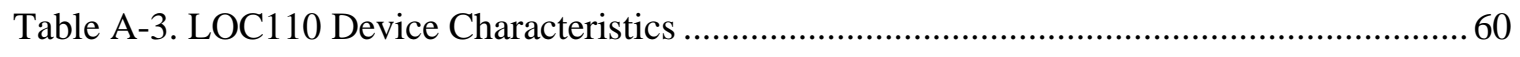

Table A-4. Calculation Results for LOC110 Support Circuitry .............................................. 60 


\section{LIST OF FIGURES}

Figure 2-1. DC-DC Converter Efficiency Model …................................................................. 3

Figure 2-2. Complete Parallel-Connected DC-DC Converter Simulink Model ..............................5

Figure 2-3. Load Current vs. Efficiency of the PTEA415050N2AD DC-DC .............................. 7

Figure 2-4. Load Current vs. Efficiency of the UCH-5/30-D48N DC-DC .................................... 8

Figure 2-5. Simulation Output Showing Efficiency Curves ................................................

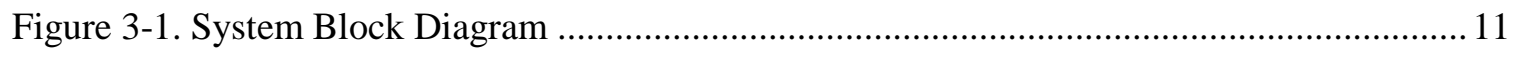

Figure 3-2. Power Distribution and Reference Voltages ...................................................... 12

Figure 3-3. Microcontroller and Support Circuitry ............................................................ 13

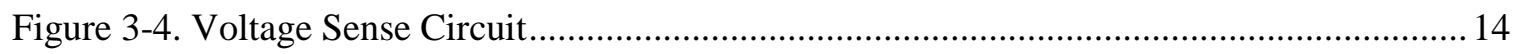

Figure 3-5. Efficiency vs. Output Current for the PTEA415050N2AD ...................................... 18

Figure 3-6. Standard Application Circuit for the PTEA415050N2AD....................................... 19

Figure 3-7. Isolated Enable Circuit for PTEA415050N2AD .................................................. 19

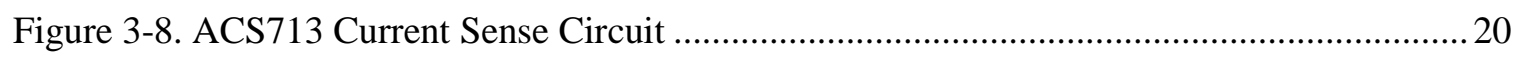

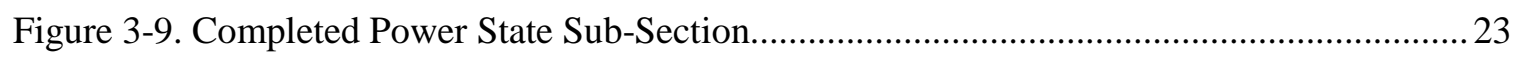

Figure 3-10. Setup to Measure Unity Gain Crossover Frequency of DC-DC ….........................25

Figure 3-11. Unity Gain Crossover Frequency Bode Plot: Unit 1 .........................................26

Figure 3-12. Unity Gain Crossover Frequency Bode Plot: Unit 2 …..................................... 26

Figure 3-13. Unity Gain Crossover Frequency Bode Plot: Unit 3 .......................................... 27

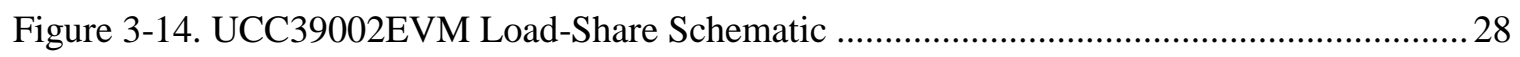

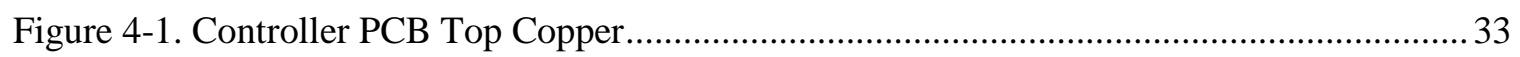

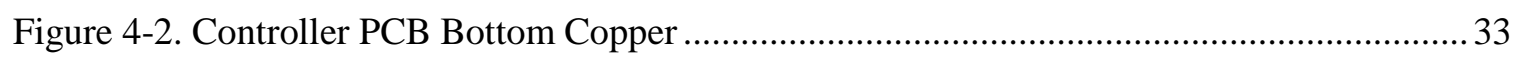

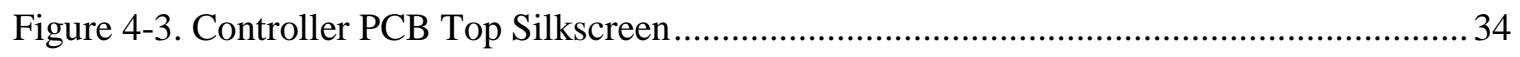

Figure 4-4. Controller PCB Bottom Silkscreen (Mirrored) ...................................................... 34 
Figure 4-5. Control PCB Photo with Features Labeled

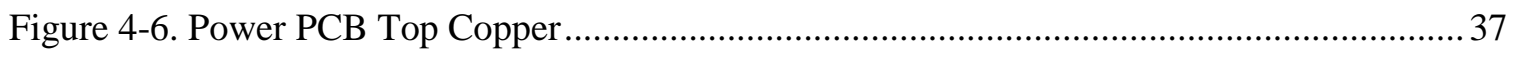

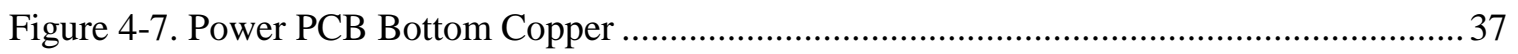

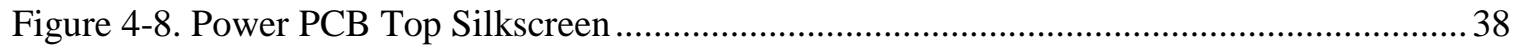

Figure 4-9. Power PCB Bottom Silkscreen (Mirrored) …....................................................... 38

Figure 4-10. Power PCB Photo with Features Labeled …......................................................... 39

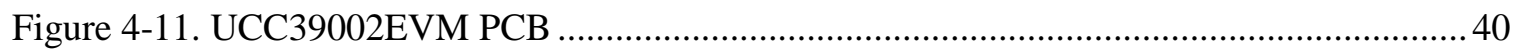

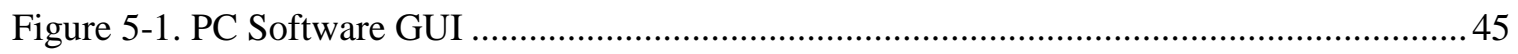

Figure 6-1. LOC110 Linear Optocoupler Linearity Calibration .................................................. 47

Figure 6-2. Load Currents of Each DC-DC Converter During Full-Load Test ............................. 48

Figure 6-3. Efficiency Curve for Generated from Full-Load Test ............................................. 49

Figure 6-4. Single DC-DC Converter Used as Control Case ......................................................50

Figure 6-5. Complete Digitally-Controlled Parallel DC-DC Converter System ...........................51

Figure 6-6. Test Bench Setup with Thesis System Attached ....................................................52

Figure 6-7. Testing Single DC-DC Converter (Control Case)....................................................53

Figure 7-1. Efficiency Curves from Simulation and Hardware ..................................................55

Figure A-1. Controller Schematic - 5V, 3.3V, Non-Isolated 5V, Connector, and Test Points ..... 61

Figure A-2. Controller Schematic - USB, ISCP, Microcontroller, GPIO LEDs and Buttons....... 62

Figure A-3. Controller Schematic - Input and Output Voltage Sense Circuits .............................63

Figure A-4. Power Schematic - Test Points, Connectors, and RAIL 1 ....................................... 64

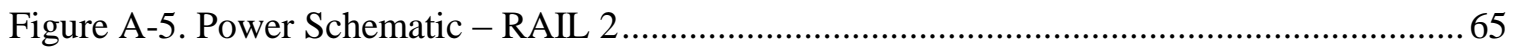

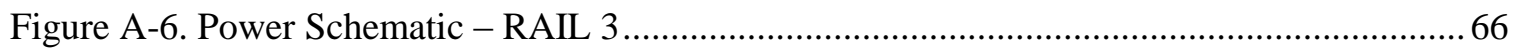




\section{Chapter 1. Introduction}

In the developed world today there exist many technologies which rely on DC-DC converters. These technologies range from the simple cell phone charger and desktop computer power supply to large electric vehicle chargers and DC power distribution stations. Within all of these DC-DC converters there exist known inefficiencies which waste power during the use of the converter. Each converter is designed with a specific load current range in mind, and the efficiency is optimized over this load range. While the efficiency of DC-DC converters has improved recently, most still exhibit a drop-off in efficiency as the load current decreases.

With larger and larger DC-DC converters being designed and used in industry, the main components (inductors, capacitors, switches, diodes, etc.) become increasingly expensive and cumbersomely large. As these converters are becoming more wide-spread, they are required to perform continuously without failures or faults, sometimes in harsh environmental conditions. These shortcomings of the DC-DC converter are what led to the development of systems employing parallel-connected DC-DC converters. These systems offer several advantages over single DC-DC converters, including increased reliability, decreased stress on critical components, improved efficiency, and increased flexibility $[1,2]$.

While paralleling converters has its advantages, care must be taken in designing a system due to the fact that DC-DC converters of the same model will not necessarily be identical [3]. Because of these differences between converters, a load-share scheme must be implemented to ensure equal currents through all converters. The literature shows that there are many different methods for load-share control, including passive and active control, and master-slave and master-less configurations, all of which have advantages and disadvantages $[3,2]$. 
In distributed power systems, parallel-connected converters have been in use for many years [2]. In the emerging market of electric vehicles, there arises the need for high-power chargers which are reliable, cost effective, and very efficient [4]. These chargers most often use a constantcurrent constant-voltage charging scheme, in which the current starts out very high and decreases over the course of the charge cycle [5]. Because of this, a charger with high efficiency across a wide load range meets the need of today's advanced systems.

The goal of this thesis was to improve efficiency performance especially at low load while maintaining the full-load efficiency. The idea presented in this paper is a digitally-controlled system utilizing parallel-connected DC-DC converters. The use of parallel DC-DC converters will allow for the system to enable fewer converters during low-load operation and more converters during full-load, thus keeping the system operating closer to peak efficiency over a wider load range as compared to a single DC-DC converter.

In this paper, first a MATLAB Simulink model is developed to simulate the proposed system and verify general operation. In this model, a digital controller is coded into MATLAB and run during the simulation. Using the Simulink model as a starting point, a hardware implementation of the system is designed, built, and tested. This hardware is based around off-the-shelf DC-DC converters, a microcontroller, and a load-share controller. Firmware is developed to run on the microcontroller and control the system, and software is developed to run on a personal computer to capture data for the user. The results will show that indeed low-load efficiency can be improved with the proposed system, and that with further development could be implemented on any scale desired. 


\section{Chapter 2. Simulation}

\subsection{Simulink Model Development}

Before implementing this thesis in hardware, a Simulink model was developed and studied. In this paper, the approach taken to model the DC-DC simply involves a load versus efficiency lookup table. Instead of modeling the converter directly, a model is developed which uses only the efficiency characteristics of a given DC-DC converter. In this way, the model can be applied to any converter topology and to any number of different converters within a single topology. The converter is modeled as an efficiency lookup table, with inputs of load current and a control signal to enable or disable each converter. The outputs of the converter are input and output power as well as efficiency. Figure 2-1shows this model with inputs and outputs labeled. An accompanying MATLAB file can be modified to update the lookup table values, as well as the output voltage $\left(\mathrm{V}_{\mathrm{o}}\right)$ value.

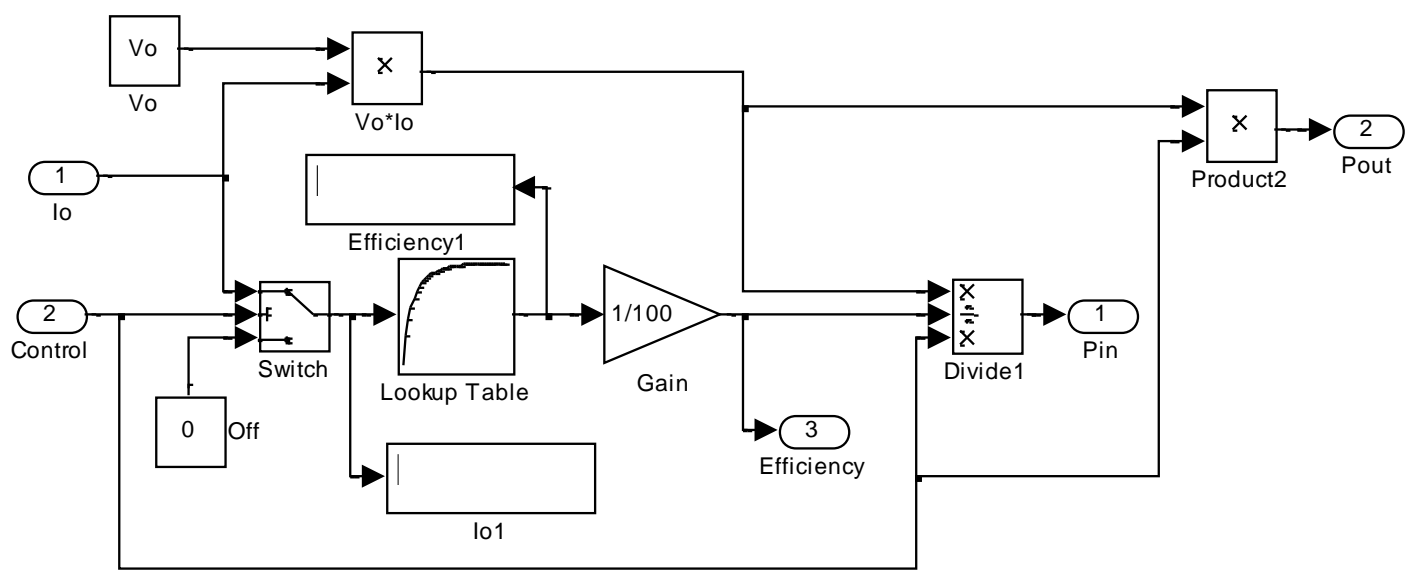

Figure 2-1. DC-DC Converter Efficiency Model

The efficiency model was then integrated into a parallel-connected DC-DC converter setup, as shown in Figure 2-2. In this complete model, total combined output current is divided by the number of active converters (discussed in more detail in the controller section below). This divided output current then feeds into the individual efficiency blocks to determine the efficiency 
of the system. At the output of the efficiency model blocks, the input and output powers are summed and then divided to give a total efficiency for the complete system. Any converter not enabled does not contribute to the total power. A control case efficiency model is also fed by the total output current, and provides a comparison between the paralleled system and an equivalent stand-alone converter. The main controller block shown in Figure 2-2 runs a MATLAB function during runtime which acts as the digital controller of the system. This controller has the ability to enable or disable individual converters. 


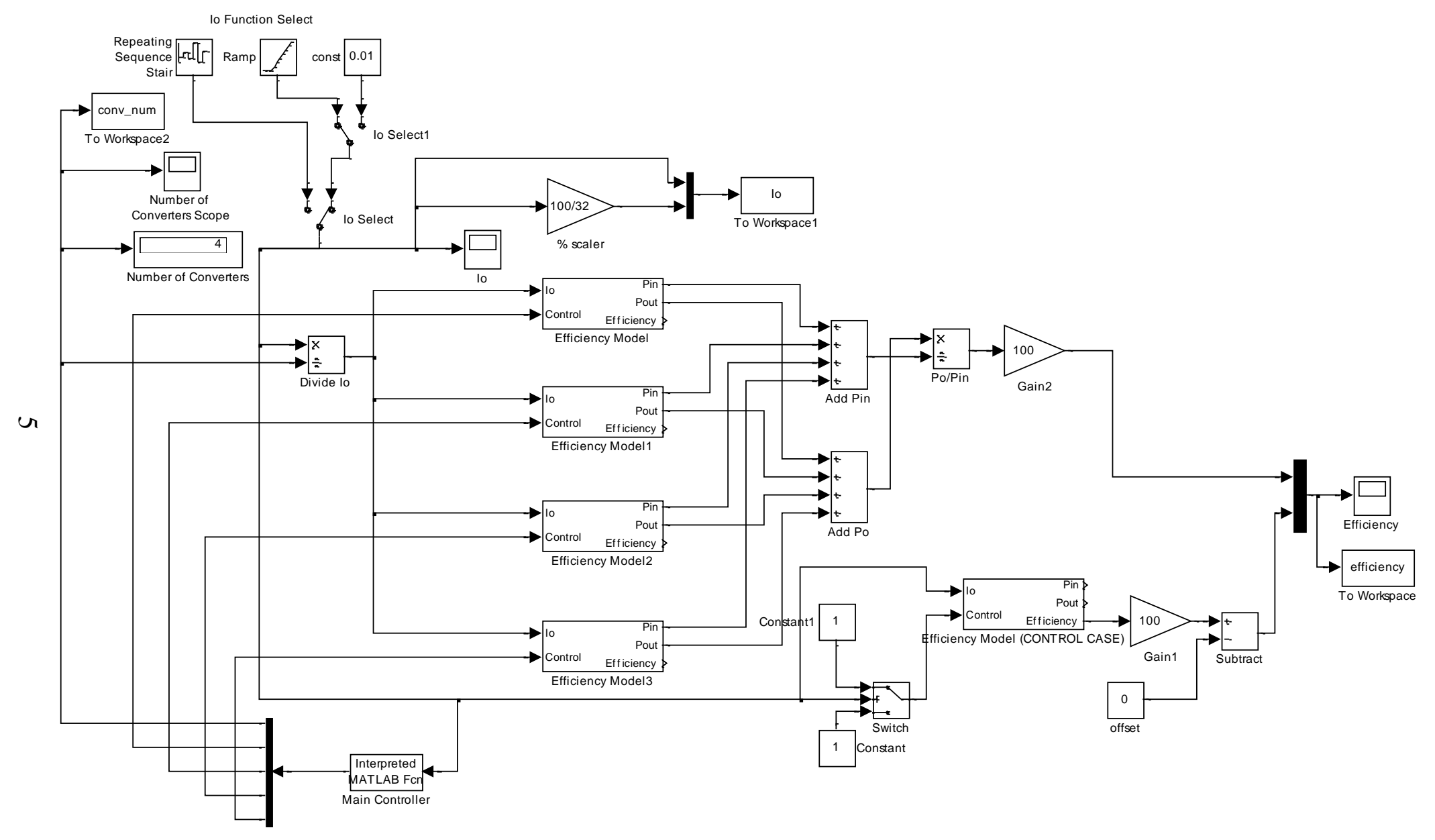

Figure 2-2. Complete Parallel-Connected DC-DC Converter Simulink Model 


\subsection{Controller}

The controller in this model is implemented via a MATLAB function and included in the model shown in Figure 2-2. The present controller is fairly rudimentary, and was written to show the general functionality of the system as a whole. In future work, a much more sophisticated controller can be developed.

To test the validity of the model, example efficiency and load limit values were loaded into the controller. The controller functions by first looking at a table of efficiencies at various loads, and finding the peak efficiency and the corresponding load current. This load current $\left(\mathrm{I}_{\max }\right)$ becomes the current limit at which DC-DC converters are enable or disabled, depending on the total load. As an example, if there are a total of three parallel converters, then the total allowable load current is $3 \mathrm{I}_{\max }$. With three converters, there are three switch points. If the total load current is below $I_{\max }$, then only one converter is enabled. If the current is between $I_{\max }$ and $2 I_{\max }$, then two converters are brought online, and so on.

This control scheme does not employ a master-slave type configuration as discussed in [6] and [7], but rather allows any converter to be enabled individually while the others are disabled. This will allow future development advantages such as thermal stress management. This means that future digital control schemes will look at converter temperature, and depending on the number of parallel converters enabled, will dynamically swap out converters that are running hot (due to long run times or environmental conditions). This adds an added level of control and robustness to the parallel-connected system.

\subsection{Simulation Results and Discussion}

For the simulation, efficiency curves for the paralleled DC-DC converters were taken from the datasheet of the TI PTEA415050N2AD converter (the same converter used in the hardware design). Figure 2-3 shows the curve used. In order to provide a meaningful comparison between 
the paralleled converters and a single stand-alone converter, an off-the-shelf converter was found with a load capacity equal to the total of the paralleled converters. The converter chosen was Murata Power Solutions' UCH-5/30-D48N. As a way to normalize the paralleled converters and the single converter, the peak efficiency of the converters was matched, and the accompanying curve was shifted. This means that since the peak efficiency of the UCH-5/30-D48N was approximately $1 \%$ above that of the PTEA415050N2AD, the curve of the UCH-5/30-D48N was lowered by $1 \%$. Figure 2-4 shows the original and shifted efficiency curves of the UCH-5/30D48N DC-DC converter.

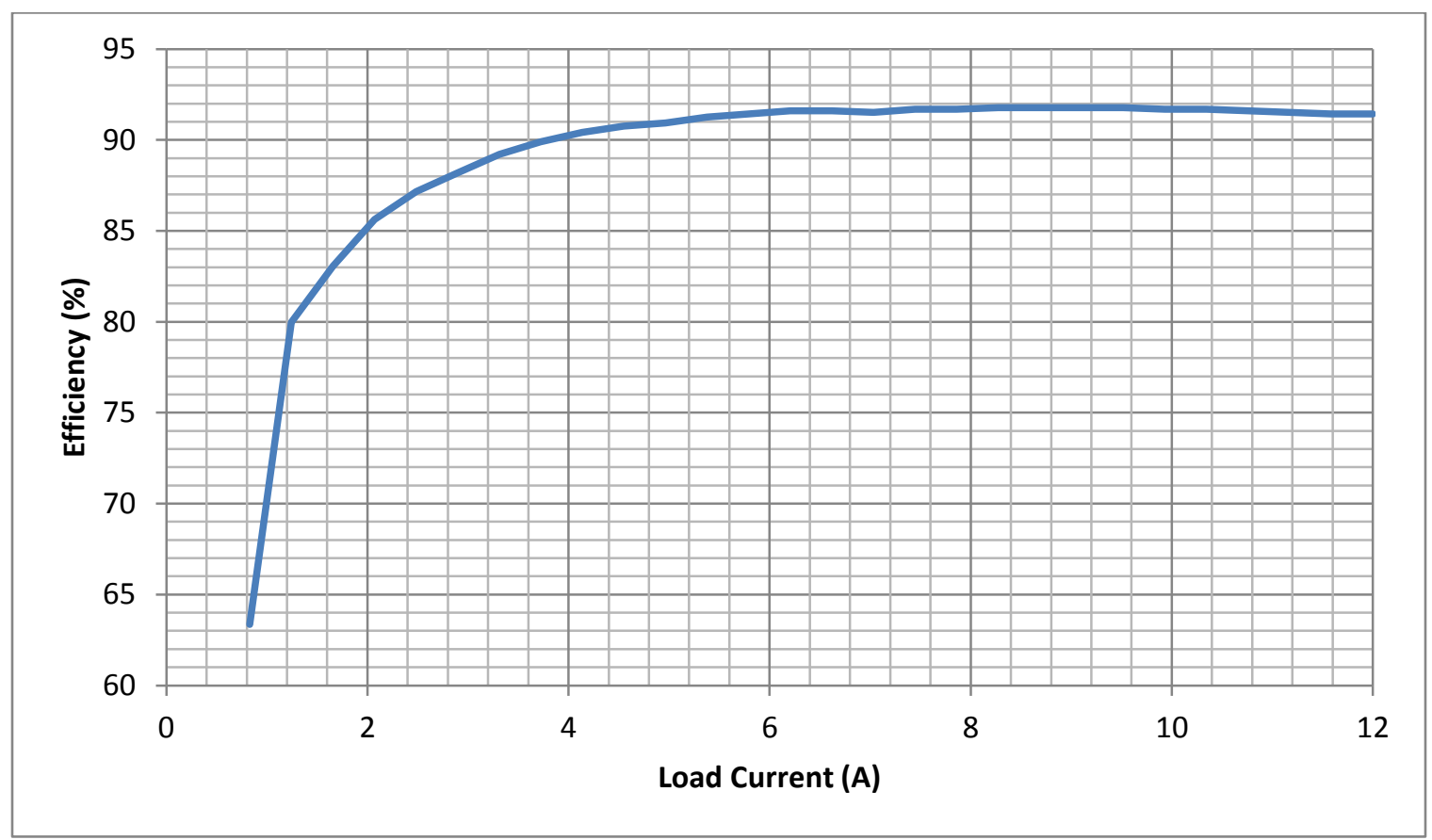

Figure 2-3. Load Current vs. Efficiency of the PTEA415050N2AD DC-DC 


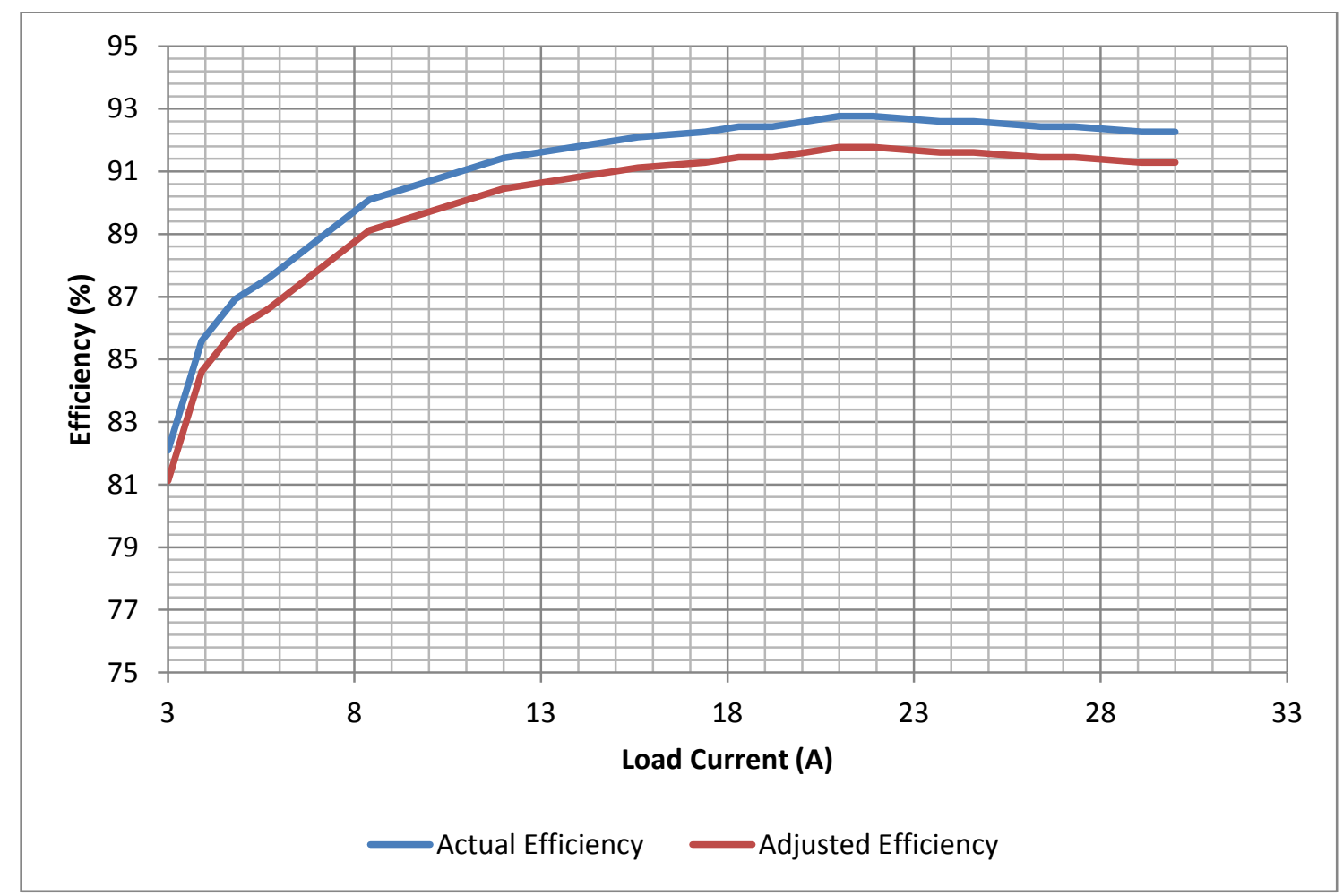

Figure 2-4. Load Current vs. Efficiency of the UCH-5/30-D48N DC-DC

Using the efficiency curves shown above, the simulation was run using an input current ramping from $0 \mathrm{~A}$ to $27 \mathrm{~A}$. Compiling the simulation results produced the efficiency versus percent of total load current graph shown in Figure 2-5. This figure shows the simulation working as expected; from $10 \%$ load to above $70 \%$ load the parallel converter system is operating at a higher efficiency and past that the efficiencies are very close. At $10 \%$ load, the parallel system has approximately an $8 \%$ increase in efficiency. The switch points where consecutive converters are enabled are also evident from Figure 2-5. At $33 \%$ load $(0.33$ x $27 \mathrm{~A}=9 \mathrm{~A})$ the second converter is switched on, indicated by the small step reduction in efficiency. Similarly at $66 \%$ load $(0.66 \times 27 \mathrm{~A}=18 \mathrm{~A})$ the third converter is switched on. To reduce these efficiency steps, the switch current of 9 A was increased to $12 \mathrm{~A}$, and the resulting improved parallel efficiency curve is shown in Figure 2-5. 


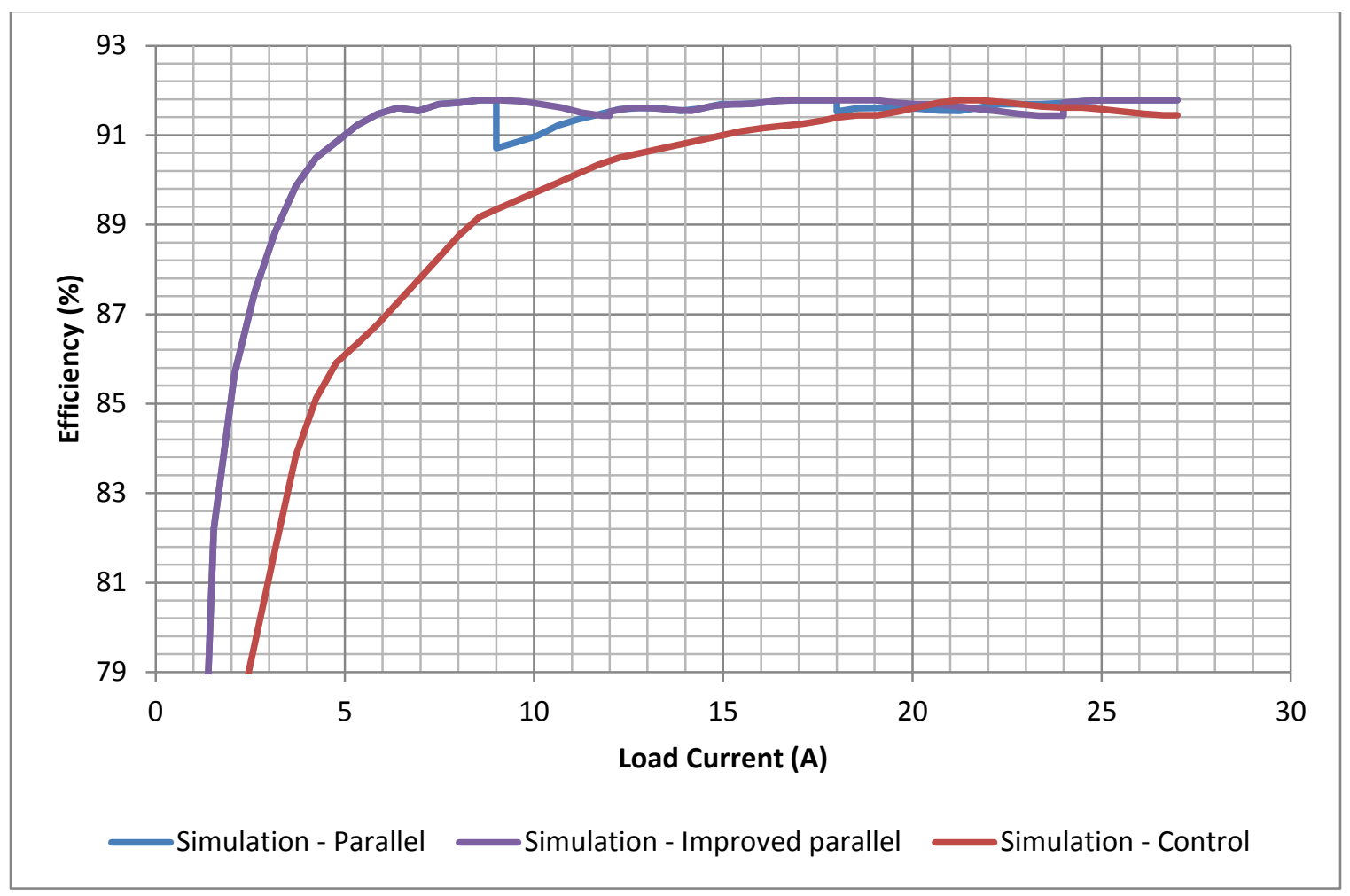

Figure 2-5. Simulation Output Showing Efficiency Curves

These results shown in Figure 2-5 suggest that the proposed parallel-connected DC-DC converters outperform a single converter, especially under low-load conditions. The parallel converter demonstrated better than $88 \%$ efficiency for almost $90 \%$ of its load range, as compared to the single converter which only demonstrates $88 \%$ efficiency or above for $73 \%$ of its load range. From these simulation results it is clear that the improved paralleled system provides demonstrable gains over the single converter, and the remainder of this document will show one way of implementing it in hardware. 


\section{Chapter 3. Design Requirements}

\subsection{Design Goals}

In order to limit the scope of this project to focus on efficiency improvement, certain design requirements were chosen. The hardware for this thesis will be based around three off-the-shelf DC-DC converters, each with a nominal input voltage of $48 \mathrm{VDC}$ and an output voltage of 5

VDC. The allowable maximum current for each DC-DC will be 9 A, allowing for a total of $27 \mathrm{~A}$ for the system. This keeps the total output power of the system ( $27 \mathrm{~A} \times 5 \mathrm{~V}=135 \mathrm{~W})$ below the $150 \mathrm{~W}$ maximum of the available electronic load used for testing. These three DC-DC converters will be integrated into a system controlled by a microcontroller running custom firmware. The firmware will need to know input and output voltage and current for each DC-DC converter in order to calculate efficiency. This information will be provided to the microcontroller via sensors integrated into the system, discussed in more detail below. The main function of the embedded firmware will control the number of active DC-DC converters based on total system load. A loadshare controller will be integrated into the output stage of the system in order to ensure equal currents through the enabled DC-DC converters. This load-share controller will be an integratedcircuit solution with supporting circuitry designed around the system's parameters. The microcontroller and supporting circuitry will be isolated from the power systems, including sensor outputs and control lines.

To accompany the hardware system, a computer-based software application will be developed to allow the user to view system parameters and record data. This software will receive information from the hardware system via serial communications with the microcontroller. This software application will also allow users who are unfamiliar with the hardware a simple means to view and gather data. For this thesis, the software will update at one second intervals. 


\subsection{Design Details}

Figure 3-1 below shows the entire system block diagram, including the controller, power stage, and load-share controller. Each sub-section is contained on its own printed circuit board (PCB). Figure 3-2 shows the power distribution and the $0 \mathrm{~V}$ references within the system. An explanation of each sub-section follows.

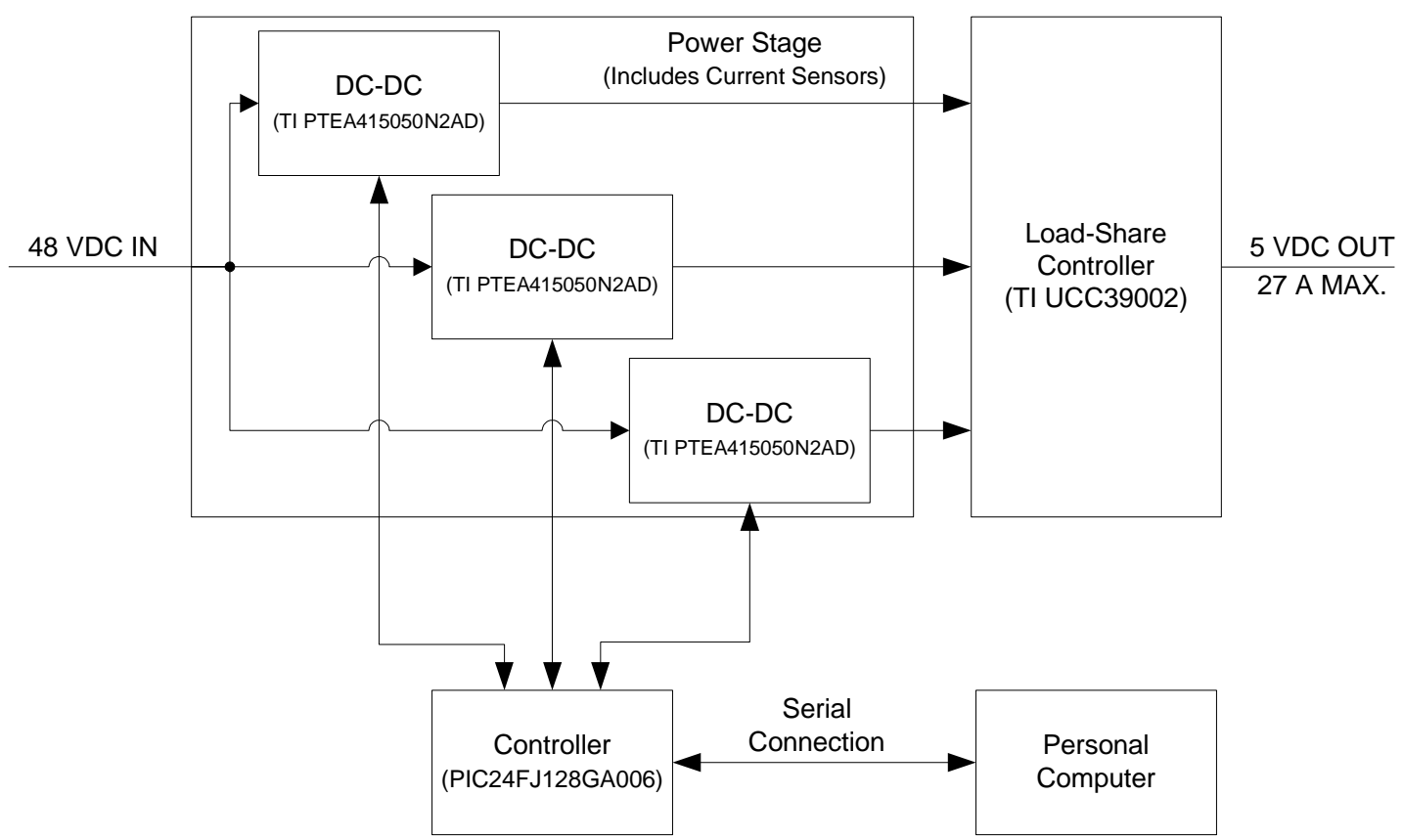

Figure 3-1. System Block Diagram 


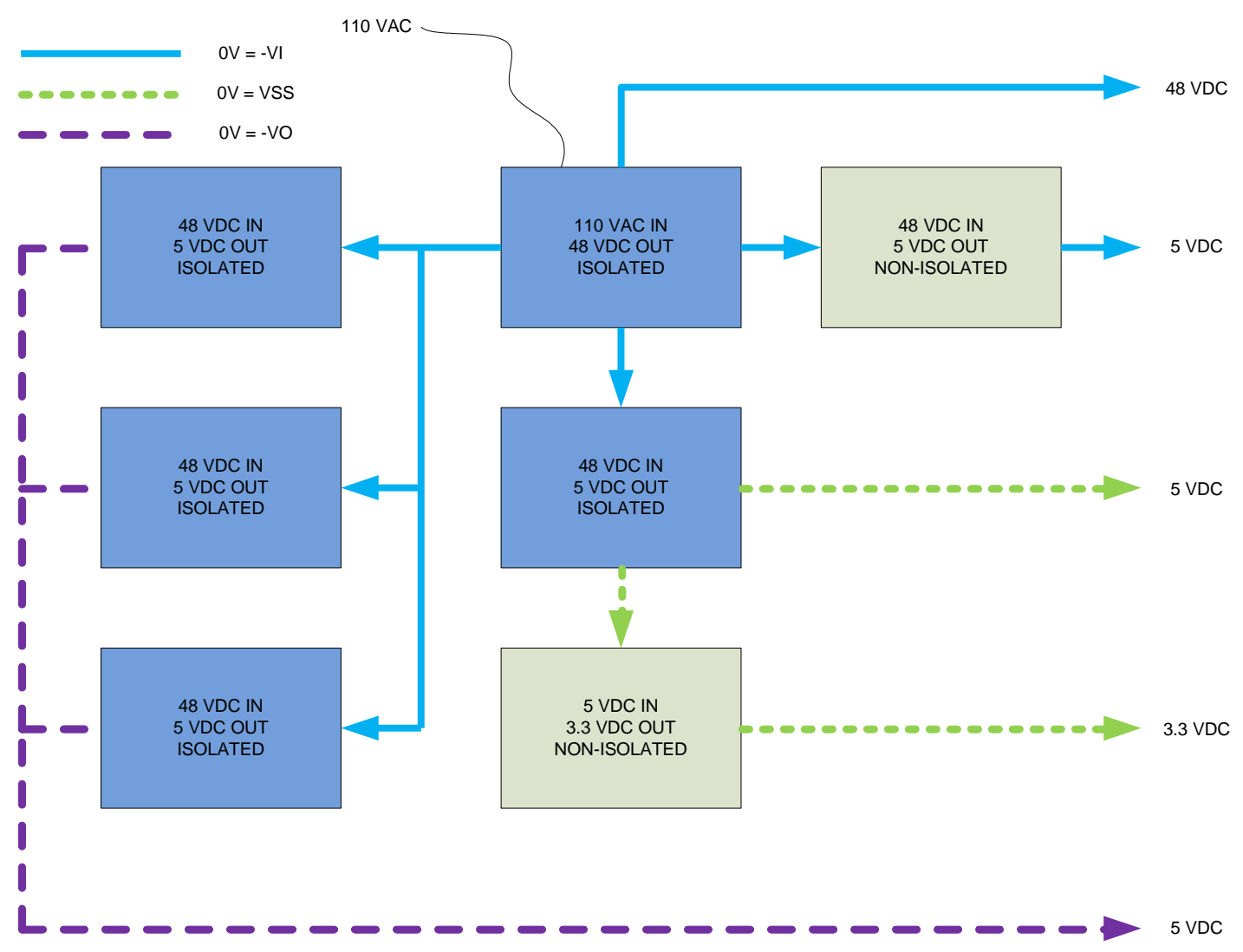

Figure 3-2. Power Distribution and Reference Voltages

\subsubsection{Controller}

The controller is based around a microcontroller made by Microchip, part number PIC24FJ128GA006. This is a 16-bit microcontroller set up to run at a clock speed of $20 \mathrm{MHz}$.

Figure 3-3 below shows the microcontroller and its basic support circuitry required to run. This includes power supply filtering capacitors and the $20 \mathrm{MHz}$ clock crystal. Also shown are the inputs from the current and voltage sensors used to monitor the power stage of the system, as well as some user inputs/outputs, serial communications, and power stage enable lines. 


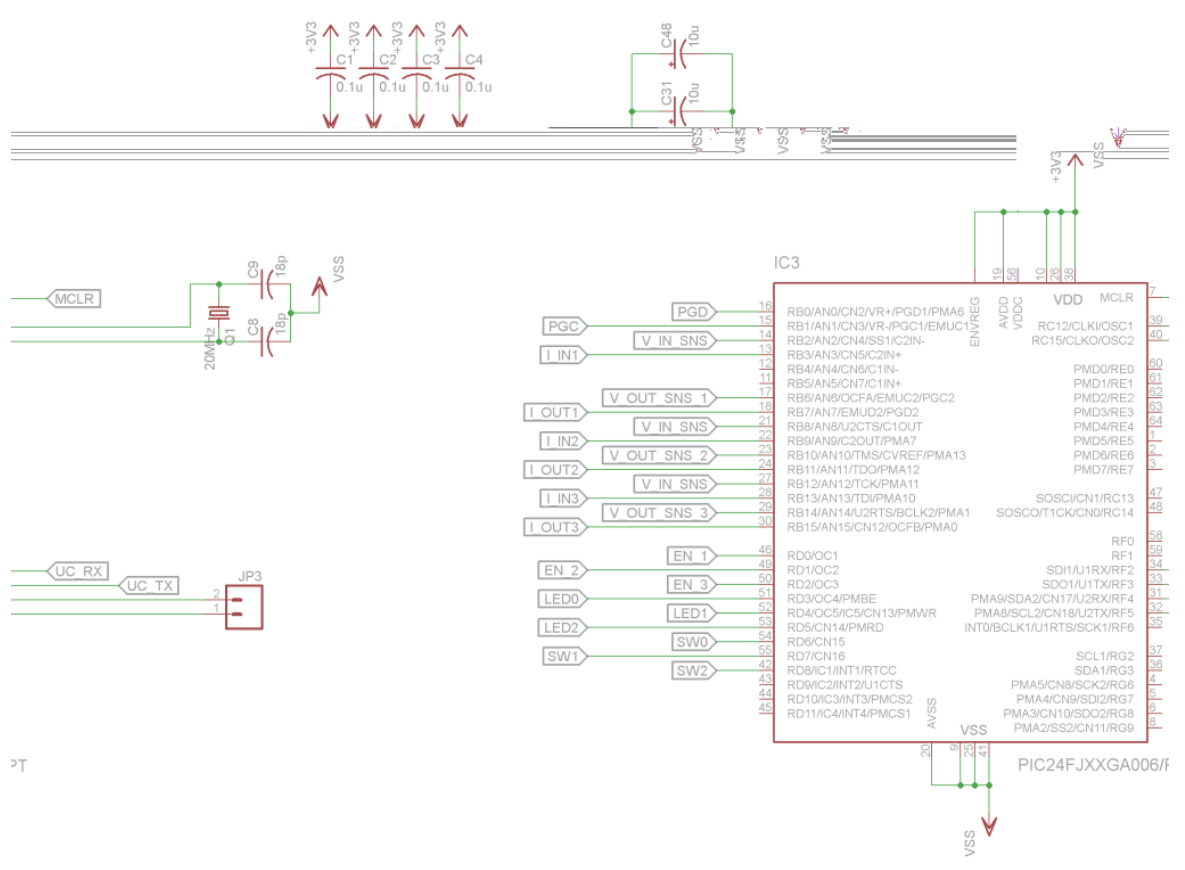

Figure 3-3. Microcontroller and Support Circuitry

The PIC24FJ128GA006 has a 16 channel 10-bit analog-to-digital (A/D) converter built in. This thesis uses twelve of the A/D channels for reading input and output currents and voltages of the three DC-DC converters in the power stage. The A/D is configured to sequentially read the twelve A/D channels and store the data in a buffer (more on this in the software section below). To sense DC-DC voltages, the controller PCB incorporates four isolated voltage sense circuits; one for input voltage and three for each DC-DC output voltage. Figure 3-4 below shows the input voltage sense circuit. The other three circuits are identical except for the input resistor divider resistor R18 is set to a different value. 


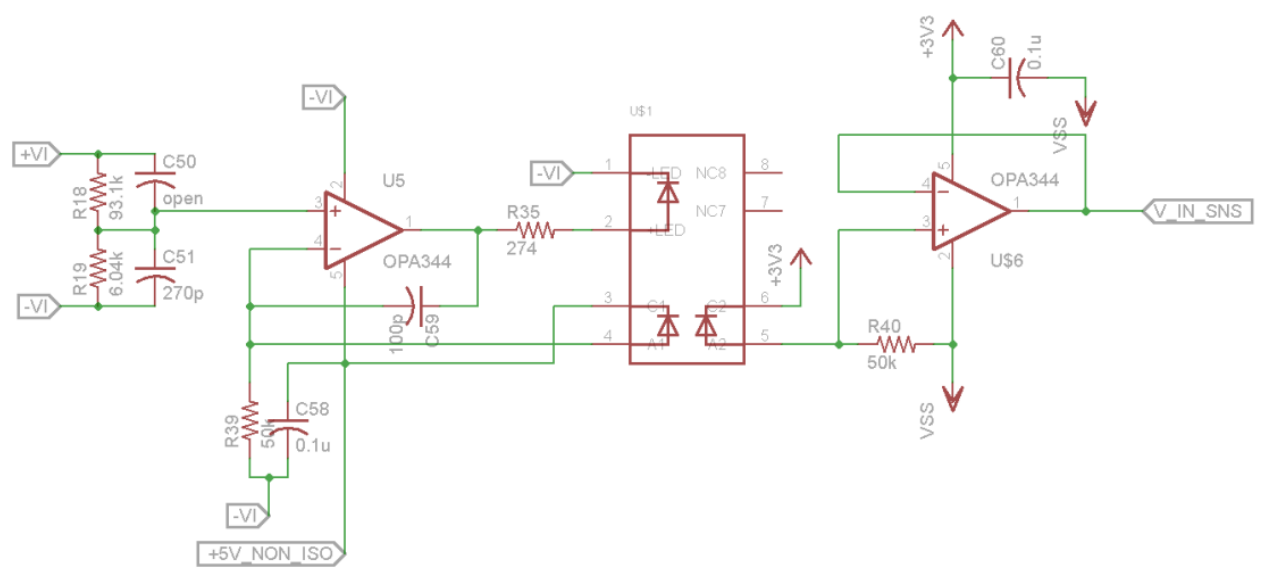

Figure 3-4. Voltage Sense Circuit

To calculate the resistor divider (R18 and R19), input and output voltage swings were determined based on manufacturer datasheets. Since the A/D has a reference voltage of 3.3 VDC, the desired voltage divider output was set to 3 VDC at maximum input and output voltage. This value was set to give maximum A/D range while still allowing for some small voltage overshoot. To reduce power loss through the sense circuit, a current of $500 \mu \mathrm{A}$ through the voltage divider was chosen. Table 3-1 below summarizes the values used for the voltage divider calculations, where $\mathrm{V}_{\mathrm{IN}}$ and $\mathrm{V}_{\text {OUT }}$ represent the input and output voltages of the DC-DC, respectively.

Table 3-1. Resistor Divider Voltage Set Values

\begin{tabular}{cccc}
\hline Parameter & Minimum & Typical & Maximum \\
\hline $\mathrm{V}_{\mathrm{IN}}(\mathrm{V})$ & 46 & 48 & 50 \\
$\mathrm{~V}_{\text {OUT }}(\mathrm{V})$ & 4.85 & 5.00 & 5.15 \\
$\mathrm{~V}_{\text {SET }}(\mathrm{V})$ & N/A & 3 & N/A \\
$\mathrm{I}_{\mathrm{SET}}(\mu \mathrm{A})$ & N/A & 500 & N/A \\
\hline
\end{tabular}


Using the values from Table 3-1, (3-1) shows the total desired resistance. Choosing $\mathrm{R}_{19}=6$ $\mathrm{k} \Omega$ and using (3-2), the desired resistor values are set.

$$
\begin{gathered}
R_{18}+R_{19}=\frac{V_{I N-M A X}}{I_{S E T}}=\frac{50 \mathrm{~V}}{500 \mu \mathrm{A}}=100 \mathrm{k} \Omega \\
R_{18}=\left(R_{18}+R_{19}\right)-R_{19}=100 \mathrm{k} \Omega-6 \mathrm{k} \Omega=94 \mathrm{k} \Omega
\end{gathered}
$$

Choosing standard available resistor values gives $R_{18}=93.1 \mathrm{k} \Omega$ and $R_{19}=6.04 \mathrm{k} \Omega$. These values show the resistor divider for input voltage sensing. Using the same equations and $\mathrm{V}_{\text {OUT }}$ values from Table 3-1 gives resistor values of $R_{18}=4.3 \mathrm{k} \Omega$ and $R_{19}=6 \mathrm{k} \Omega$ for the output voltage sensing. Calculations and more design values for this resistor divider can be found in Table A-2 in Appendix A. Following the resistor divider there is an isolation circuit. This is one of a few means of isolating the control logic/sensing from the main power rails.

The isolation circuit for this voltage sensing is based around a linear optocoupler manufactured by Clare, part number LOC110. As shown in Figure 3-4, the LOC110 requires two op-amps, three resistors, and some filter capacitors for proper operation. The operation of the LOC110 relies on the input voltage being converted into a current which drives an LED, which in turn shines on two phototransistors, one providing feedback and the second driving the output (the LED and phototransistors are integrated in the LOC110). To determine the resistor values, some constants are needed, which can be found in the LOC110 datasheet [8], and are shown in Table 3-2 below. An example design process is provided by Clare in Application Note AN-107 [9]. For both the input and output voltage sense circuits, the LOC110 design will be the same since both resistor dividers can range from $0 \mathrm{~V}$ to $3 \mathrm{~V}$. Since this is the case, the input and output voltage spans for the LOC110 will be set to $0 \mathrm{~V}$ to $3 \mathrm{~V}$. 
Table 3-2. LOC110 Device Characteristics

\begin{tabular}{cccccc}
\hline Parameter & Symbol & Minimum & Typical & Maximum & Units \\
\hline LED Voltage Drop & $\mathrm{V}_{\mathrm{F}}$ & 0.9 & 1.2 & 1.4 & $\mathrm{~V}$ \\
$\mathrm{~K} 1$, Servo Gain $\left(\mathrm{I}_{\mathrm{C} 1} / \mathrm{I}_{\mathrm{F}}\right)$ & $\mathrm{K} 1$ & 0.004 & 0.007 & 0.03 & - \\
K2, Forward Gain $\left(\mathrm{I}_{\mathrm{C} 2} / \mathrm{I}_{\mathrm{F}}\right)$ & $\mathrm{K} 2$ & 0.004 & 0.007 & 0.03 & - \\
Input-Side Supply Voltage & $\mathrm{V}_{\mathrm{CC} 1}$ & 5 & 5 & 5 & $\mathrm{~V}$ \\
\hline
\end{tabular}

Using this information and choosing and LED current $\left(\mathrm{I}_{\mathrm{F}}\right)$ of $15 \mathrm{~mA}, \mathrm{R}_{35}$ from Figure 3-4 is calculated in (3-3) and rounded to $274 \Omega$.

$$
R_{35}=\frac{V_{C C 1 \text { max. }}-V_{\text {Fmin. }}}{I_{F}}=\frac{5 V-0.9 \mathrm{~V}}{15 \mathrm{~mA}}=273.3 \Omega
$$

Next the input $\left(\mathrm{R}_{39}\right)$ and output $\left(\mathrm{R}_{40}\right)$ gain resistors are calculated to be $50 \mathrm{k} \Omega$ as shown in (3-4) and (3-5).

$$
\begin{gathered}
R_{39}=\frac{\text { Input Span Max. }}{K 1_{\text {min. }} * I_{F}}=\frac{3 \mathrm{~V}}{0.004 * 15 \mathrm{~mA}}=50 \mathrm{k} \Omega \\
R_{40}=\frac{\text { output Span Max. }}{K 2_{\text {min. }} * I_{F}}=\frac{3 \mathrm{~V}}{0.004 * 15 \mathrm{~mA}}=50 \mathrm{k} \Omega
\end{gathered}
$$

The above values completely define the necessary external resistors for the LOC110 to operate, in this case as a one-to-one isolator. The output of the op-amp on the output of the LOC110 is now a properly scaled and isolated voltage ready for use by the A/D of the microcontroller.

Also contained on the microcontroller PCB are three power supplies used to power various components on the board. The main input power supply is an off-the-shelf isolated DCDC manufactured by TI, part number PTMA402050N2AD. This converts an input voltage of 48 VDC to 5 VDC. This 5 VDC feeds a linear voltage regulator, part number LM1117 to supply 3.3 VDC to the microcontroller. A second linear voltage regulator, manufactured by Linear Technology, part number LT3012, is fed by the input 48 VDC and drops this down to 5 VDC. The reason for this second 5 VDC regulator is to supply a non-isolated voltage to the input-side op-amps of the optocouplers. 
The remaining components on the control PCB are three momentary push buttons, three LEDs, the In-Circuit Serial Programming (ICSP) circuit for programming the microcontroller, a reset button for the microcontroller, and a USB to Serial converter circuit. The buttons and LEDs provide general purpose input/output (GPIO) for the microcontroller. The complete control board schematic can be found in Appendix A.

\subsubsection{Power Stage}

The power stage of this thesis consists of three DC-DC converters manufactured by TI, part number PTEA415050N2AD. This part was chosen for its input/output voltages and load capacity. Typical input/output characteristics are shown below in Table 3-3. These values meet the design requirements of $48 \mathrm{~V}$ input voltage, $5 \mathrm{~V}$ output voltage, and $9 \mathrm{~A}$ maximum current for each DC-DC in the system. More characteristics of the PTEA415050N2AD can be found in the device datasheet [10]. Figure 3-5 below shows the typical efficiency curves of the PTEA415050N2AD at various input voltages. In this thesis, only an input voltage of $48 \mathrm{~V}$ is considered.

Table 3-3. PTEA415050N2AD Input/Output Characteristics

\begin{tabular}{cccccc}
\hline Parameter & Symbol & Minimum & Typical & Maximum & Units \\
\hline Input Voltage & $\mathrm{V}_{\mathrm{I}}$ & 35 & 48 & 75 & $\mathrm{~V}$ \\
Output Voltage & $\mathrm{V}_{\mathrm{O}}$ & 4.95 & 5 & 5.05 & $\mathrm{~V}$ \\
Output Current & $\mathrm{I}_{\mathrm{O}}$ & 0 & - & 15 & $\mathrm{~A}$ \\
\hline
\end{tabular}




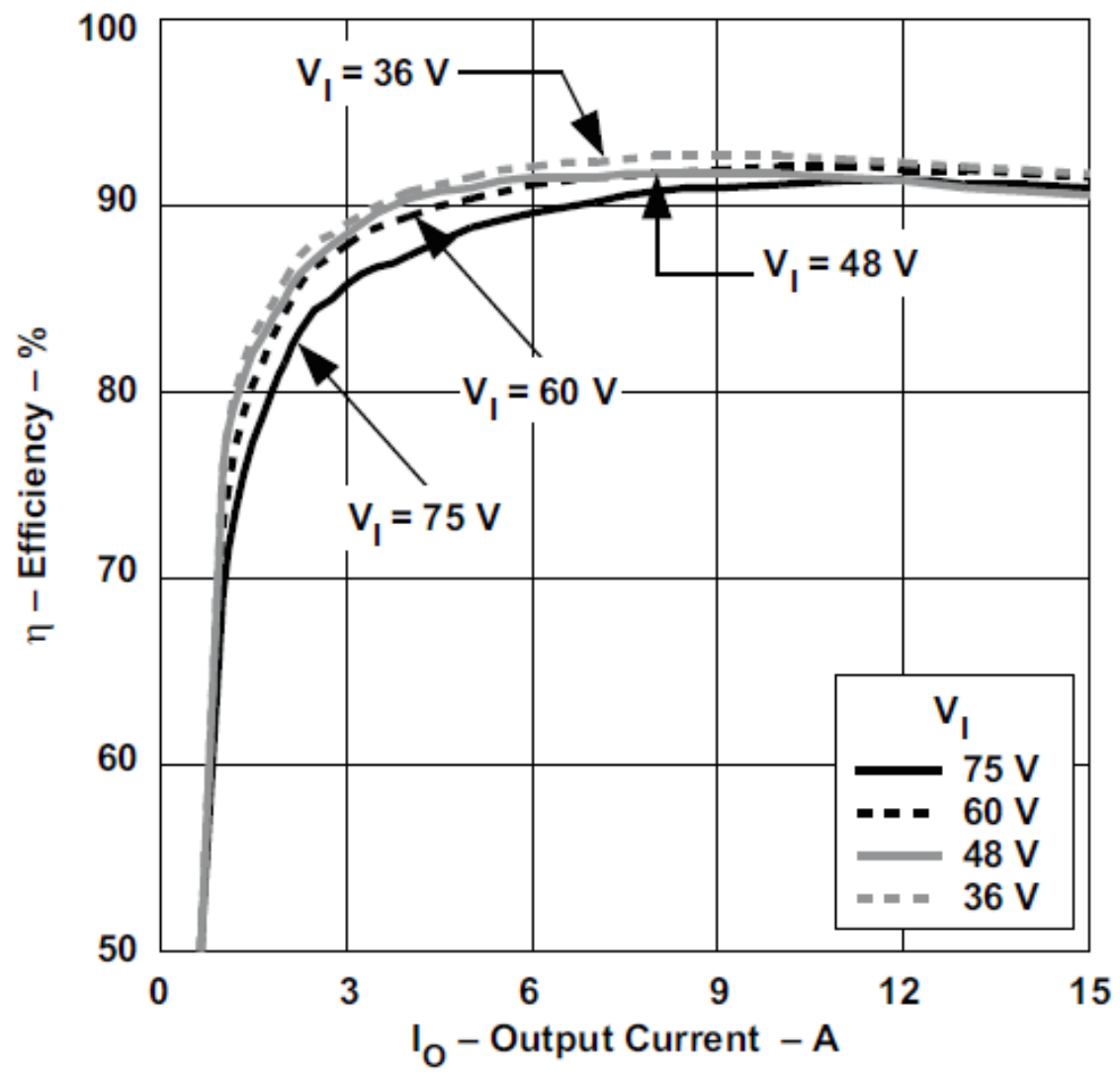

Figure 3-5. Efficiency vs. Output Current for the PTEA415050N2AD (Source: www.ti.com)

The PTEA415050N2AD is a fully isolated DC-DC converter, with the standard application circuit shown in Figure 3-6 below. As shown, pins 1 and 3 are input voltage, pins 8 and 4 are output voltage, 7 and 5 are output voltage remote sense, 6 is output voltage adjust, and 2 is an enable line. In Figure 3-6 the enable line, pin 3, is permanently tied to $-\mathrm{V}_{\mathrm{I}}$ which doesn't allow for dynamic on/off control of the DC-DC. In order for the microcontroller to control whether each DC-DC is on or off, and isolated connection was required between the two. Figure 3-7 shows the circuit used to tie an output of the microcontroller to the enable line of the DC-DC, while maintaining proper isolation. As shown, the microcontroller drives a solid-state relay (SSR) via the "EN_1" line, which in turn controls an n-channel MOSFET. When the MOSFET turns on the DC-DC enable line is pulled to $-\mathrm{V}_{\mathrm{I}}$ and the DC-DC switches on. In Figure 3-6, the remote sense 
pins are tied to the load, but in this thesis these lines are used by the load-share controller, discussed further in the next section of this document.

STANDARD APPLICATION

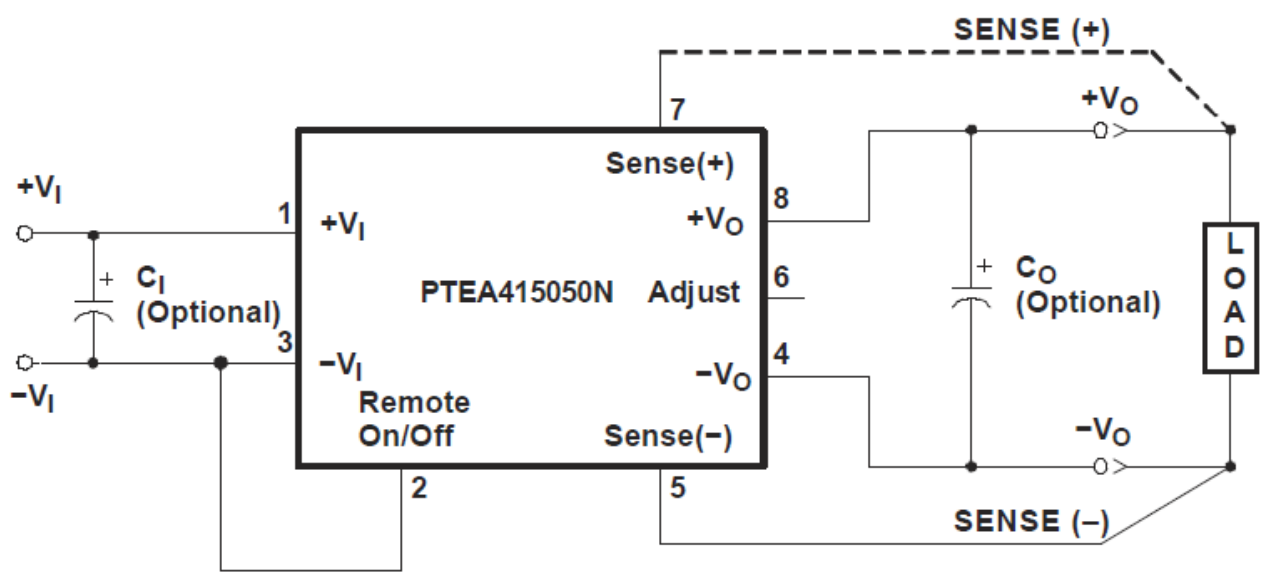

Figure 3-6. Standard Application Circuit for the PTEA415050N2AD (Source: www.ti.com)

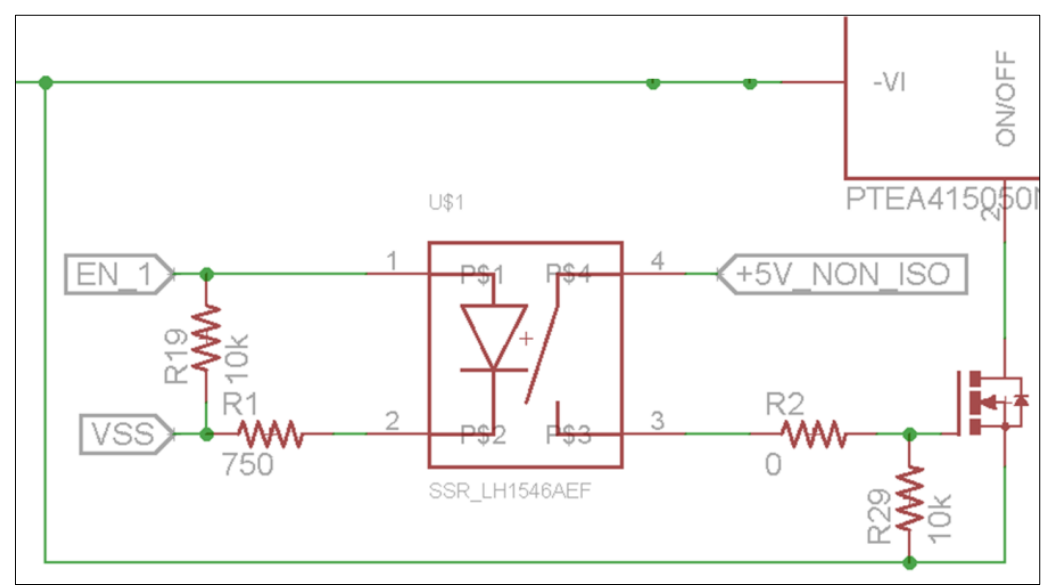

Figure 3-7. Isolated Enable Circuit for PTEA415050N2AD

In order to sense input and output currents of the DC-DC converters (and eventually calculate efficiency), integrated hall effect-based linear current sensors were added to the circuit, one each on the input and output of the DC-DC. Figure 3-8 shows one current sensor and its support circuitry. The sensor is manufactured by Allegro MicroSystems, part number ACS713. The 
ACS713 was chosen for its linearity and low conduction path resistance of $1.2 \mathrm{~m} \Omega$, as shown in the datasheet [11]. The ACS713 converts the measured current into a voltage on the output pin (pin 7) with a scale factor of $0.185 \mathrm{~V} / \mathrm{A}$ and an offset of $0.5 \mathrm{~V}$ at a measured $0 \mathrm{~A}$. Also shown in Figure 3-8 is an op-amp connected to the output of the ACS713 in a non-inverting setup. This provides buffering and scaling between the ACS713 and the microcontroller.

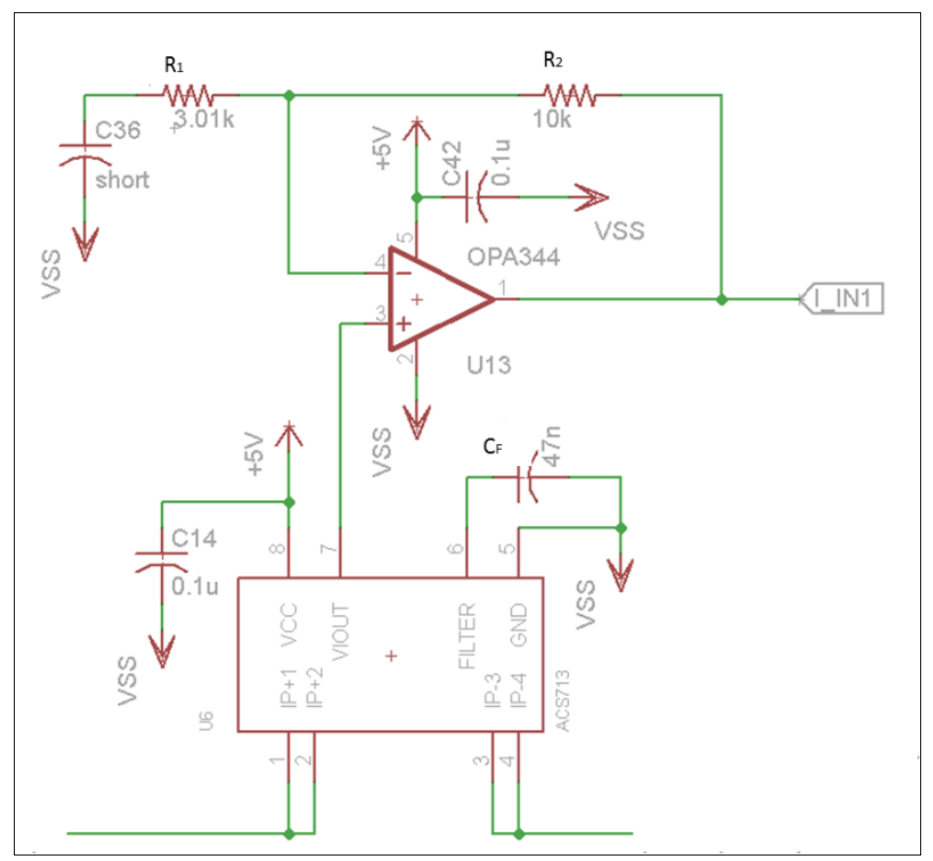

Figure 3-8. ACS713 Current Sense Circuit

In order to obtain maximum resolution of the sensed current, the op-amp was set up to scale the maximum output of the ACS713 up to around $3 \mathrm{~V}$ (similar to the voltage sensing discussed above, this leaves some overhead at the A/D input which can sense up to $3.3 \mathrm{~V}$ ). In order to set the proper gain on the input side, the maximum input current was calculated as shown in (3-6) below.

$$
I_{I N-\max }=\frac{I_{O-\max } * V_{O-t y p}}{V_{I-t y p}}=\frac{9 \mathrm{~A} * 5 \mathrm{~V}}{48 \mathrm{~V}}=0.94 \mathrm{~A}
$$


Adding in a $15 \%$ overhead factor results in $\mathrm{I}_{\mathrm{IN}-\max }=1.1 \mathrm{~A}$. Table 3-4 shows these design values, used to calculate the op-amp gain below.

Table 3-4. ACS713 Design Values

\begin{tabular}{cccc}
\hline Parameter & Symbol & Value & Units \\
\hline ACS713 Gain & ACS $_{\text {GAIN }}$ & 0.185 & $\mathrm{~V} / \mathrm{A}$ \\
ACS713 Offset & ACS $_{\text {OFFSET }}$ & 0.5 & $\mathrm{~V}$ \\
Set Voltage & $\mathrm{V}_{\text {SET }}$ & 3 & $\mathrm{~V}$ \\
Max. Input Current & $\mathrm{I}_{\mathrm{IN}-\max }$ & 1.1 & $\mathrm{~A}$ \\
Max. Output Current & $\mathrm{I}_{\mathrm{OUT}-\max }$ & 9 & $\mathrm{~A}$ \\
Desired Input Gain $=\frac{V_{S E T}}{\left(I_{I N-\max } * A C S_{\text {GAIN }}\right)+A C S_{\text {OFFSET }}}$ \\
$=\frac{3 V}{(1.1 A * 0.185 V / A)+0.5 V}=4.26$ \\
Desired Input Op - Amp Gain $=1+\frac{R_{2}}{R_{1}}=4.26$
\end{tabular}

Setting $R_{2}=10 \mathrm{k} \Omega$ and solving (3-8) gives $R_{1}=3.07 \mathrm{k} \Omega$. In order to increase the $A / D$ resolution and choosing a standard resistor value gives $R_{1}=3.01 \mathrm{k} \Omega$. (3-9) and (3-10) show the calculations for the output circuit.

$$
\begin{gathered}
\text { Desired Output Gain }=\frac{V_{S E T}}{\left(I_{\text {OUT-max }} * A C S_{\text {GAIN }}\right)+A C S_{\text {OFFSET }}} \\
\qquad=\frac{3 \mathrm{~V}}{(9 A * 0.185 \mathrm{~V} / \mathrm{A})+0.5 \mathrm{~V}}=1.39 \\
\text { Desired Output Op }- \text { Amp Gain }=1+\frac{R_{2}}{R_{1}}=1.39
\end{gathered}
$$

In this case setting $R_{1}=10 \mathrm{k} \Omega$ and solving (3-10) gives $R_{2}=3.86 \mathrm{k} \Omega$. In order to increase the $\mathrm{A} / \mathrm{D}$ resolution and choosing a standard resistor values gives $\mathrm{R}_{2}=4.02 \mathrm{k} \Omega$. Using these resistor values, input and output op-amp gain are calculated in (3-11) and (3-12).

$$
\text { Input } O p-\text { Amp Gain }=1+\frac{R_{2}}{R_{1}}=1+\frac{10 k \Omega}{3.01 k \Omega}=4.32
$$




$$
\text { Output Op - Amp Gain }=1+\frac{R_{2}}{R_{1}}=1+\frac{4.02 k \Omega}{10 k \Omega}=1.40
$$

Using this data, the total current to voltage (as seen at the microcontroller) gain can be calculated as shown in (3-13), where I represents either the input or output current through the DC-DC and the respective input or output $O p$-Amp Gain is used.

$$
\text { Gain }=\left[\left(I * A C S_{G A I N}\right)+A C S_{\text {OFFSET }}\right] * O p-\text { Amp Gain }
$$

The op-amp gain values are summarized in Table 3-5 below. Using the values from Table 3-4 and Table 3-5 the current sense resolution can be calculated. One last value, the number of counts in the A/D is needed. Since the A/D uses 10 bits, there are $2^{10}-1=1023$ counts. It is then shown in (3-14) through (3-16) that the input current reading has a resolution of $4.03 \mathrm{~mA} /$ count of the A/D. Similarly, the output current resolution can be calculated and the result of $12.44 \mathrm{~mA} /$ count results.

Table 3-5. Current Sensor Op-Amp Gain Values

$$
\begin{aligned}
& V_{I_{I N-\max }}=\left[\left(I_{I N-\max } A C S_{G A I N}\right)+A_{C S_{O F F S T T}}\right] G A I N_{I N} \\
& =[(1.1 A * 0.185 \mathrm{~V} / \mathrm{A})+0.5 \mathrm{~V}] 4.32=3.04 \mathrm{~V} \\
& V_{I_{I N-\min }}=\left[\left(I_{I N-\min } A C S_{G A I N}\right)+A C S_{O F F S E T}\right] G A I N_{I N} \\
& =\left[\left(0 A * \frac{0.185 \mathrm{~V}}{A}\right)+0.5 \mathrm{~V}\right] 4.32=2.16 \mathrm{~V}
\end{aligned}
$$

\begin{tabular}{cccc}
\hline Parameter & Symbol & Value & Units \\
\hline Input Op-Amp Gain & GAIN $_{\text {IN }}$ & 4.32 & V/V \\
Output Op-Amp Gain & GAIN $_{\text {OUT }}$ & 1.40 & V/V \\
\hline
\end{tabular}

Input Resolution

$$
\begin{aligned}
& =\frac{I_{I N-\max }}{\left[V_{I_{I N-\max }} *\left(\frac{1023 \text { Counts }}{3.3 \mathrm{~V}}\right)\right]-\left[V_{I_{I N-\min }} *\left(\frac{1023 \text { Counts }}{3.3 \mathrm{~V}}\right)\right]} \\
& =\frac{1.1 \mathrm{~A}}{\left(3.04 \mathrm{~V} * \frac{1023 \text { Counts }}{3.3 \mathrm{~V}}\right)-\left(2.16 \mathrm{~V} * \frac{1023 \text { Counts }}{3.3 \mathrm{~V}}\right)} \\
& =4.03 \mathrm{~mA} / \mathrm{count}
\end{aligned}
$$


The final design value for the ACS713 circuit is the filter capacitor, $\mathrm{C}_{\mathrm{F}}$, connected between pins 5 and 6 as shown in Figure 3-8. This filter capacitor determines the bandwidth of the ACS713 as well as the peak-to-peak noise levels. Setting $\mathrm{C}_{\mathrm{F}}=47 \mathrm{nF}$ sets a good trade-off between noise suppression and bandwidth. The ACS713 datasheet shows that with a $\mathrm{C}_{\mathrm{F}}$ of this value, peak-to-peak noise will be approximately $100 \mathrm{~mA}$ and rise time $\left(\mathrm{t}_{\mathrm{r}}\right)($ the time interval between when the device reaches $10 \%$ of its full scale value and when it reaches $90 \%$ of its full scale value) to be $88.2 \mu \mathrm{s}$ [11]. According to the datasheet, the rise time to a step response determines the bandwidth, shown in (3-17). This $\mathrm{C}_{\mathrm{F}}$ is used in both input and output current sense circuits. This completes the power stage details. Figure 3-9 shows one complete sub-section of the power stage. This sub-section is repeated three times to form a complete power stage.

$$
f(-3 d B)=0.35 / t_{r}=0.35 / 88.2 \mu s=3.97 \mathrm{kHz}
$$

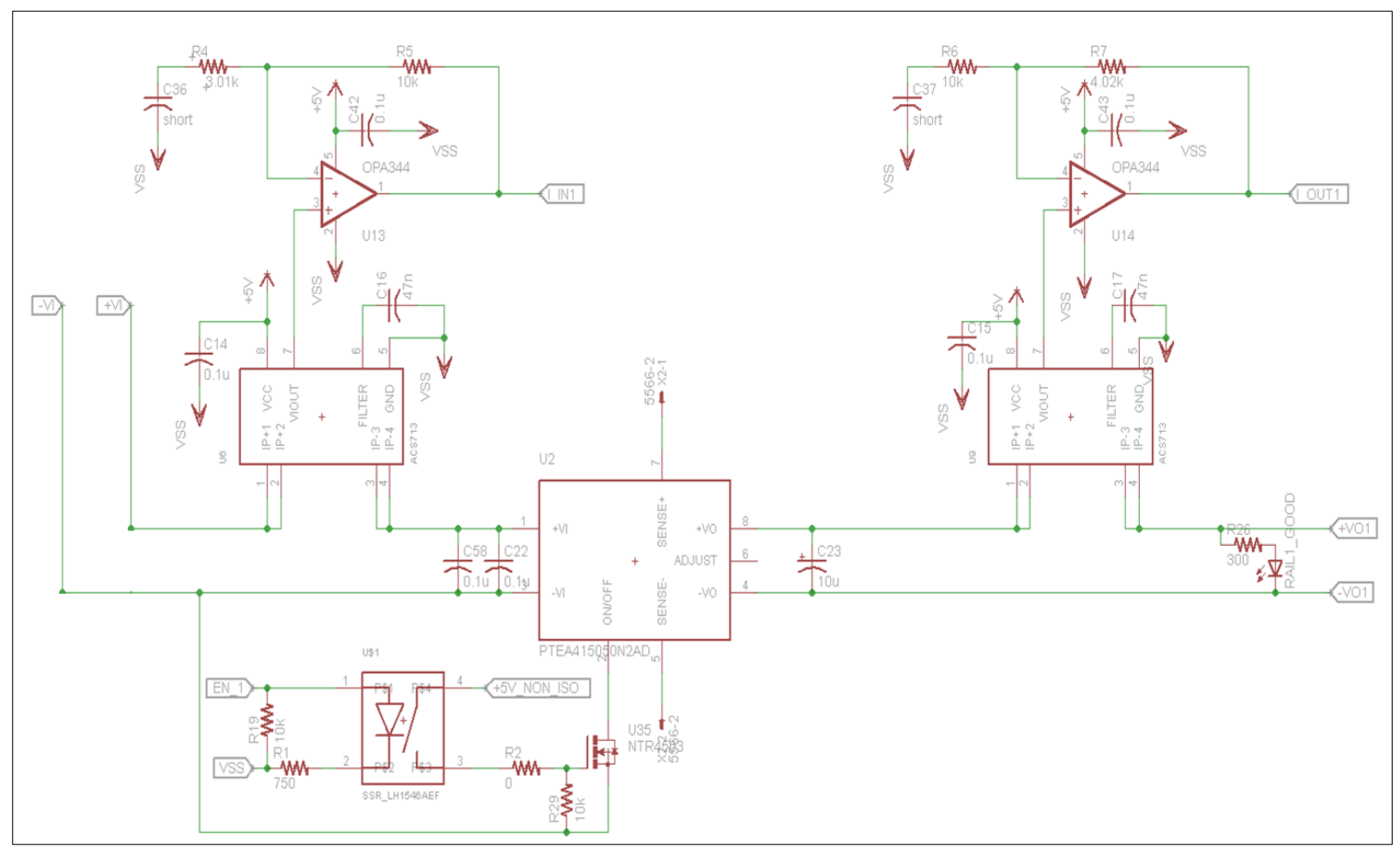

Figure 3-9. Completed Power State Sub-Section 


\subsubsection{Load-Share Controller}

As discussed earlier, paralleled DC-DC converters may not equally share the current being delivered to a load. To remedy this, a load-share controller can be used. The load-share controller in this thesis is based around the TI UCC39002 integrated circuit. The UCC39002 is used to ensure equal currents though multiple paralleled DC-DC converters, as seen in this thesis. It senses the output current of each DC-DC and uses the remote sense lines of the DC-DC to adjust the voltage up or down to fine-tune current. Each DC-DC being paralleled must have a UCC39002 on its output line, and each UCC39002 must communicate (via the Load Share Bus) with the others in order to set equal currents.

In order for the load-share controller to properly work with a given DC-DC, supporting circuitry must be designed to the parameters of the specific DC-DC. One important parameter required is the unity gain crossover frequency of the DC-DC. This is not a parameter given in the datasheet of the PTEA415050N2AD, and so a test was needed to find this value. In the User's Guide of the UCC39002 a method is given to measure unity gain crossover frequency [12]. The setup shown in Figure 3-10 was used, and a Frequency Response Analyzer was used in place of the Network Analyzer shown. 


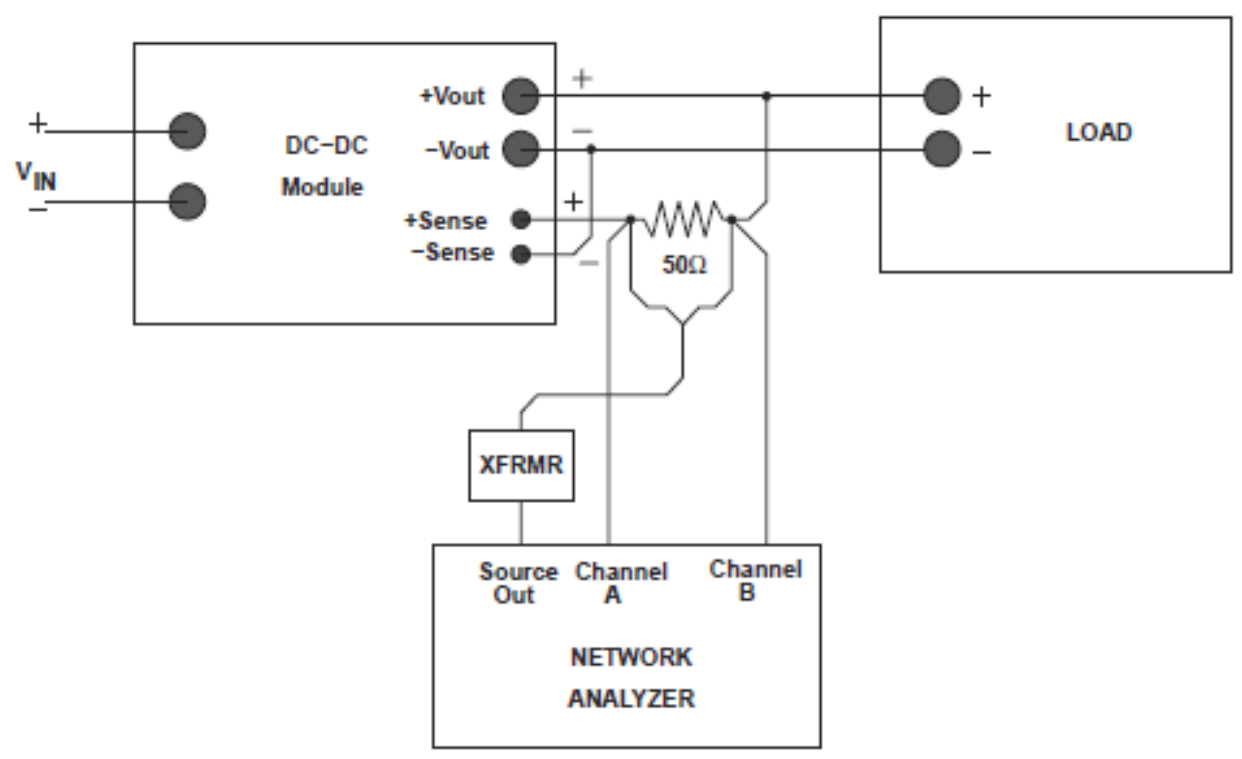

Figure 3-10. Setup to Measure Unity Gain Crossover Frequency of DC-DC (Source: www.ti.com)

The Frequency Response Analyzer used for the test is manufactured by Venable Instruments and is a model 3120 Analyzer. Accompanying instrument software provided a means to capture data during the test. Multiple tests were performed on three PTEA415050N2AD units, each with the test settings shown in Table 3-6. The Bode Plots of each test are shown in Figure 3-11, Figure 3-12, and Figure 3-13, with the unity gain values compiled in Table 3-7. The unity gain crossover frequency used for the design of the load-share controller takes the average of these three values and is $\mathrm{f}_{\mathrm{COMOD}}=21.59 \mathrm{kHz}$.

Table 3-6. Test Parameters Used To Find Unity Gain Crossover Frequency of PTEA415050N2AD

\begin{tabular}{cccc}
\hline Parameter & Symbol & Value & Units \\
\hline Input Voltage & $\mathrm{V}_{\text {IN }}$ & 48.3 & $\mathrm{~V}$ \\
Output Voltage & $\mathrm{V}_{\text {OUT }}$ & 4.87 & $\mathrm{~V}$ \\
Input Current & $\mathrm{I}_{\mathrm{IN}}$ & 1.09 & $\mathrm{~A}$ \\
Output Current & $\mathrm{I}_{\text {OUT }}$ & 8.99 & $\mathrm{~A}$ \\
Start Frequency & $\mathrm{f}_{\text {START }}$ & 10 & $\mathrm{~Hz}$ \\
Stop Frequency & $\mathrm{f}_{\text {STOP }}$ & 240 & $\mathrm{kHz}$ \\
\hline
\end{tabular}




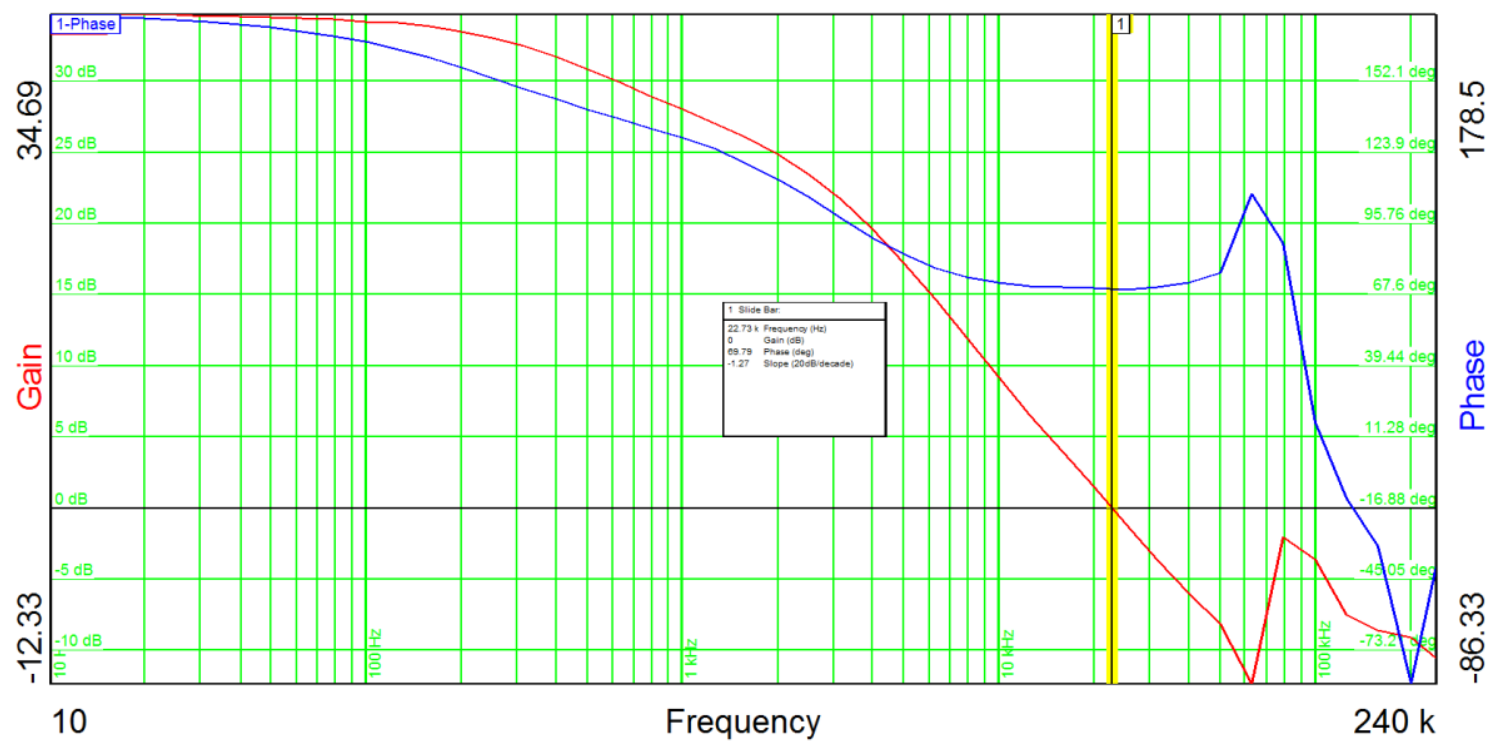

Figure 3-11. Unity Gain Crossover Frequency Bode Plot: Unit 1

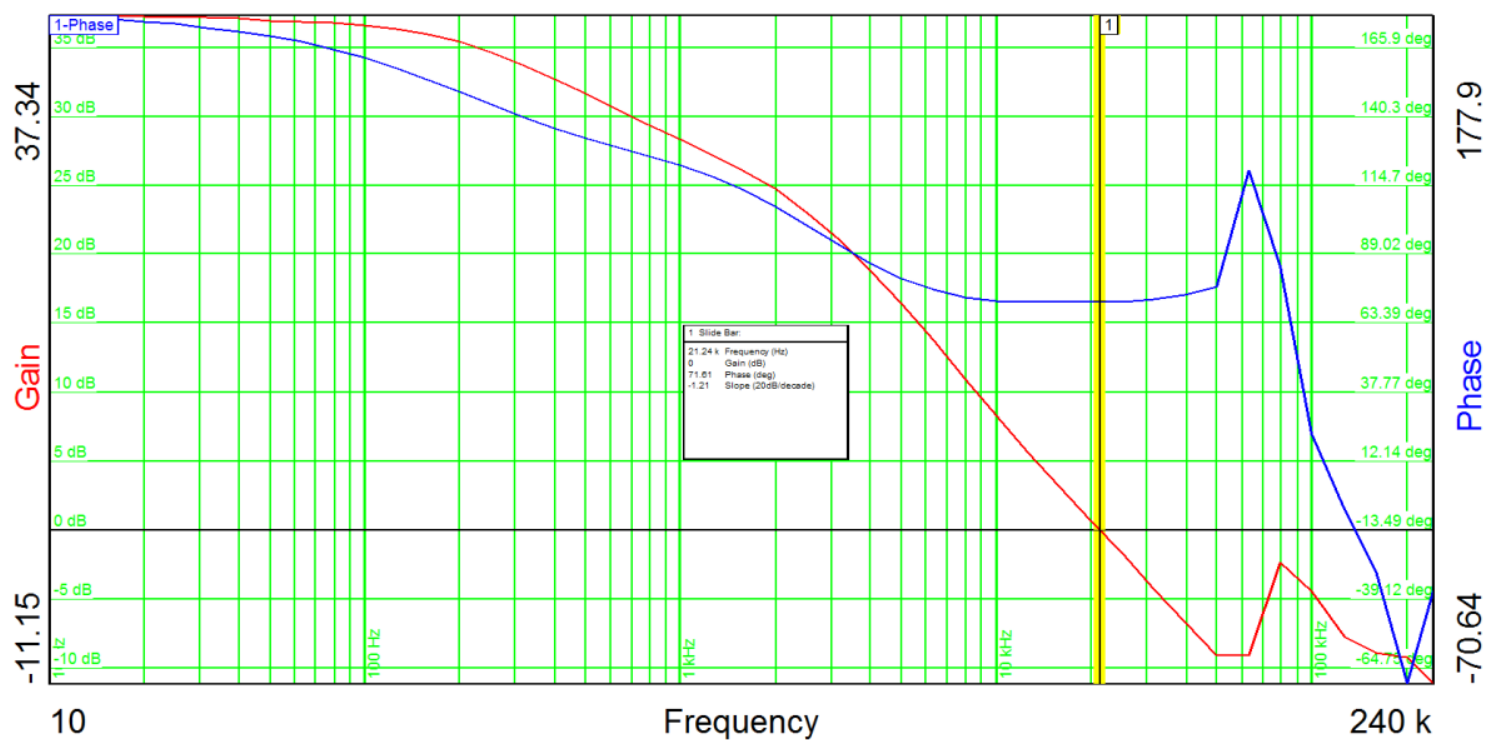

Figure 3-12. Unity Gain Crossover Frequency Bode Plot: Unit 2 


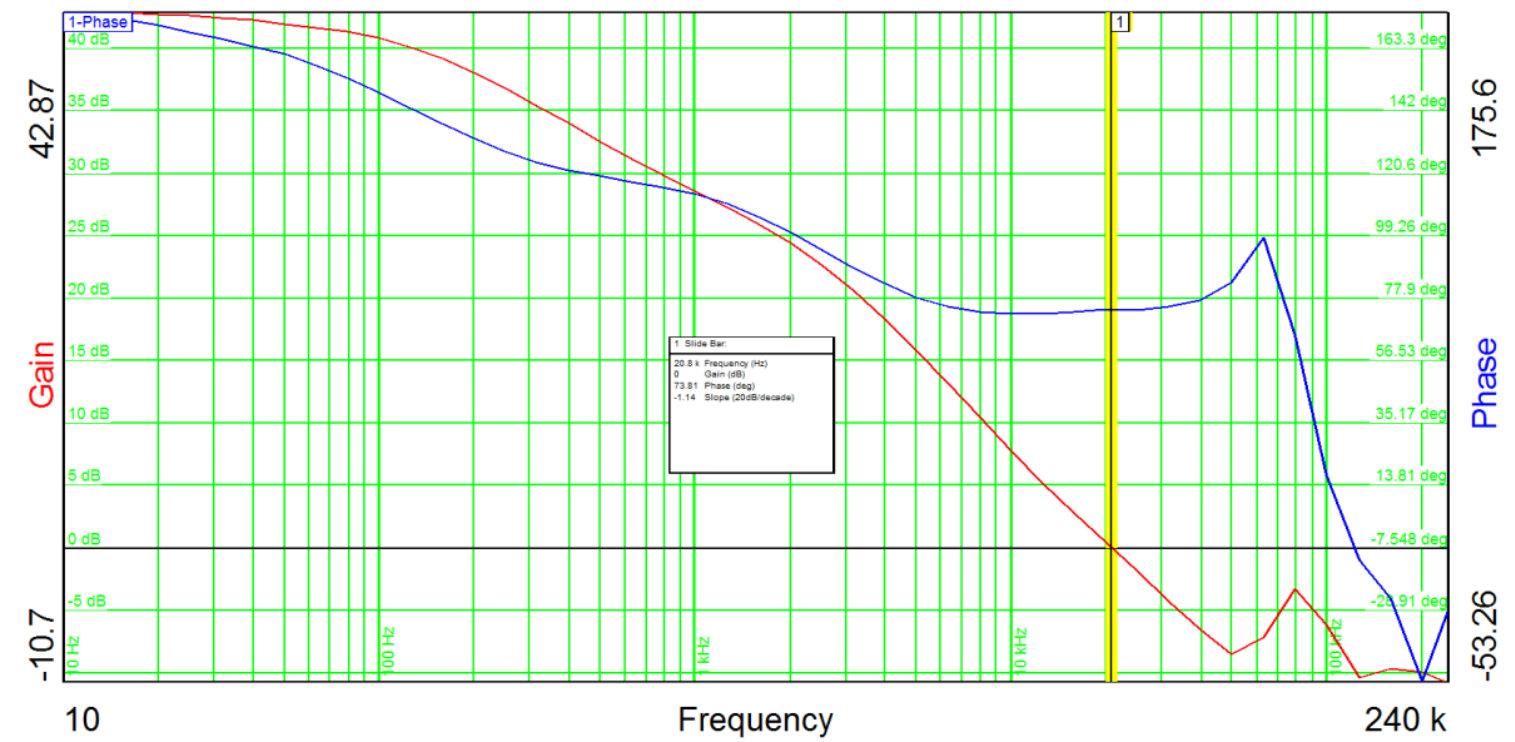

Figure 3-13. Unity Gain Crossover Frequency Bode Plot: Unit 3

Table 3-7. Unity Gain Crossover Frequency Results

\begin{tabular}{cccc}
\hline Parameter & Symbol & Value & Units \\
\hline Unity Gain Crossover Frequency Unit 1 & $\mathrm{f}_{\text {COMOD1 }}$ & 22.73 & $\mathrm{kHz}$ \\
Unity Gain Crossover Frequency Unit 2 & $\mathrm{f}_{\text {COMOD2 }}$ & 21.24 & $\mathrm{kHz}$ \\
Unity Gain Crossover Frequency Unit 3 & $\mathrm{f}_{\text {COMOD3 }}$ & 20.8 & $\mathrm{kHz}$ \\
Unity Gain Crossover Frequency & $\mathrm{f}_{\text {COMOD }}$ & 21.59 & $\mathrm{kHz}$ \\
\hline
\end{tabular}

For this thesis, an evaluation board for the UCC39002 was chosen. The board, part number UCC39002EVM, provides connections for three DC-DC converters and the corresponding space for the UCC39002 support circuitry. Figure 3-14 shows the schematic for the evaluation board. All components labeled "TBD" required design and population; the remaining components were included on the board. The UCC39002EVM User's Guide provides a generic design example, followed here as a groundwork for component values, and available for further design detail [12]. The circuits for all three ICs are the same, so the values were calculated for the first one and copied to the remaining two. 


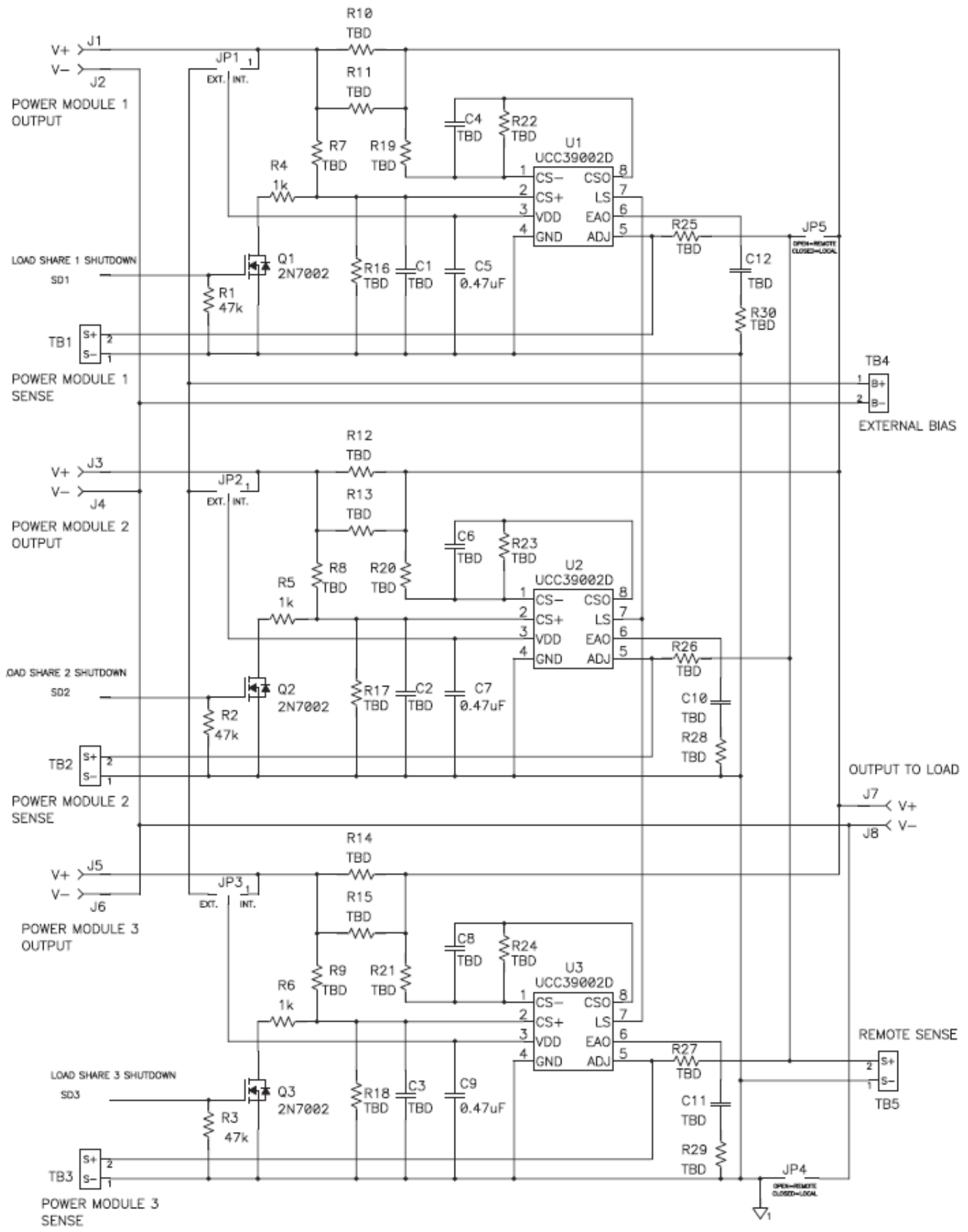

Figure 3-14. UCC39002EVM Load-Share Schematic (Source: www.ti.com) 
A number of constants were required to perform the calculations needed for the circuit design. These constants were gathered from the PTEA415050N2AD datasheet, the UCC39002EVM user's guide, and the design requirements of this thesis. These constants are compiled in Table 3-8, and some are discussed further here. Since the board allows for two $1 \mathrm{~W}$ sense resistors in parallel, TI recommends de-rating the total power and limiting it to $1 \mathrm{~W}$ combined power dissipation, giving $\mathrm{P}_{\mathrm{RSENSE}(\max )}$. The resistors $\mathrm{R}_{\mathrm{CSA} 1}$ and $\mathrm{R}_{\mathrm{CSA} 2}$ set the gain of the current sense amplifier on the UCC39002, and were chosen to set a gain which will be calculated shortly. $\mathrm{f}_{\mathrm{POLE}}$ is an added high frequency pole TI suggests adding to the system for noise roll off, and is set at over twice the unity gain crossover frequency. The gain of the module at the compensation frequency $(22 \mathrm{~Hz}), \mathrm{G}_{\mathrm{MOD}}\left(\mathrm{f}_{\mathrm{ZERO}}\right)$, was read off of Figure 3-11. The remaining values in Table 3-8 appear in the sources discussed above.

Table 3-8. Load-Share Controller Design Constants

\begin{tabular}{cccc}
\hline Parameter & Symbol & Value & Units \\
\hline Nominal Output Voltage of the Modules to be Paralleled & $\mathrm{V}_{\mathrm{OUT}}$ & 5 & $\mathrm{~V}$ \\
Max. Output Current of Each Module to be Paralleled & $\mathrm{I}_{\mathrm{OUT}}(\max )$ & 9 & $\mathrm{~A}$ \\
Max. Output Voltage Adj. Range of Modules to be Paralleled & $\Delta \mathrm{V}_{\mathrm{ADJ}}$ & 0.015 & $\% \mathrm{~V}_{\mathrm{O}}$ \\
Max. Output Voltage Adj. Range of Modules to be Paralleled & $\mathrm{V}_{\mathrm{OUTADJ}(\max )}$ & 0.075 & $\mathrm{~V}$ \\
Number of Modules & $\mathrm{N}$ & 3 & - \\
IC Supply Voltage & $\mathrm{V}_{\mathrm{DD}}$ & 5 & $\mathrm{~V}$ \\
Unity Gain Crossover Frequency of Modules to be Paralleled & $\mathrm{f}_{\mathrm{COMOD}}$ & 21.59 & $\mathrm{kHz}$ \\
Max. Power Dissipation of the Sense Resistor & $\mathrm{P}_{\mathrm{RSENSE}(\max )}$ & 1 & $\mathrm{~W}$ \\
Resistor to Set Current Sense Amplifier Gain & $\mathrm{R}_{\mathrm{CSA} 1}$ & 324000 & $\Omega$ \\
Resistor to Set Current Sense Amplifier Gain & $\mathrm{R}_{\mathrm{CSA} 2}$ & 1000 & $\Omega$ \\
Added High Frequency Pole for Noise Roll Off & $\mathrm{f}_{\mathrm{POLE}}$ & 50 & $\mathrm{kHz}$ \\
Internal Resistance of the LS Pin & $\mathrm{R}_{\mathrm{LS}}$ & 100 & $\mathrm{k} \Omega$ \\
Maximum Current that the Internal Adjust Amplifier can Sink & $\mathrm{I}_{\mathrm{ADJ}(\max )}$ & 6 & $\mathrm{~mA}$ \\
Gain in dB of Module at Compensation Zero Frequency & $\mathrm{G}_{\mathrm{MOD}}\left(\mathrm{f}_{\mathrm{ZERO}}\right)$ & 35.3 & $\mathrm{~dB}$ \\
Transconductance of the Internal Error Amplifier & $\mathrm{G}_{\mathrm{M}}$ & 0.014 & $\mathrm{~S}$ \\
\hline
\end{tabular}

To avoid cumbersome, mostly simple equations, this paper will not show all the equations required for the component design of the UCC39002EVM, as they can all be found in the design example in the user's guide discussed above. Instead only the results are shown in Table 3-9. With these results the UCC39002EVM was fully populated and connected to the 
power stage, completing the hardware design of this thesis. The following section will discuss the hardware design and how the circuits designed in this section were implemented. 
Table 3-9. Load-Share Controller Design Values

\begin{tabular}{|c|c|c|c|}
\hline Parameter & Symbol & Value & Units \\
\hline Maximum value of the current sense resistor for the given allowable power dissipation & $\mathrm{R}_{\mathrm{SENSE}(\max )}$ & 12.3 & $\mathrm{~m} \Omega$ \\
\hline $\begin{array}{l}\text { Actual resistor value based on available standard values } \\
\text { (2512 size, } 1 \mathrm{~W} \text {, room for two in parallel) }\end{array}$ & $\mathrm{R}_{\text {SENSE }}$ & 1 & $\mathrm{~m} \Omega$ \\
\hline Calculated power dissipation of the actual resistor & $\mathrm{P}_{\text {RSENSE }}$ & 81 & $\mathrm{~mW}$ \\
\hline Voltage drop across $R_{\text {SENSE }}$ (should be much less than $\Delta V_{\text {ADJ }}$ ) & $\mathrm{V}_{\text {RSENSE }}$ & & $\mathrm{mV}$ \\
\hline Absolute maximum voltage of the CSO pin & $\mathrm{V}_{\mathrm{CSO}(\max )}$ & 3 & V \\
\hline Absolute maximum allowable current sense gain before saturation & $A_{C S A(\max )}$ & 333.3 & - \\
\hline Actual current sense amplifier gain chosen for this design & $A_{C S A}$ & 324 & - \\
\hline Resultant output voltage of the current sense amplifier and approximate voltage of load share bus & $\mathrm{V}_{\mathrm{CSO}}$ & 2.916 & $\mathrm{~V}$ \\
\hline Calculated capacitor for high frequency pole & $\mathrm{C}_{\mathrm{CSA} \text { (calc) }}$ & 9.8 & $\mathrm{pF}$ \\
\hline Actual value capacitor for high frequency pole & $\mathrm{C}_{\mathrm{CSA}}$ & 10 & $\mathrm{pF}$ \\
\hline Actual high frequency pole & $f_{\mathrm{POLE}}$ & 49.12 & $\mathrm{kHz}$ \\
\hline Increase in master supply current due to $R_{\mathrm{LS}}$ & I MASTERINCREASE(max) & 90 & $\mu \mathrm{A}$ \\
\hline Increase in master power dissipation due to $R_{\mathrm{LS}}$ & $P_{\text {MASTERINCREASE }}$ & 0.45 & $\mathrm{mw}$ \\
\hline Calculated resistor used to set voltage at Sense+ terminal of power module & $\mathrm{R}_{\mathrm{ADJ}(\text { calc) }}$ & 11 & $\Omega$ \\
\hline Actual resistor used to set voltage at Sense+ terminal of power module & $\mathrm{R}_{\mathrm{ADJ}}$ & 11 & $\Omega$ \\
\hline Voltage at $A D J$ pin $\left(V_{A D J} \geq V_{E A O}+1 \mathrm{~V}\right)$ & $\mathrm{V}_{\mathrm{ADJ}}$ & 4.934 & $\mathrm{~V}$ \\
\hline Error amplifier output voltage & $\mathrm{V}_{\text {EAO }}$ & 3 & $\mathrm{~V}$ \\
\hline Minimum to prevent saturating internal adjust amplifier transistor & $\mathrm{R}_{\mathrm{ADJ}(\min )}$ & 8.4 & $\Omega$ \\
\hline $\begin{array}{l}\text { Desired frequency of the error amplifier compensation zero } \\
\text { (placed two decades before the modules crossover frequency) }\end{array}$ & $\mathrm{f}_{\mathrm{ZERO}}$ & 215.9 & $\mathrm{~Hz}$ \\
\hline $\begin{array}{l}\text { Absolute value of gain of power module } \\
\text { (estimated from gain frequency measurement, unit less, not in } \mathrm{dB} \text { ) }\end{array}$ & $\left|G_{M O D}\left(f_{\text {ZERO }}\right)\right|$ & 58.21 & - \\
\hline Load resistance & $\mathrm{R}_{\mathrm{LOAD}}$ & 0.56 & $\Omega$ \\
\hline Voltage gain & $A_{V}$ & 0.0018 & - \\
\hline Adjustment amplifier gain & $A_{A D J}$ & 0.022 & - \\
\hline Calculated capacitor for compensation & $\mathrm{C}_{\mathrm{EAO} \text { (calc) }}$ & 1.32 & $\mathrm{nF}$ \\
\hline Actual capacitor used for compensation & $\mathrm{C}_{\mathrm{EAO}}$ & 120 & $\mathrm{nF}$ \\
\hline Calculated series resistor used in the error amplifier compensation & $\mathrm{R}_{\mathrm{EAO} \text { (calc) }}$ & 6.143 & $k \Omega$ \\
\hline Actual series resistor used in the error amplifier compensation & $\mathrm{R}_{\mathrm{EAO}}$ & 6.19 & $\mathrm{k} \Omega$ \\
\hline
\end{tabular}




\section{Chapter 4. Hardware Design}

This section will describe the physical implementation of the schematic discussed in the Design Details section of this document. As already discussed, the design was broken up into a controller $\mathrm{PCB}$, a power $\mathrm{PCB}$, and a load-share controller $\mathrm{PCB}$, with wires interconnecting everything. An original design combined the controller and power sections and was not fully isolated, and this proved to be a faulty approach. Noise on the ground caused unwanted results, and thus the newer design discussed here was implemented. The following sections discuss specific details of each board.

\subsection{Controller PCB}

The controller PCB was designed using the EAGLE suite of software. Common-practice rules for PCB design were followed, including minimum trace spacing and trace width. The PCB was designed as a two-layer board using 1-ounce copper and an FR-4 substrate. A top power $(+3 \mathrm{~V} 3)$ plane and a bottom ground (VSS) plane were added to improve noise suppression on the PCB. Figure 4-1 and Figure 4-2 show the top and bottom copper layers respectively, while Figure 4-3 and Figure 4-4 show the top and bottom silkscreen layers respectively. The components on the board are grouped in functional sections as drawn on the schematic. The PCB interfaces with the rest of the system via a 24-pin connector and wire harness. A mini-USB port is available for serial connection to a host computer, and there are three general-purpose buttons and LEDs. Test points were added for ease of testing and diagnosis, and a reset provides a means to reboot the system. Two LEDs show the status of the $5 \mathrm{~V}$ and $3.3 \mathrm{~V}$ power rails, and there is a 5-pin header for programming the microcontroller. Finally, there is a jumper, which if removed cuts power to the PCB disabling all functionality. All these features are shown in Figure 4-5. 


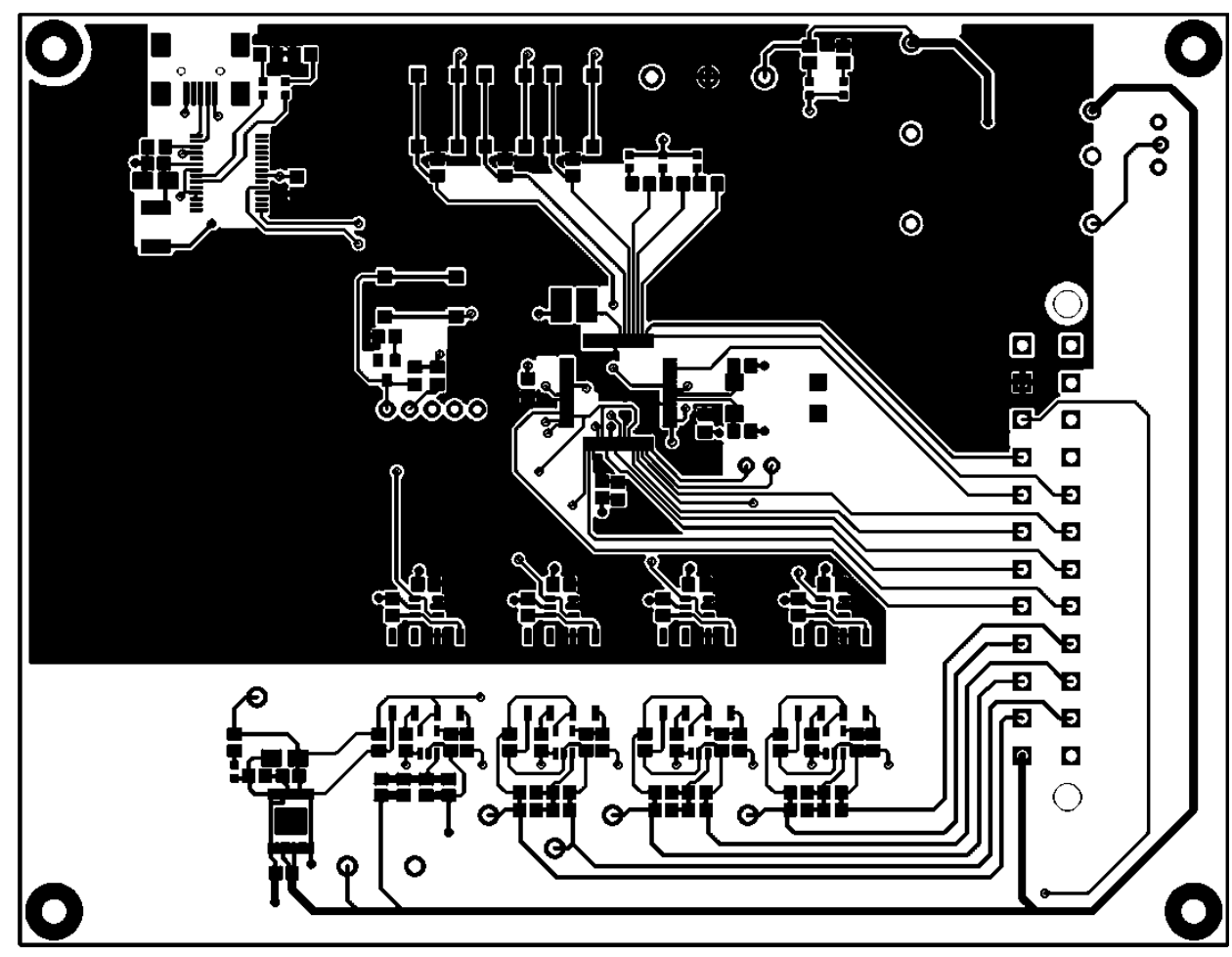

Figure 4-1. Controller PCB Top Copper

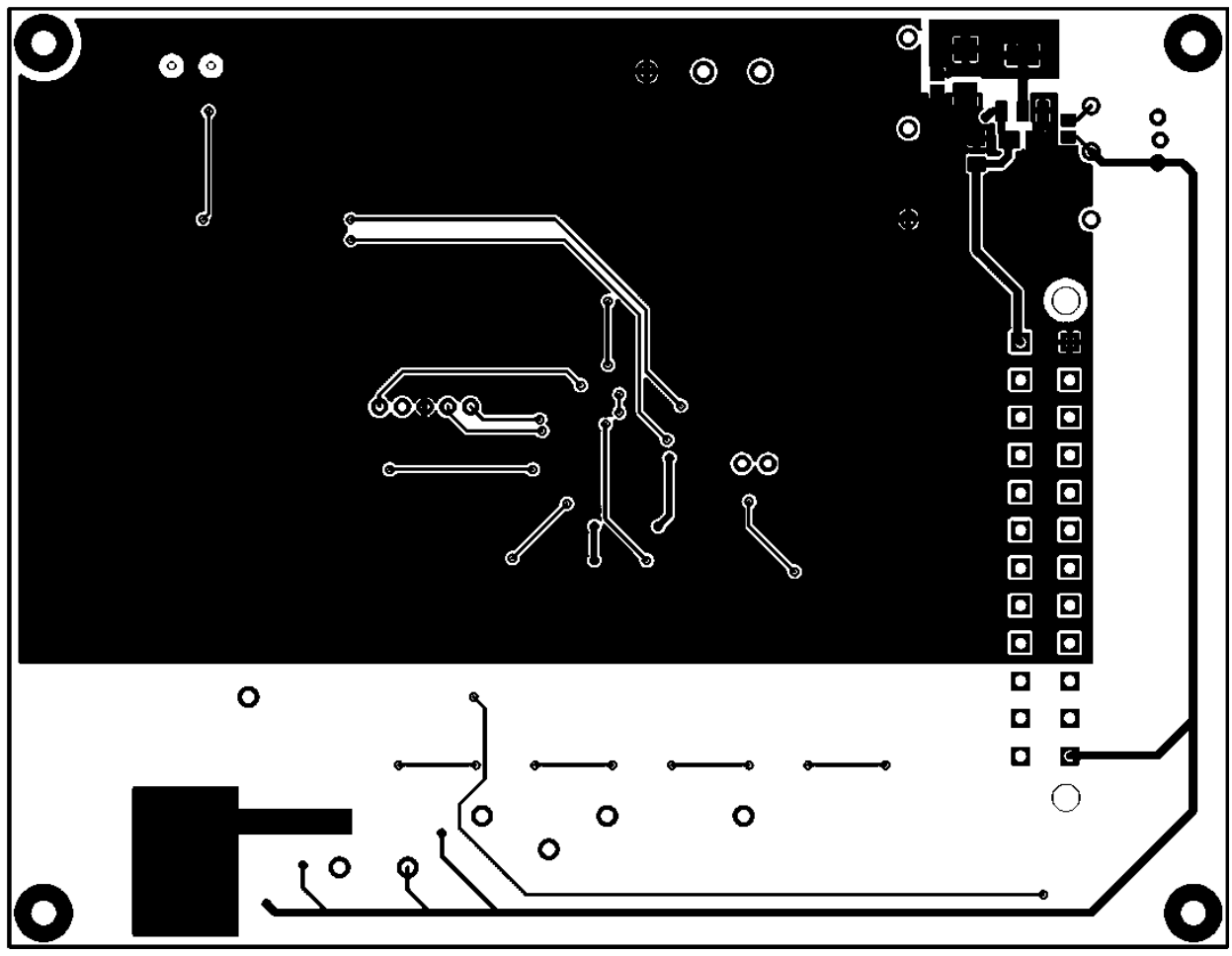

Figure 4-2. Controller PCB Bottom Copper 


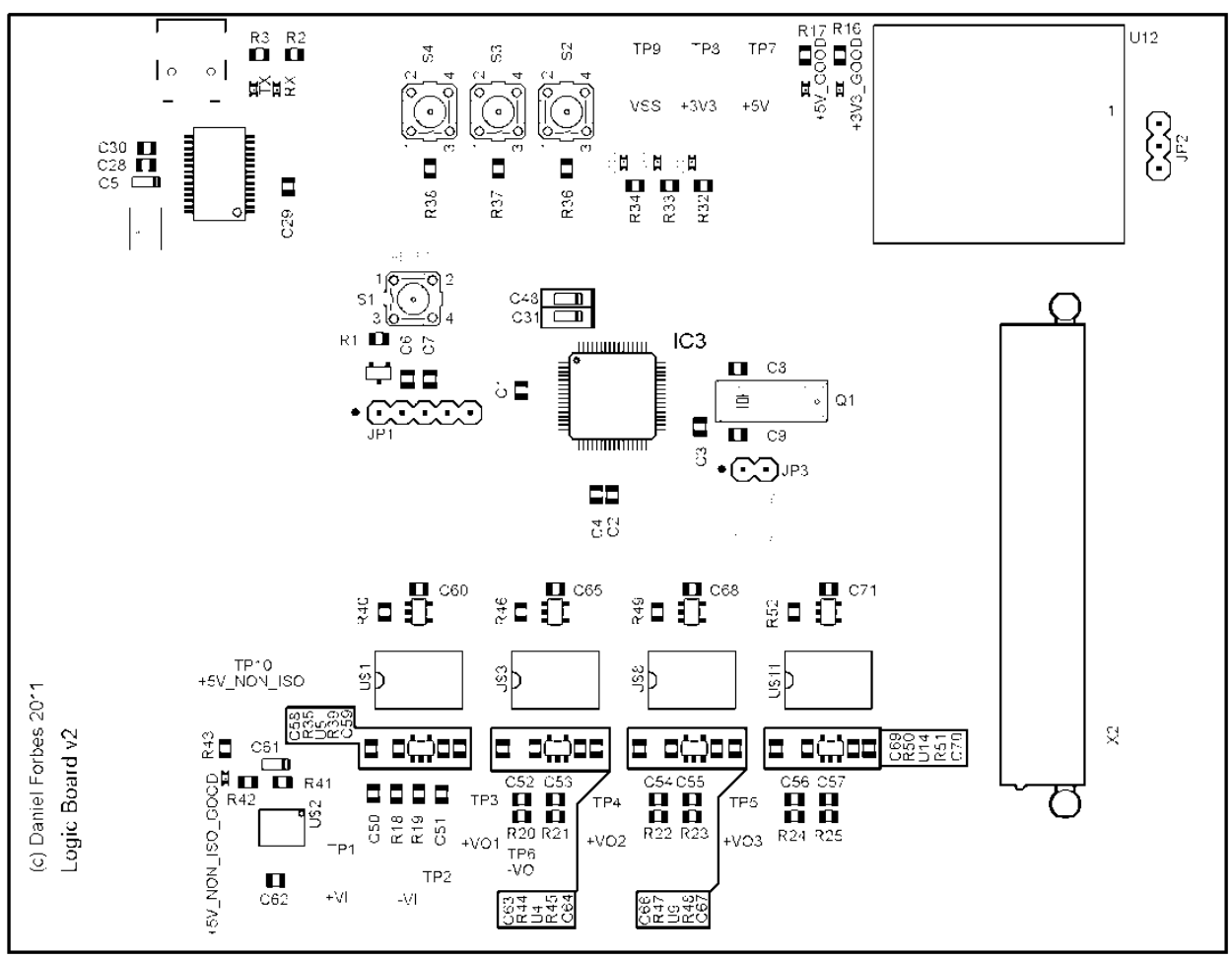

Figure 4-3. Controller PCB Top Silkscreen

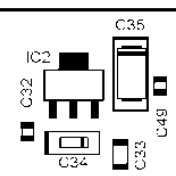

Figure 4-4. Controller PCB Bottom Silkscreen (Mirrored) 


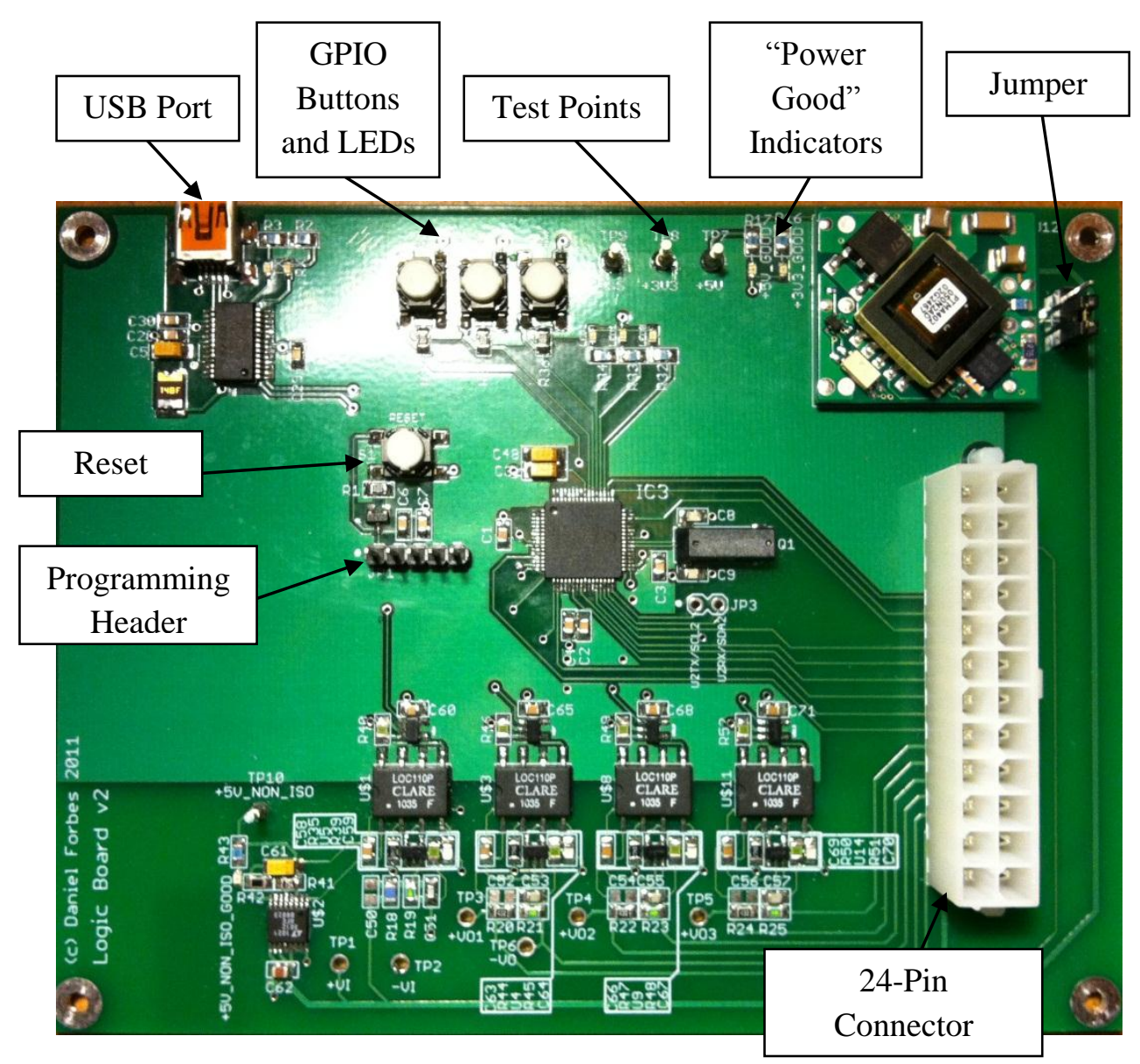

Figure 4-5. Control PCB Photo with Features Labeled

\subsection{Power Stage PCB}

The Power Stage PCB was designed using the EAGLE suite of software. Common-practice rules for PCB design were followed, including minimum trace spacing and trace width. The PCB was designed as a two-layer board using 1-ounce copper and an FR-4 substrate. Special care was taken sizing the input and output power traces going to and from the DC-DC converters, since they are capable of carrying 9 A each. An online trace width calculator using IPC-2221 standards was used for properly sizing the trace width [13]. As an example, for sizing the DC-DC output traces the parameters in Table 4-1 were input into the online calculator, giving the trace width shown in Table 4-1. In the PCB design, a trace width of over $9.5 \mathrm{~mm}$ on both the top and bottom layers was used, giving an effective trace width of $19 \mathrm{~mm}$, well above the recommended width. 
Table 4-1. Example Trace Width Calculation

\begin{tabular}{ccc}
\hline Parameter & Value & Units \\
\hline Current & 10 & $\mathrm{~A}$ \\
Copper Thickness & 1 & $\mathrm{oz}^{\circ} \mathrm{ft}^{2}$ \\
Temperature Rise & 10 & ${ }^{\circ} \mathrm{C}$ \\
Ambient Temperature & 25 & ${ }^{\circ} \mathrm{C}$ \\
Trace Length & 2.54 & $\mathrm{~mm}$ \\
Required Trace Width (External Layer) & 7.19 & $\mathrm{~mm}$ \\
\hline
\end{tabular}

The power PCB connects to the controller PCB via a 24-pin connector and wire harness. Figure 4-6 and Figure 4-7 show the top and bottom copper layers of the PCB respectively, and Figure 4-8 and Figure 4-9 show the top and bottom silkscreen layers of the PCB, respectively. Bolt holes were added for a $48 \mathrm{~V}$ supply hook-up, and also for connection to the load-share controller PCB. Three 2-pin connectors also allow for the load-share controller to connect to the remote sense lines of the three DC-DC converters. Test points provide a means to measure different signals on the board during testing and debugging. Figure 4-10 shows a photograph of the top of the power PCB, labeled with the features just discussed. 


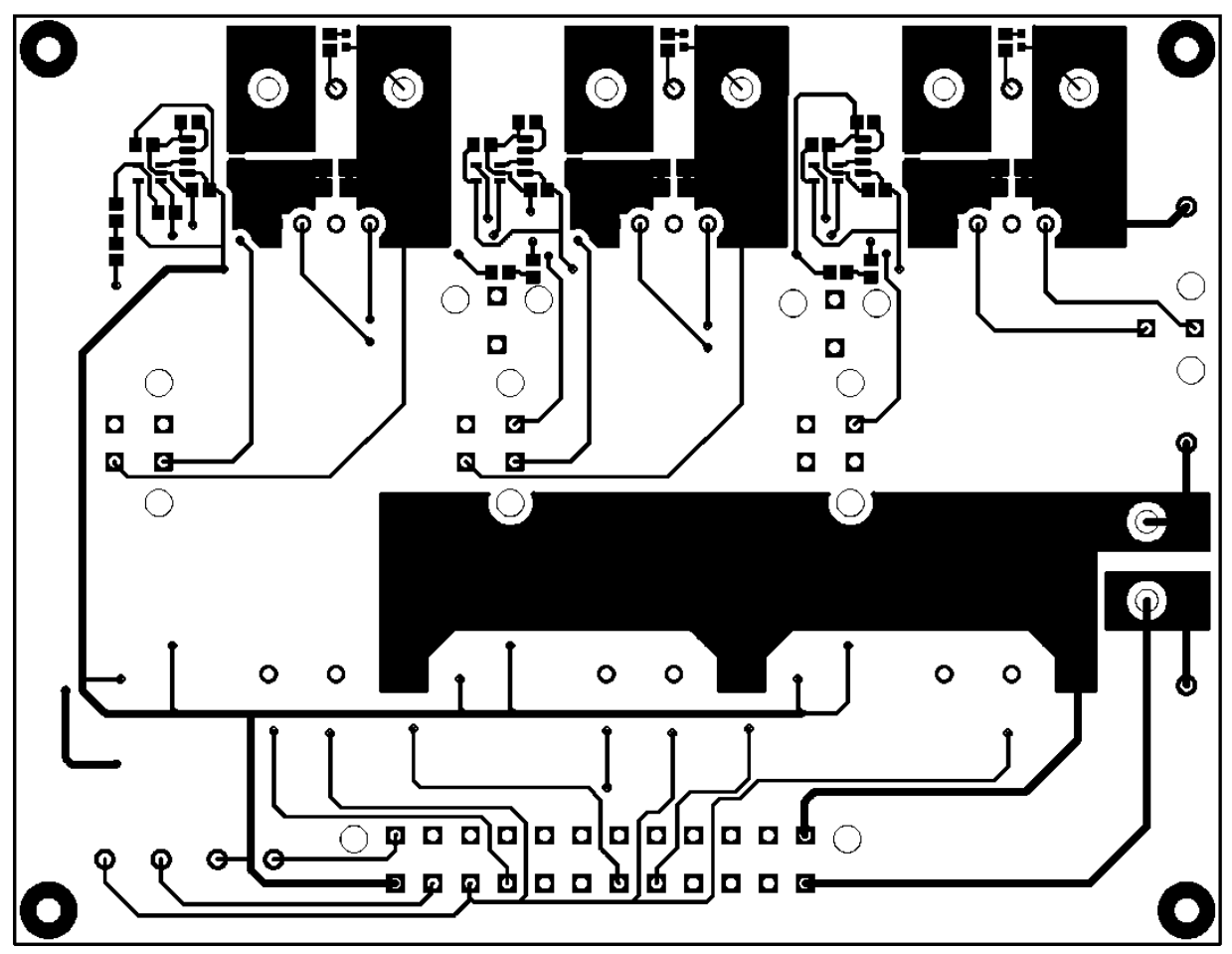

Figure 4-6. Power PCB Top Copper

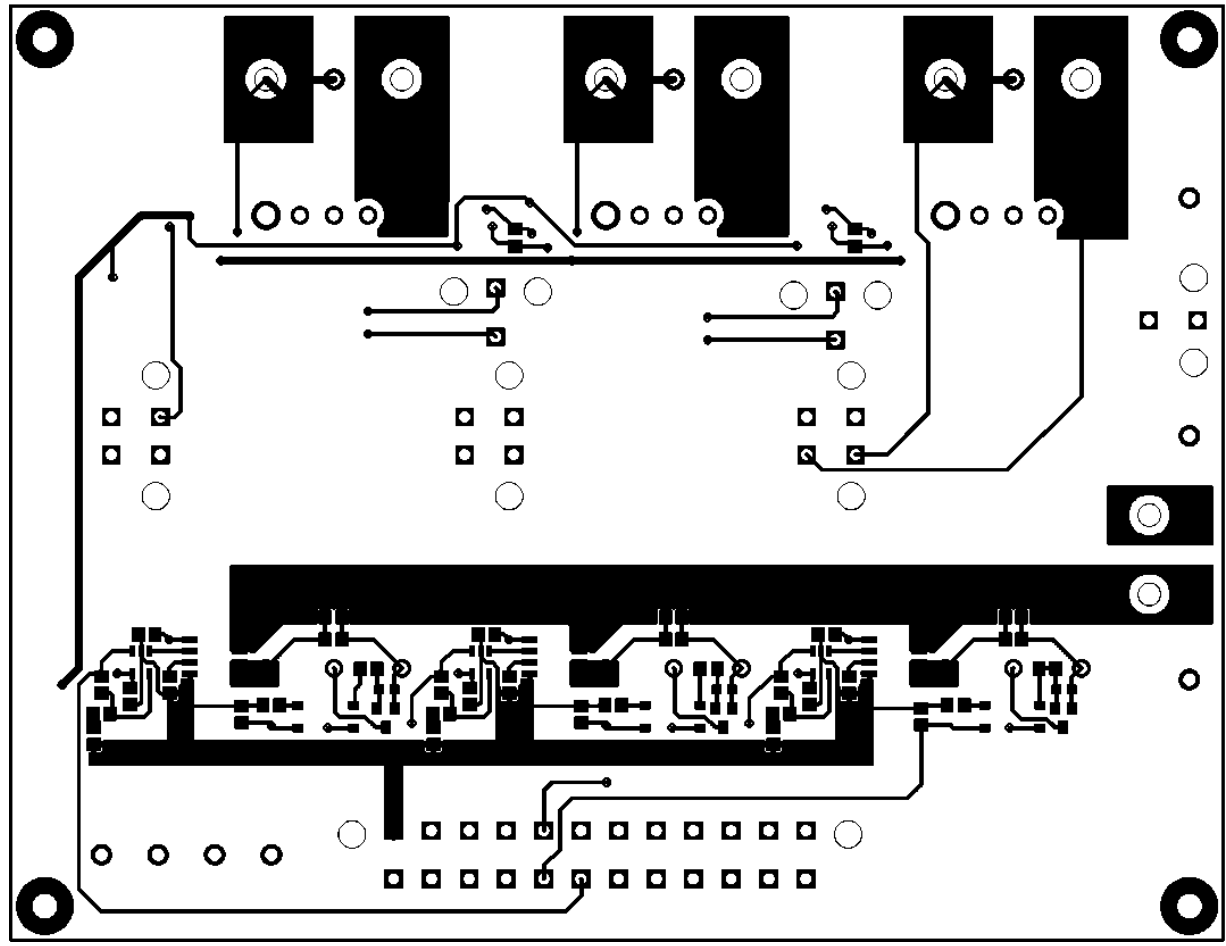

Figure 4-7. Power PCB Bottom Copper 


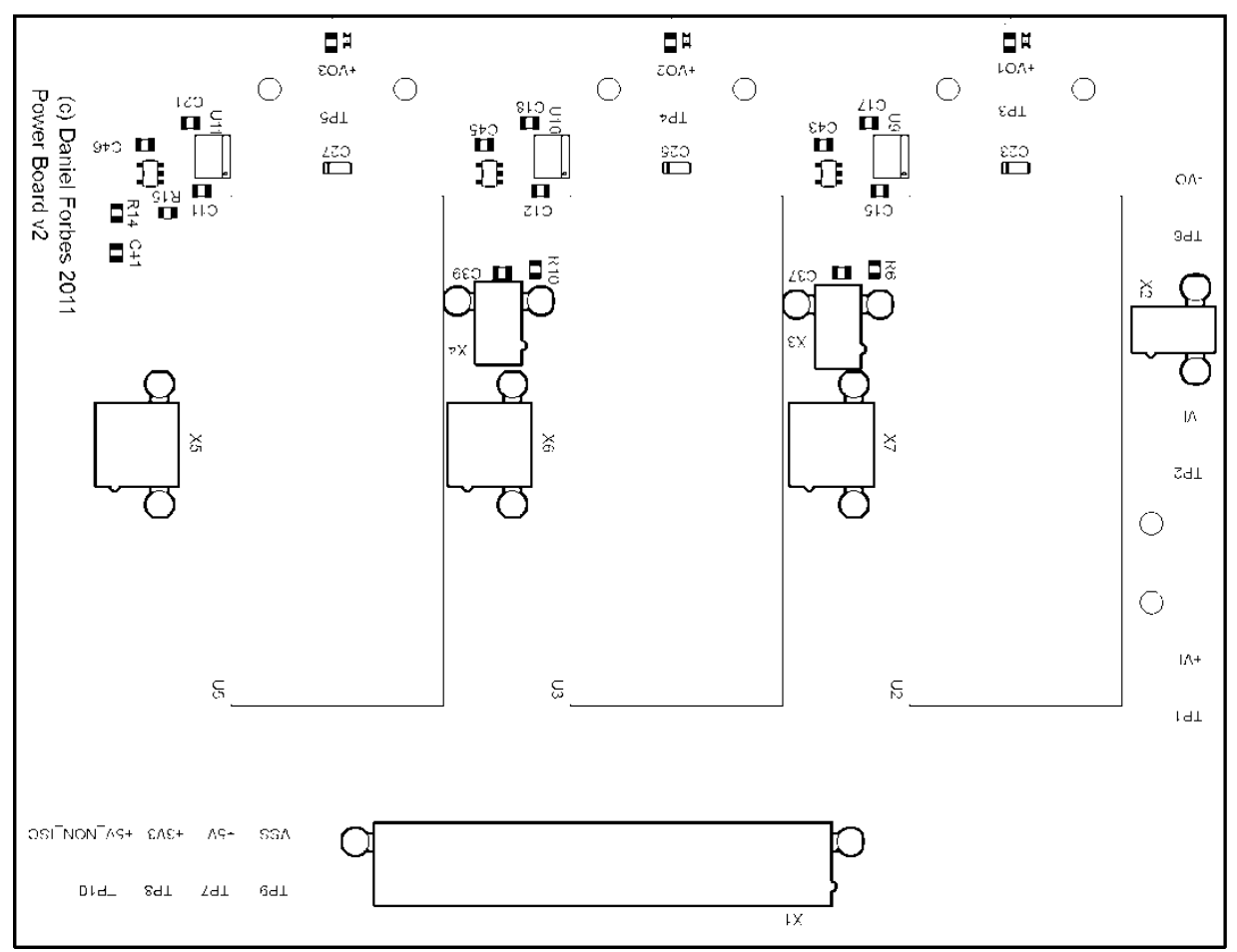

Figure 4-8. Power PCB Top Silkscreen

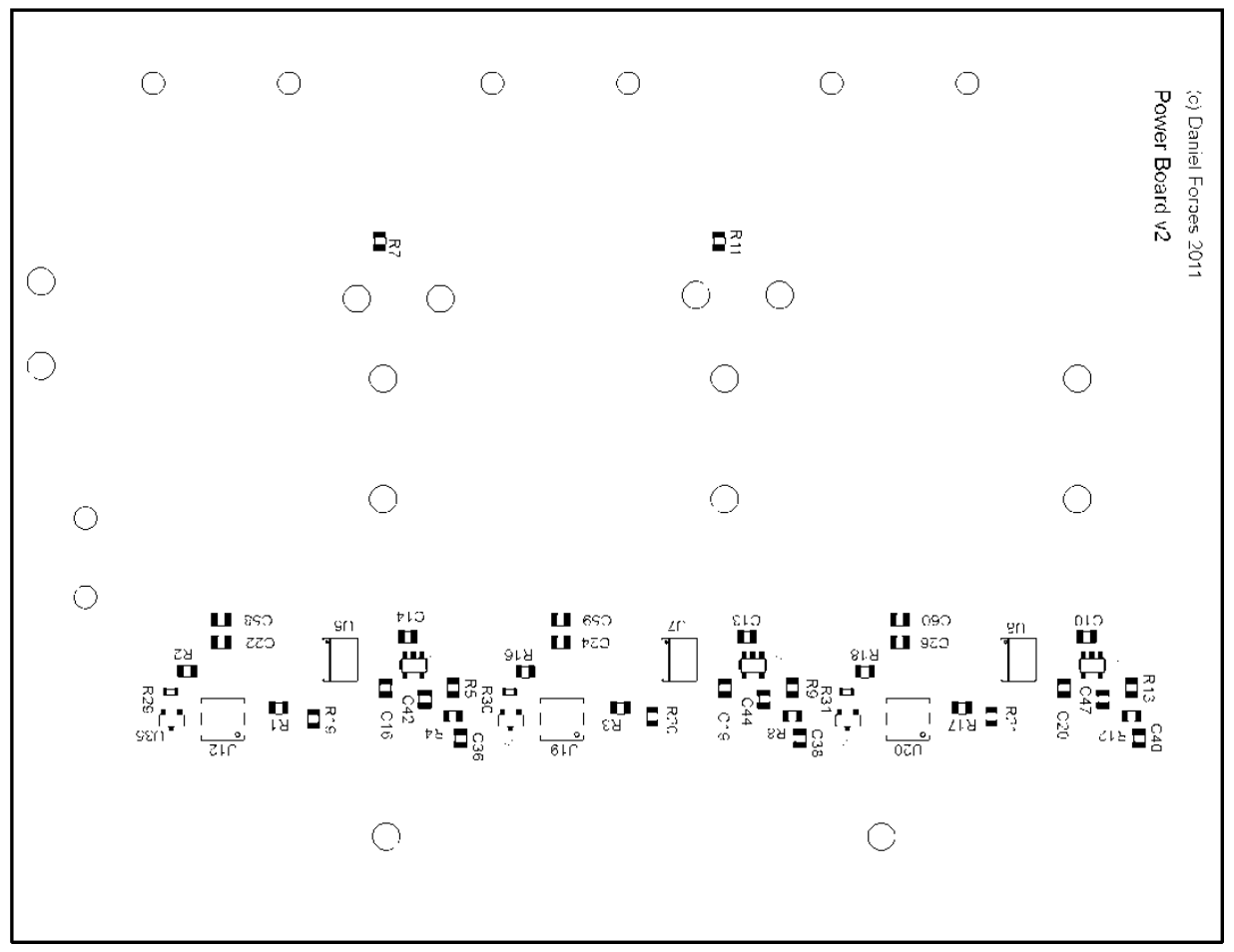

Figure 4-9. Power PCB Bottom Silkscreen (Mirrored) 


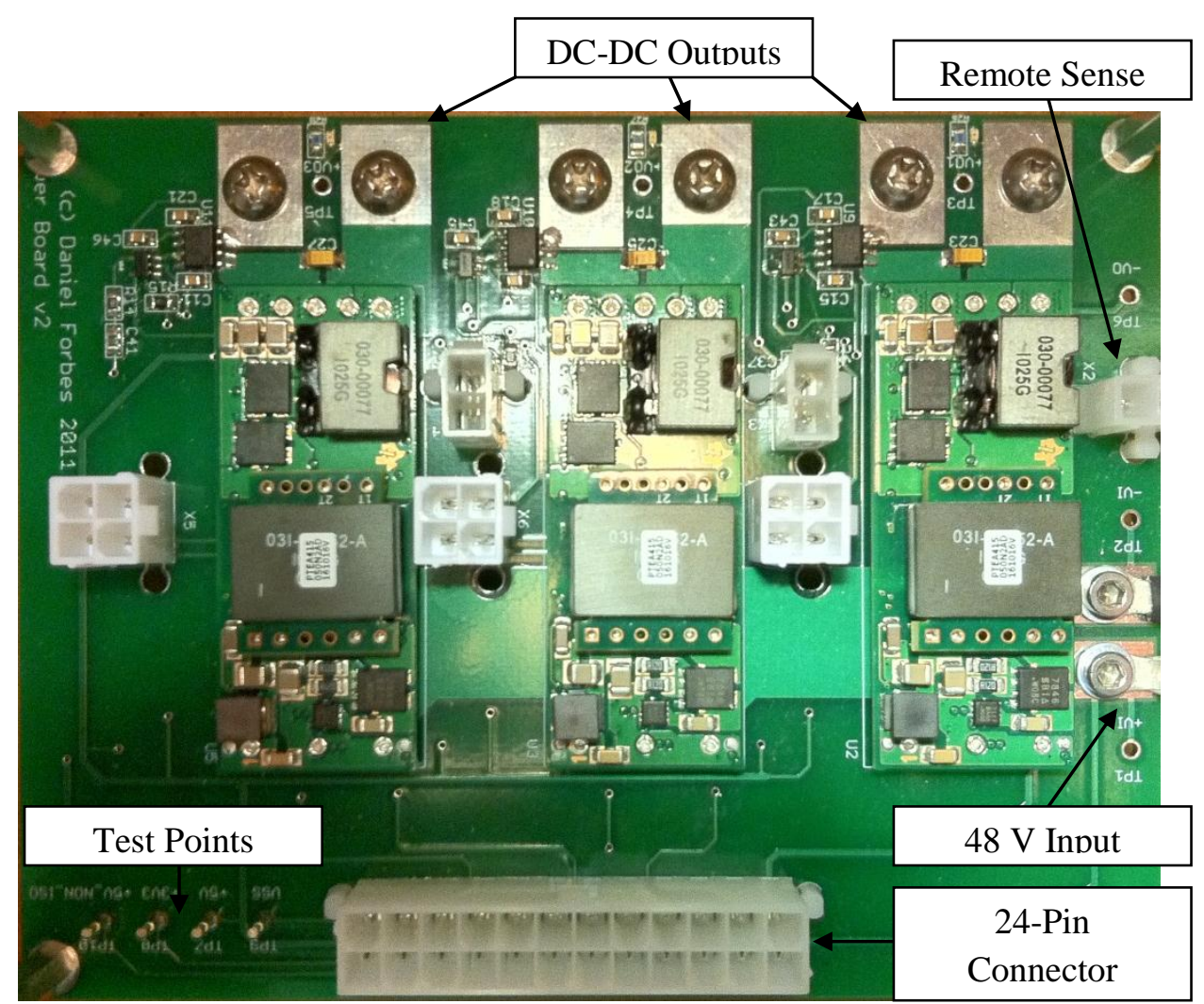

Figure 4-10. Power PCB Photo with Features Labeled

\subsection{Load-Share PCB}

Since the UCC39002EVM was purchased from TI, this thesis did not focus on its design. The UCC39002EVM User's Guide contains information regarding the load-share controller PCB design and layout [12]. Using the component values from the design section above, the UCC39002EVM PCB was populated and is shown in Figure 4-11 below. 


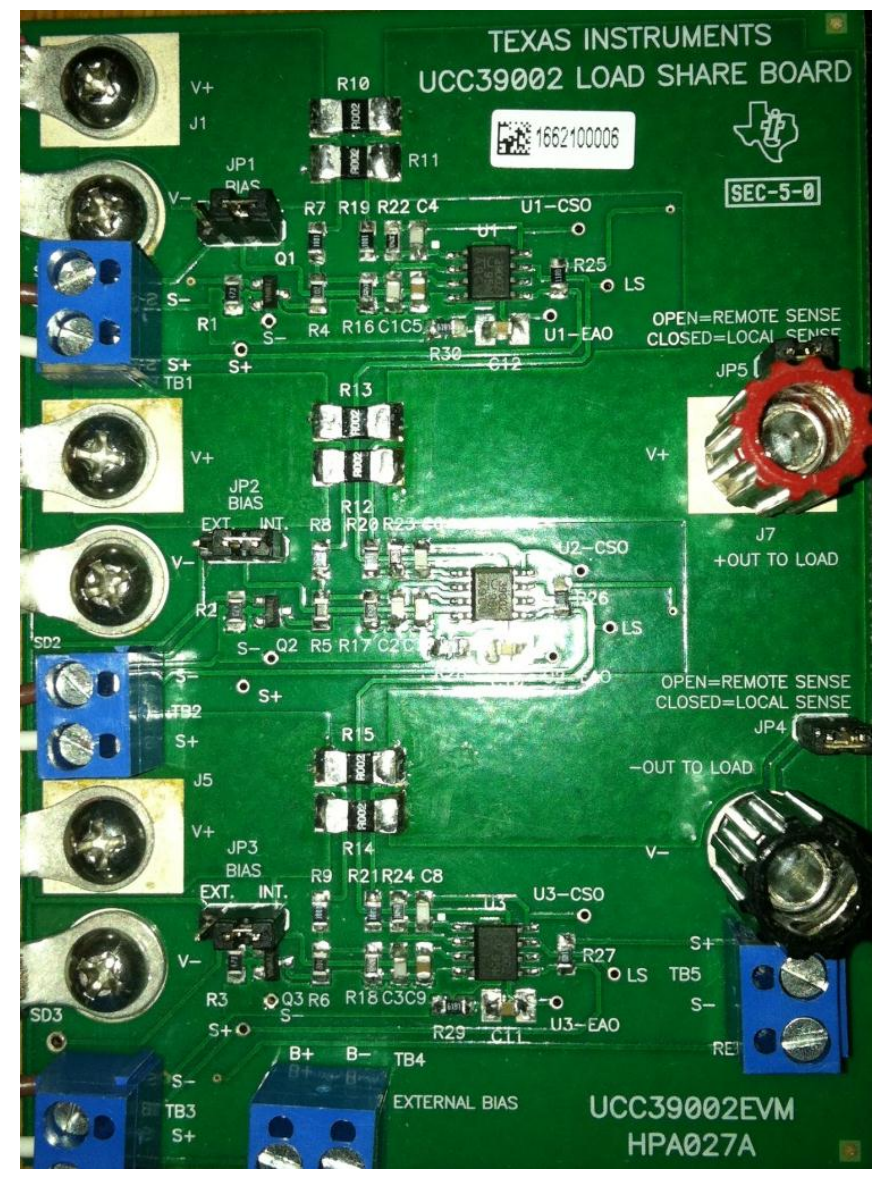

Figure 4-11. UCC39002EVM PCB 


\section{Chapter 5. Software Design}

In order for this thesis to function, various systems had to be programmed with custom software. Embedded firmware running on the microcontroller allowed for system data sampling and DC-DC converter control via a control algorithm. This firmware was programmed to send data to an accompanying piece of software running on a personal computer. This software displayed real-time data to an operator and also logged all data values. The sections below discuss in more detail both of these programs.

\subsection{Embedded Firmware}

The embedded firmware in this thesis runs on the PIC24FJ128GA006 microcontroller on the control PCB. It is written in the $C$ programming language and compiled in Microchip's MPLAB IDE using the $C 30$ compiler. The core firmware is contained within main.c, with supporting files adc.c, and uart1.c used to configure some of the microcontroller peripherals. The PIC24FJ128GA006 datasheet [14] was referenced for all peripheral configurations. The complete firmware code can be found in Appendix C.

\subsubsection{Analog-to-Digital Converter (ADC) Configuration}

Section 17 of the PIC24F Reference Manual [15] was used for reference when configuring the ADC. The file adc.c contains all the register configurations for the ADC. The ADC uses six status and control registers, and adc.c sets them to function properly in the hardware setup of this thesis. AD1CON1, AD1CON2 and AD1CON3 control the general operation of the ADC, AD1CHS selects which pins are passed into the ADC, AD1PCFG sets the ADC pins as analog (as oppose to digital), and AD1CSSL sets the pins to be included in an automatic scan. The $\mathrm{AD} 1 \mathrm{CON} 1$ register controls whether the $\mathrm{ADC}$ is enabled or not, and in order for the ADC to be configured it must be disabled. In this thesis, the ADC is set up to auto-convert all selected channels according to a timing sequence set by bits in AD1CON3. The PIC24FJ128GA006 has 
an analog module clock with a period defined as $\mathrm{T}_{\mathrm{AD}}$. As defined in the reference manual, an ADC conversion takes $12 \mathrm{~T}_{\mathrm{AD}}$. In order to calculate $\mathrm{T}_{\mathrm{AD}}$, the device instruction cycle clock period, $\mathrm{T}_{\mathrm{CY}}$ must be calculated. $\mathrm{T}_{\mathrm{CY}}$ is derived from the system clock defined earlier as $20 \mathrm{MHz}$, and is given in (5-1) below. Defining the ADC conversion clock period select bits in the $\mathrm{AD} 1 \mathrm{CON} 3$ register as $0 \mathrm{x} 0 \mathrm{~F}$ (or $16 \mathrm{~T}_{\mathrm{CY}}$ ) and using (5-2) gives $\mathrm{T}_{\mathrm{AD}}$.

$$
\begin{gathered}
T_{C Y}=\frac{2}{F_{O S C}}=\frac{2}{20 M H z}=0.1 \mu s \\
T_{A D}=T_{C Y}(A D C S+1)=0.1 \mu s(15+1)=1.6 \mu s
\end{gathered}
$$

Another important parameter in the ADC settings involves the SAMC bits in the AD1CON3 register. These bits control the length of time between the start of sampling and the start of conversion (essentially the sample time). Setting SAMC to 15 results in a sample time of $15 \mathrm{~T}_{\mathrm{AD}}$ or $24 \mu \mathrm{s}$.

\subsubsection{Serial Port (UART) Configuration}

The UART peripheral module in the PIC24FJ128006 is configured in uart1.c, which was adapted from code found in a PIC24 tutorial [16]. This configuration file also provides the serial communication functions used in main.c, including UART initiation, transmit and receive, along with some others. The serial port will be configured with the settings shown in Table 5-1.

Table 5-1. Serial Port Settings

\begin{tabular}{cc}
\hline Parameter & Value \\
\hline Baud Rate & 9600 \\
Data Bits & 8 \\
Stop Bits & 1 \\
Parity & None \\
Flow Control & None \\
\hline
\end{tabular}

\subsubsection{Main}

The overall control of the system in this thesis is implemented in main.c. This file deals with reading in and averaging different voltages and currents across the system, enabling and 
disabling DC-DC converters, and sending data over a serial line to a personal computer. Within main.c the majority of interesting code appears in the functions calibACS(), runA2D(), and railControl(). The remainder of the code deals with setting up the microcontroller ports, initializing peripherals, and other background tasks. After settings are made and variables are declared, main.c enters an endless while() loop, where it stays until power is removed or a reset is detected. Just prior to entering the while() loop, main.c calls calibACS(). calibACS() is set up to determine the exact value of the offset voltages of the six ACS713 current sensors in the thesis. It does this by disabling all three DC-DC converters (to assure 0 A through the DC-DC), and then calling run $\mathrm{A} 2 \mathrm{D}()$ and logging the measured offset voltages.

When the function run $\mathrm{A} 2 \mathrm{D}()$ is run, it reads all enabled $\mathrm{ADC}$ channels and records the results. Depending on the value of ADC_avg, a running average of these values is calculated. The current setting of ADC_avg is 16, meaning each value output from runA2D() to the rest of the program is an average of 16 consecutive readings. Once an averaged value of each enabled channel is acquired, runA2D() outputs all the data to the serial port (which the PC software will read in, discussed in the next section). With this information available, railControl() can now be called in the while() loop.

The function railControl() looks at the real-time current (averaged) of each DC-DC, and determines how many DC-DC converters should be enabled. At the time of this report, the control scheme is simple; if the current through the first DC-DC is over $9 \mathrm{~A}$, turn on the next converter. Similarly, if the current though the first or second DC-DC is over 9 A, turn on the third converter. Similarly, if all three DC-DCs are on and the total current is $18 \mathrm{~A}$, turn off the third converter, etc. In the future work section below, a more sophisticated control scheme is proposed. 


\subsection{PC Software}

The PC software developed for this thesis provides a graphical user interface (GUI) and a means to record data from the system. The software was developed using the $\mathrm{C} \#$ programming language in Microsoft Visual C\# 2010 Express. The complete software code can be found in Appendix D. Figure 5-1 shows the GUI before it is populated with any data. The GUI allows the user to select a COM port that the hardware is connected to, open or close the serial connection, and override the ACS713 current sensor op-amp gain values used to calculate current. The GUI also displays input and output voltage and current for the system and for each DC-DC, as well as efficiency. ACS713 offset voltages and gains are displayed, and a check-box indicates whether or not a certain DC-DC is enabled. Bar graphs also display the output current of each converter as well as the total system output current, to give a visual of where the system is operating in relation to minimum and maximum load. In the background, the software records all data into a *.csv file for later use. All data is displayed and recorded once a second. 


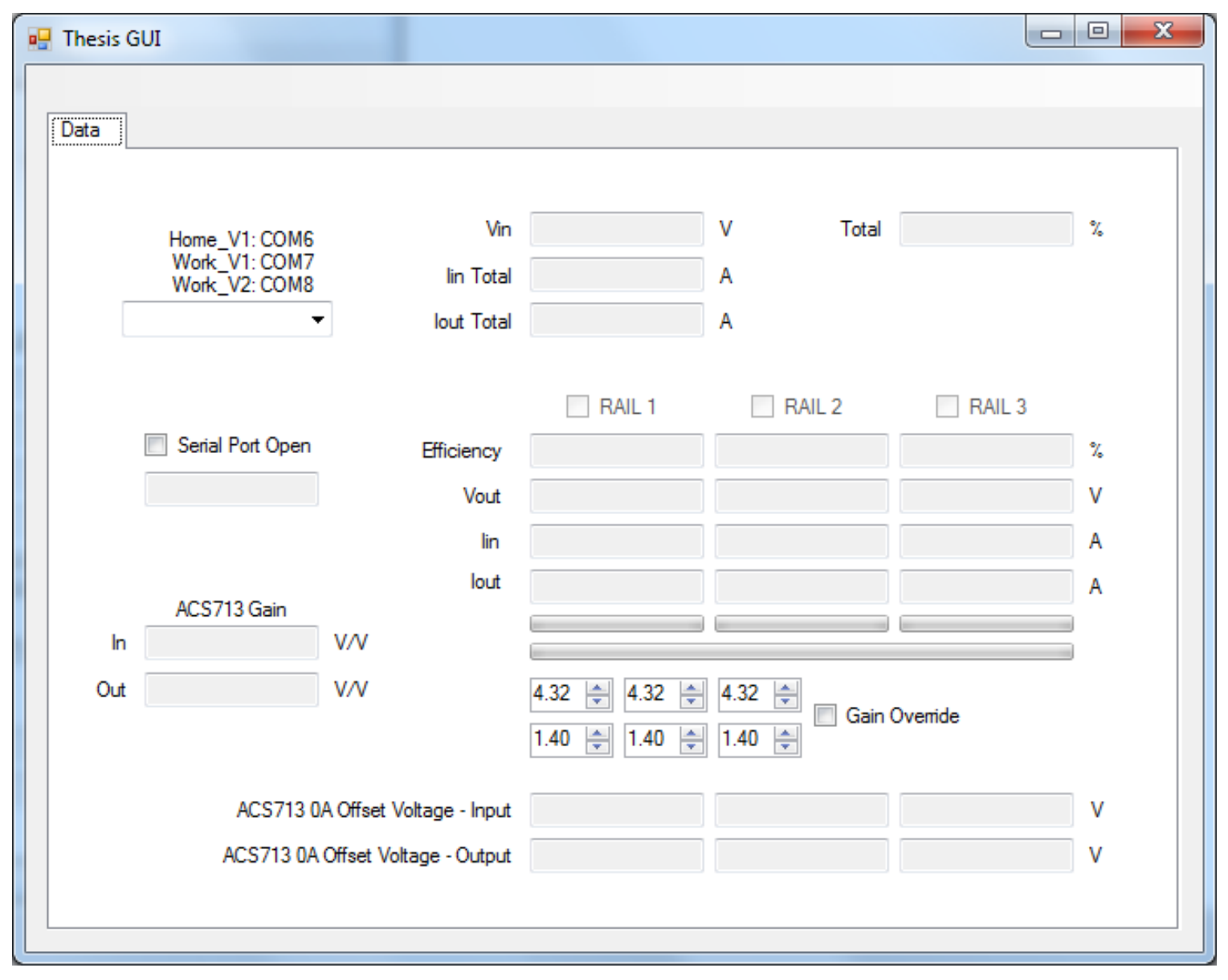

Figure 5-1. PC Software GUI

As mentioned, the GUI displays the ACS713 gain (gain of the op-amp at the output of the ACS713) and provides a means to override the gain with user-selectable values. This enables tuning of the system, where-in the proper value can be found and entered back into the microcontroller firmware for a more accurate system. Any data that is displayed in the GUI can later be retrieved from a file, located in the root directory of the system hard drive, called TestFile.csv. This file is only available once the serial port has been closed or the GUI has been terminated. The file contains appropriate field headers and time-stamps (taken from the computer system clock) of when the data was taken. 


\section{Chapter 6. Hardware Results and Analysis}

In order to perform tests on the hardware in this thesis, first bring-up and calibration of the system was required, then load-testing and tuning. For all of this work, some test instruments were required. A 48 VDC power supply was needed to power the system, an electronic load was required to draw power from the system, a personal computer was needed to run the software and compile data, and an oscilloscope and digital multimeter (DMM) were used for general diagnosis.

Table 6-1 shows the details of the instruments used.

Table 6-1. Test Equipment Used for System Analysis

\begin{tabular}{cccc}
\hline Instrument & Manufacturer & Part Number & Information \\
\hline DC Power Supply & Densei-Lambda & JWS240P-48 & 48V, 5A (10A Peak) \\
Electronic Load & Kikusui & PLZ-152W & 150W Load Max. \\
Personal Computer & HP and Dell & N/A & Windows XP \\
Oscilloscope & Tektronix & TPS2024 & 4-Channel, Isolated \\
Digital Multimeter & Fluke & 287 & Full-function DMM \\
\hline
\end{tabular}

\subsection{Calibration}

Since the hardware in this thesis has many sensors, both current and voltage, there are design constants in the firmware which, when implemented in hardware, are slightly different and must be measured. As discussed in the firmware section above, the 0A offset voltage of the ACS713 is measured by the firmware each time the system is powered up or reset. Other values, such as the op-amp gain of the output stage following the ACS713 had to be measured for verification. This was done running the system with different loads and measuring the gains with the DMM. Once these values were measured, the firmware was updated to reflect the actual gains, rather than the ideal. Another calibration needed was on the linear optocoupler used to isolate the input and output voltage measurements from the microcontroller. In order for this isolation to work, a linear relationship had to exist between the input and output of the optocoupler. To show this relationship, the input to the optocoupler was varied from $0 \mathrm{~V}$ to $3 \mathrm{~V}$ and the output voltage was recorded. Table 6-2 and Figure 6-1 show the data taken during calibration. As can be seen from 
Figure 6-1, the LOC110 linear optocoupler has a very linear relationship between its input and output voltage.

Table 6-2. LOC110 Linear Optocoupler Linearity Check

\begin{tabular}{ccc}
\hline $\mathrm{V}_{\text {IN }}(\mathrm{V})$ & $\mathrm{V}_{\text {OUT (Calculated) }}(\mathrm{V})$ & $\mathrm{V}_{\text {OUT (Measured) }}(\mathrm{V})$ \\
\hline 0 & 0 & 0.02 \\
0.2 & 0.2 & 0.22 \\
0.4 & 0.4 & 0.41 \\
0.6 & 0.6 & 0.61 \\
0.8 & 0.8 & 0.80 \\
1 & 1 & 0.99 \\
1.2 & 1.2 & 1.17 \\
1.4 & 1.4 & 1.36 \\
1.6 & 1.6 & 1.55 \\
1.8 & 1.8 & 1.73 \\
2 & 2 & 1.92 \\
2.2 & 2.2 & 2.11 \\
2.4 & 2.4 & 2.30 \\
2.6 & 2.6 & 2.50 \\
2.8 & 2.8 & 2.68 \\
3 & 3 & 2.88 \\
\hline
\end{tabular}

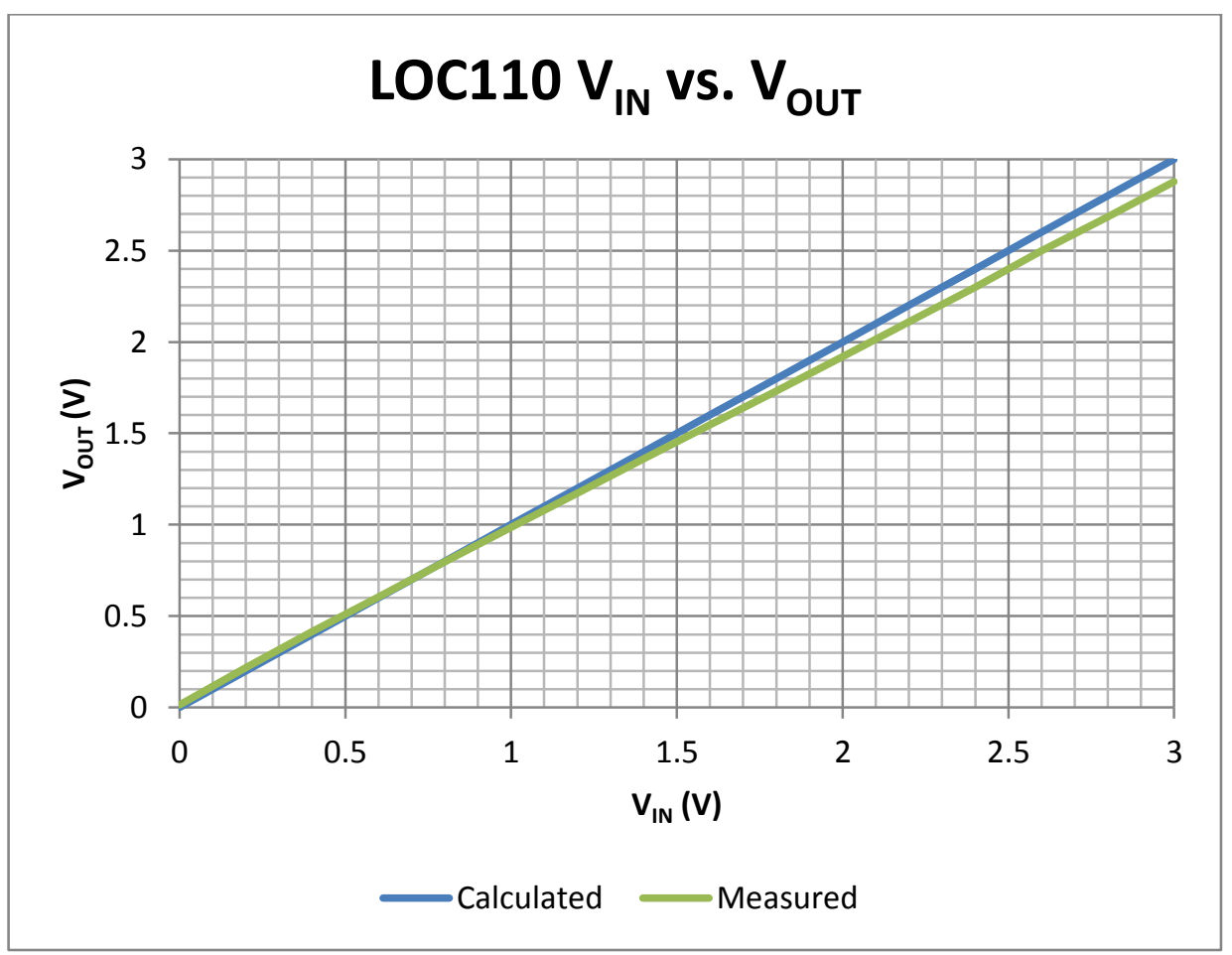

Figure 6-1. LOC110 Linear Optocoupler Linearity Calibration 


\subsection{Load Testing and Tuning}

After calibration and firmware tweaking was performed, the system was ready for various tests, including full-load testing. Enabling the control system on the microcontroller and running the electronic load on the output of the load-share controller, the output current was increased from 0 A to 26 A (1 A below full system capability). During this test, the software was running on a computer and logging hardware data. After this test, the data was analyzed and produced the following graphs. Figure 6-2 shows the load currents of each DC-DC converter as compared to the total system output current. As shown, from 0 A to 9 A only one converter was enabled and the current ramps up as expected. At around the 9 A mark the second converter was enabled and the current was evenly shared by the two converters. Similarly, just before the total load current reached $18 \mathrm{~A}$ the third converter was enabled and the current was shared by all three converters. This was all done under the control of the microcontroller and shows that the system was acting as expected; the microcontroller was reading in values correctly and sending proper control signals, and the load-share controller was commanding the converters to share the load evenly.

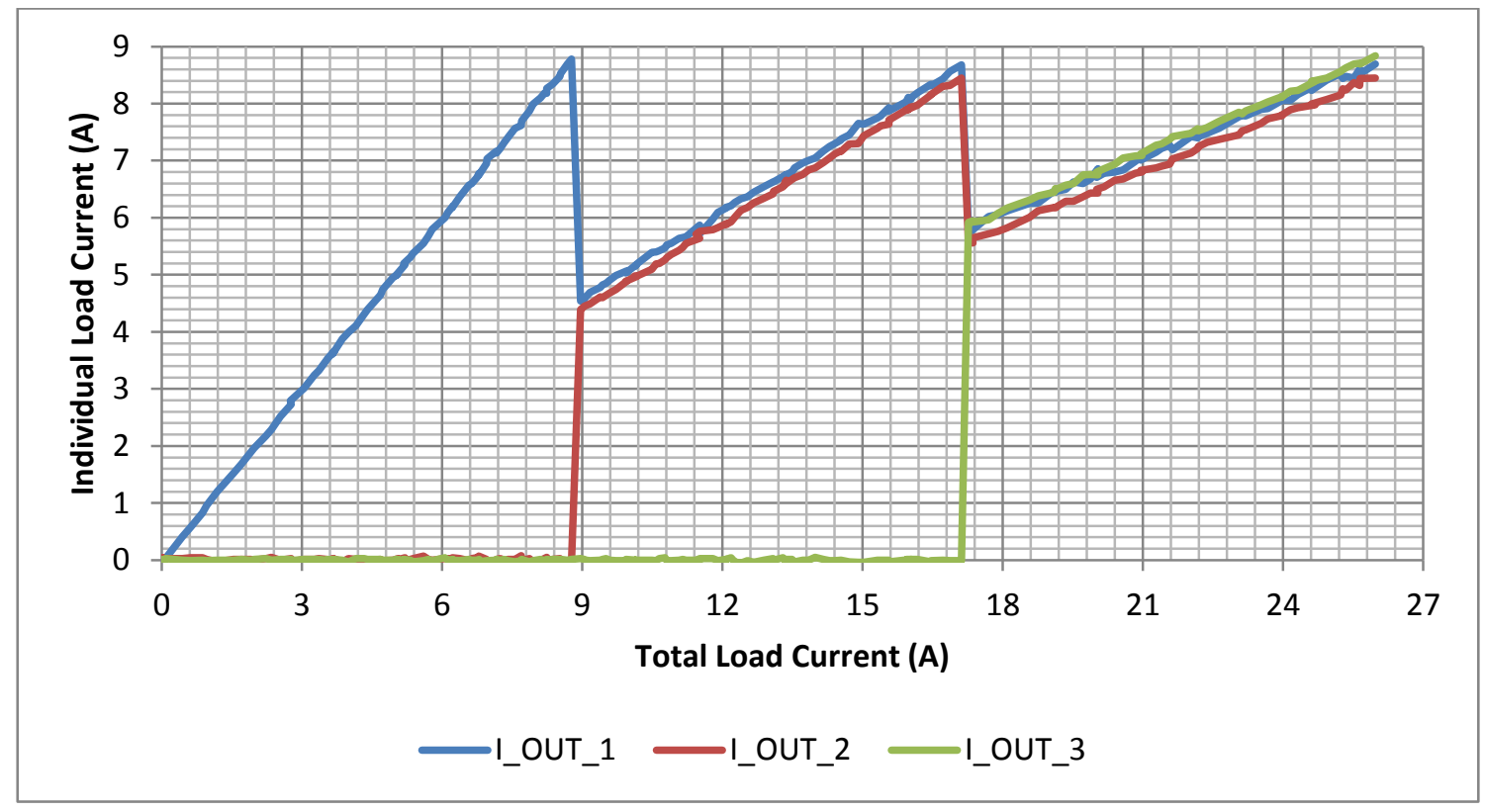

Figure 6-2. Load Currents of Each DC-DC Converter During Full-Load Test 
From this same data, an efficiency curve was generated as shown in Figure 6-3. As in the simulation results, the switch points in Figure 6-3 are evident by the step decreases in efficiency near $9 \mathrm{~A}$ and $18 \mathrm{~A}$ (because of the graph scale they look less pronounced). Adjusting the switch points to $12 \mathrm{~A}$ and $24 \mathrm{~A}$ (as in the simulation improved parallel converter) gives a smoother efficiency curve without the steps in efficiency. This is also shown in Figure 6-3 below.

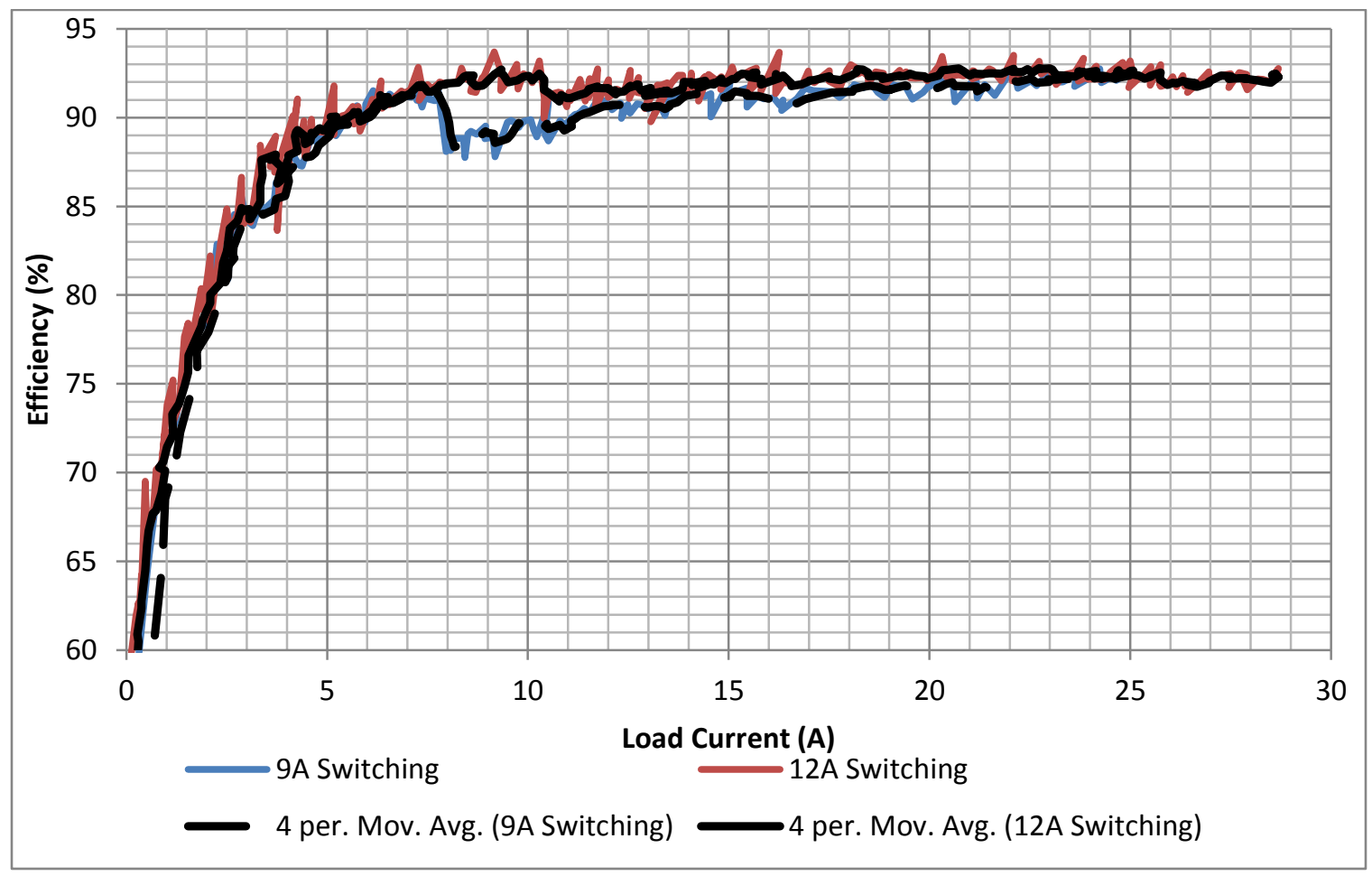

Figure 6-3. Efficiency Curve for Generated from Full-Load Test

Figure 6-4 and Figure 6-5 show the single DC-DC converter used a control case and the complete digitally-controlled parallel DC-DC converter designed and built for this thesis. Figure 6-6 and Figure 6-7 show the test bench setup used druing testing and data collection. 


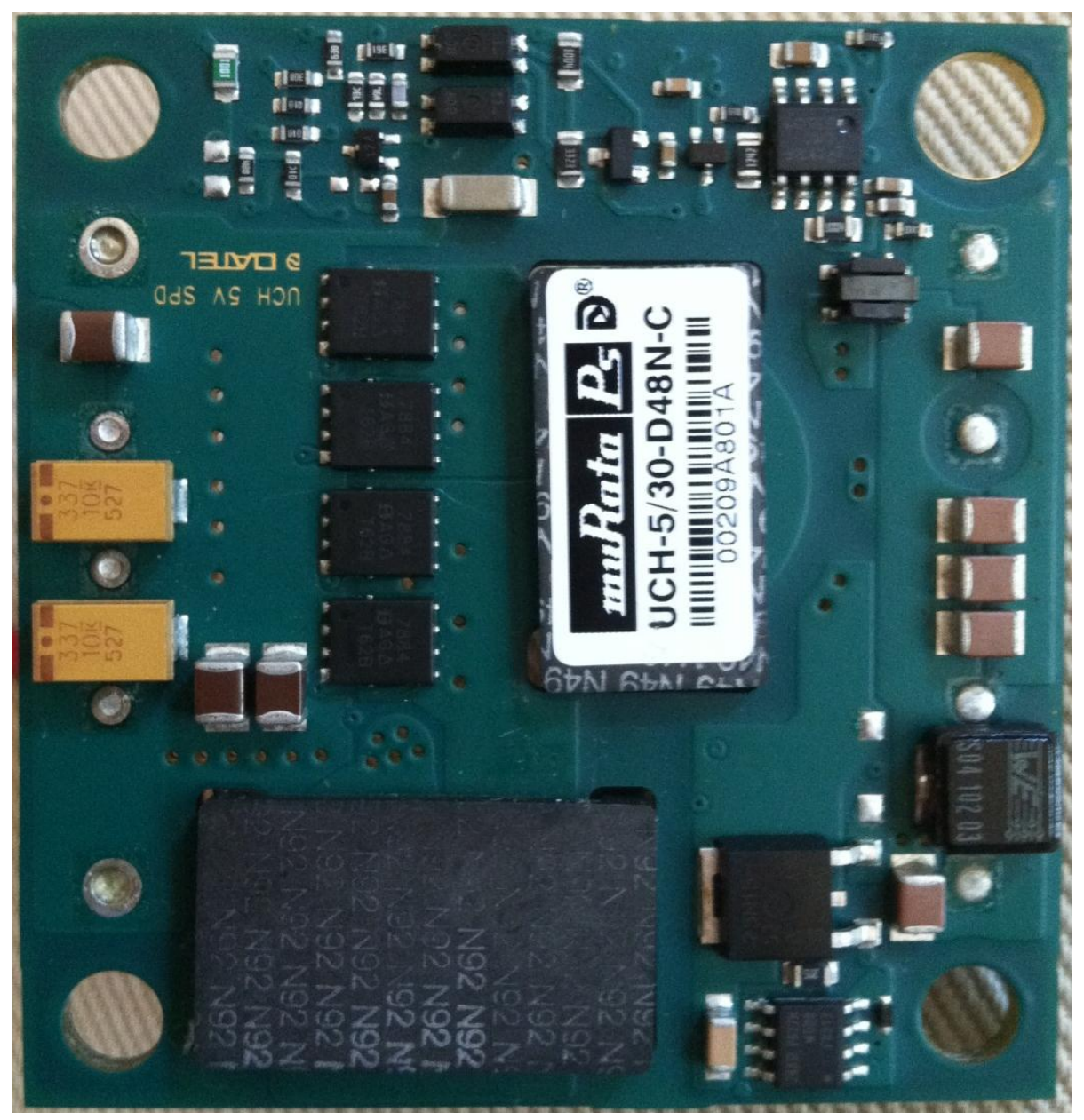

Figure 6-4. Single DC-DC Converter Used as Control Case 


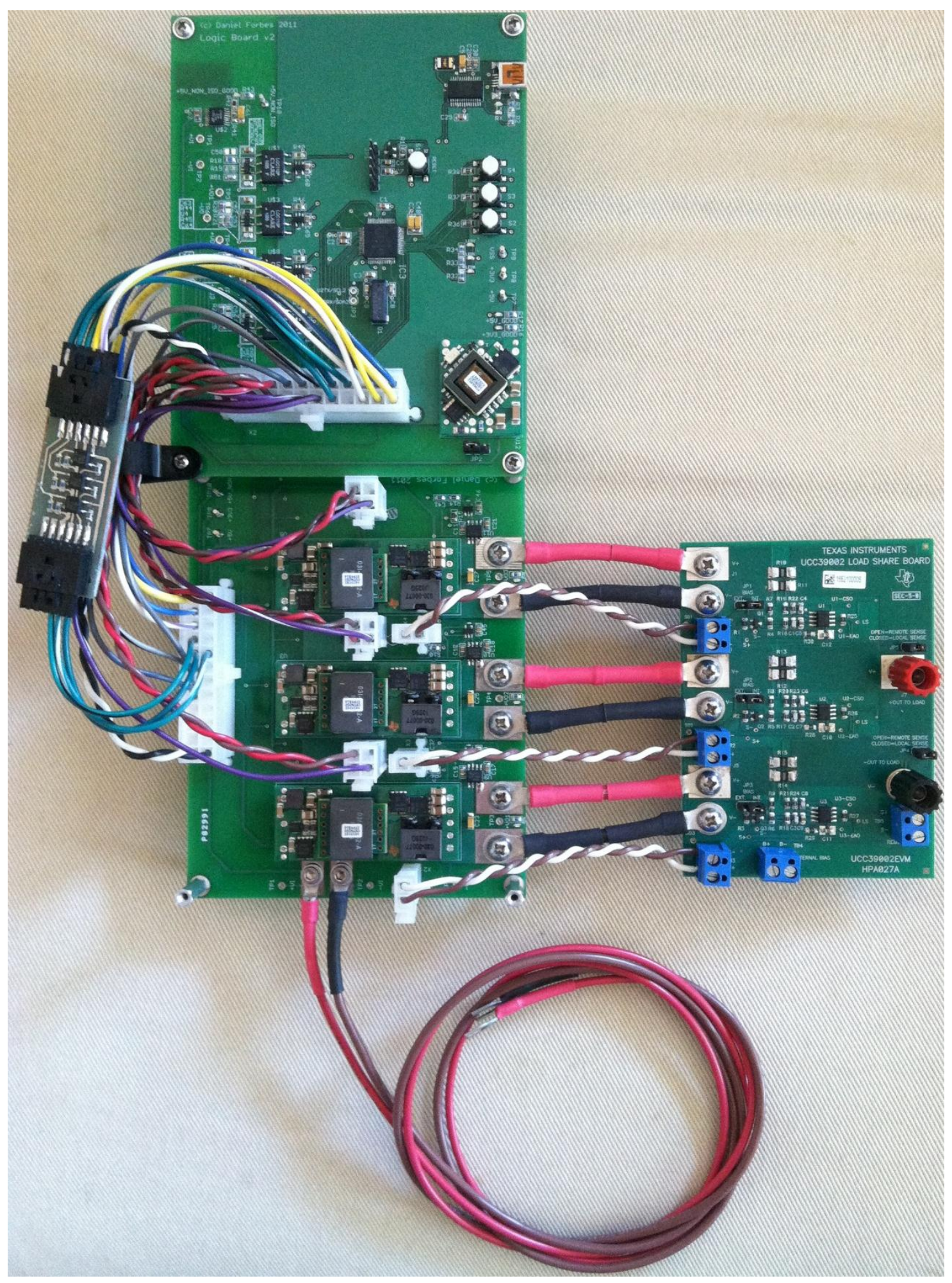

Figure 6-5. Complete Digitally-Controlled Parallel DC-DC Converter System 


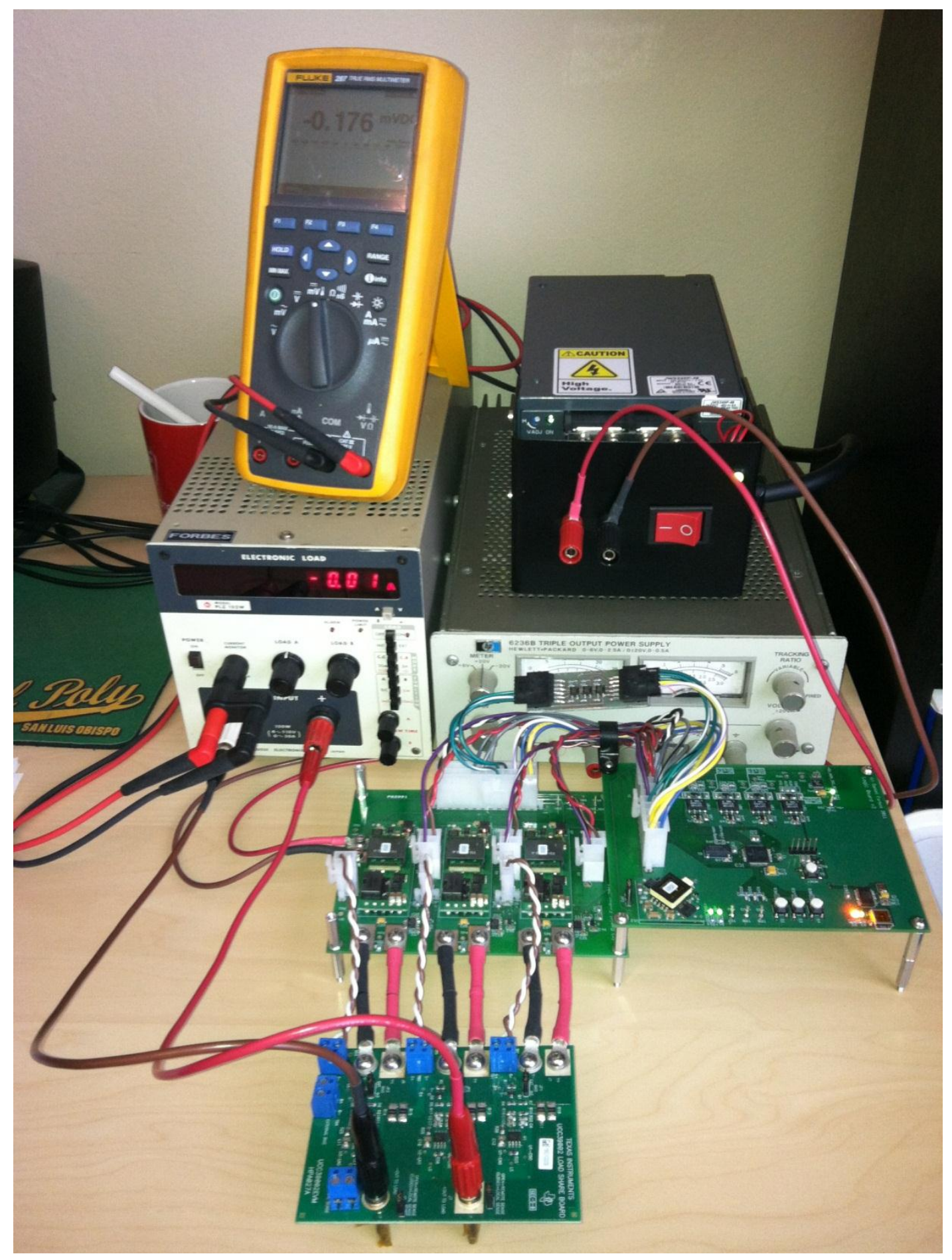

Figure 6-6. Test Bench Setup with Thesis System Attached 


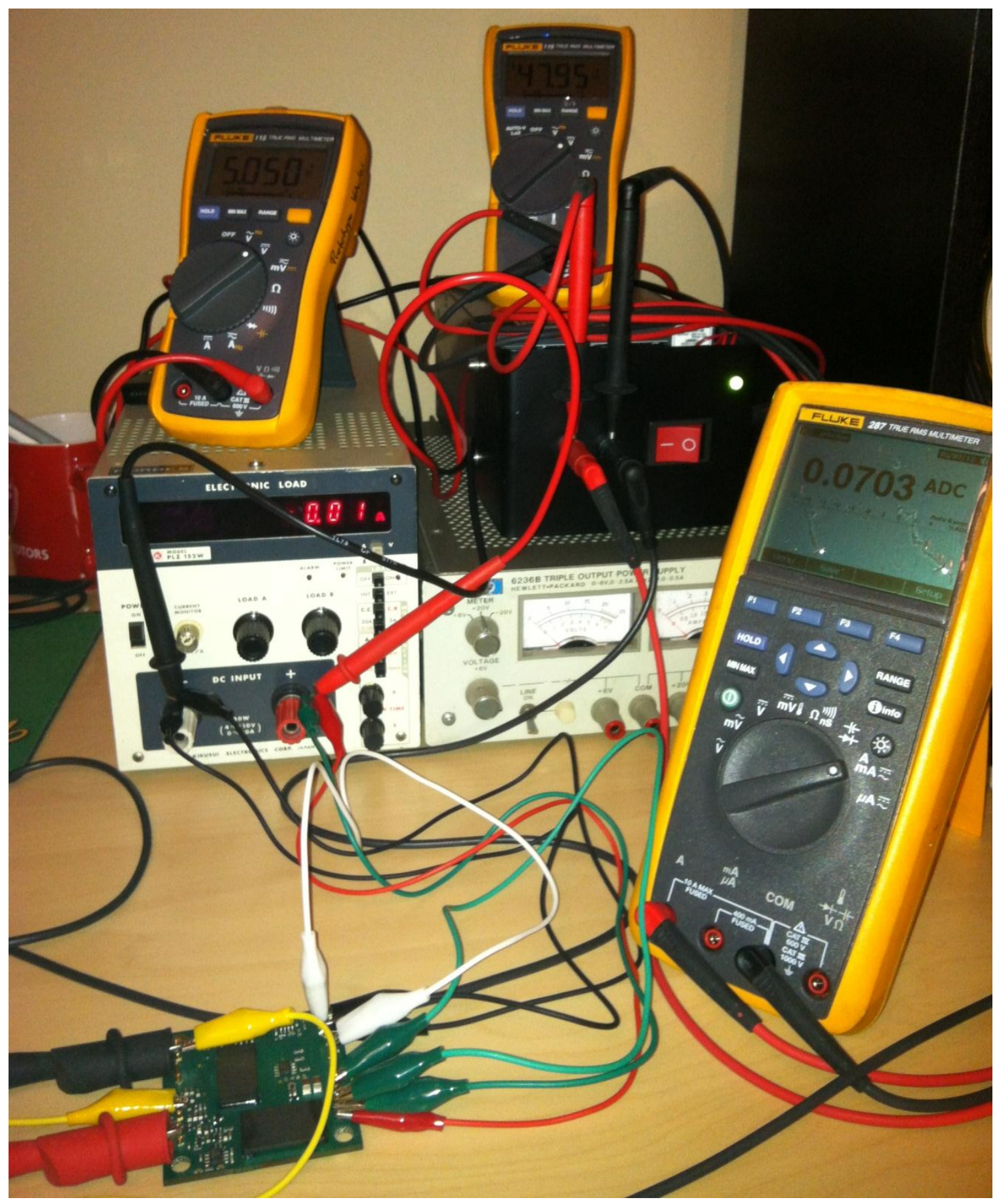

Figure 6-7. Testing Single DC-DC Converter (Control Case) 


\section{Chapter 7. Conclusion}

Given the need for especially improved low-load efficiency in DC-DC converters, a digitallycontrolled parallel DC-DC converter system was proposed. A MATLAB Simulink model was developed to simulate a system with an arbitrary converter topology. This model simply required the efficiency curve of a DC-DC converter and some desired operating characteristics to run. The results of the simulation showed that indeed the proposed parallel system out-performed a control-case single converter in the low-load case while maintaining full-load efficiency. The Simulink model provided a tool to develop a control algorithm which could later be implemented in a hardware design.

Following the successful simulation, a hardware implementation was proposed, based around three off-the-shelf DC-DC converters. The design implemented digital control via a microcontroller and used various current and voltage sensors to feed information to the control scheme. A load-share controller was designed into the output of the paralleled DC-DC converters to ensure equal load on all enabled converters. Along with the hardware system a software interface was developed to provide real-time information to a user as well as to record system data.

The efficiency results of the hardware system showed an improvement over the single converter control case, and performed close to what simulations predicted. Figure 7-1 shows the efficiency results of the simulation (improved parallel and control) and the hardware (with an averaged trendline for the improved parallel system). The slight efficiency gain of the improved parallel hardware results over the simulated improved parallel system appear to be from a small offset error introduced in the hardware, and could be corrected with further development. From Figure 7-1 a comparison of the parallel hardware system can be made to the single control DCDC converter hardware. At lower loads (between $4.5 \mathrm{~A}$ and $8 \mathrm{~A}$ ), the parallel systems shows 
approximately a $2.2 \%$ increase in efficiency over the control case. At the higher end, again the parallel system shows an improvement of approximately $1.6 \%$. In the mid-range load, the systems are quite comparable with the parallel system showing a slight improvement overall. As just mentioned, the parallel system measurements appear to be slightly high compared to what was expected, but even with corrections this system should still outperform the control case.

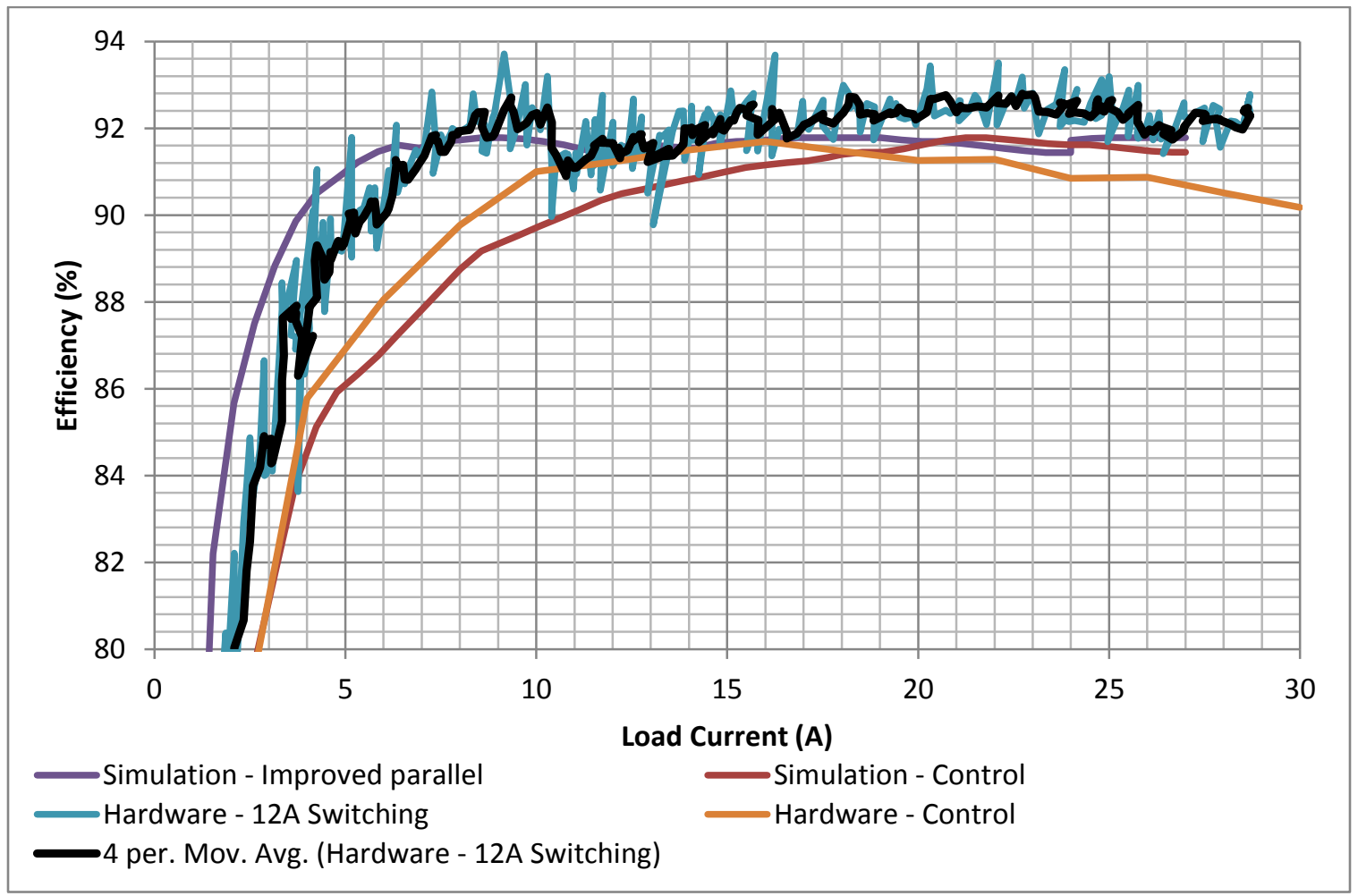

Figure 7-1. Efficiency Curves from Simulation and Hardware

Through the design of the hardware many issues arose in relation to an isolated system. Initial ground noise proved devastating for the design, and the redesigned system showed much improvement. PCB layout considerations in regards to trace width, trace spacing, and noise shielding played an important role in the correct workings of this thesis. 


\subsection{Future Work}

There are a few areas where further development could improve this thesis and make it more useful for the real world. To start with, sensed voltage and current values appeared to vary over time as the system was used, and this led to some erroneous data. To mitigate this in the current design, manual calibrations were performed, but this proved tedious and not always accurate. In a future design a more robust calibration would allow for more accuracy and tighter control of the system. Another issue that arose in hardware testing was a problem with converters shutting down when not specifically commanded to do so. This would arise when a different converter was shut down, and appeared to be caused by some noise levels on the output voltage line feeding back into the enable lines of the converter not commanded to shut down. Finding solutions to these two issues would greatly improve the reliability of the system as a whole.

Another area for future work involves adding stress considerations into the control algorithm. This could include DC-DC converter temperature (temperature sensors would need to be added in hardware), converter on-time, and also lifetime converter on-time. This level of control would allow for all the converters to be equally utilized and for improved robustness and durability. Additionally, a spare converter could be designed into the system and could be brought automatically online in the case of a failure of an operating converter. One possible control scheme bringing these metrics together could be Fuzzy Logic. This scheme would allow for a completely defined system over variable temperature, load, and on-time conditions. This scheme could also improve the efficiency curve by choosing the proper number of converters to enable to achieve maximum efficiency (in a more adaptable way than the currently employed control scheme). With further developments, this system can show improvements in reliability and efficiency over a conventional DC-DC converter. 


\section{Bibliography}

[1] H. H. C. Iu and C. K. Tse, "Study of Low-Frequency Bifurcation Phenomena of a ParallelConnected Boost Converter System Via Simple Averaged Models," IEEE Transactions on Circuits and Systems, vol. 50, no. 5, pp. 679-686, May 2003.

[2] K. Rinne, A. Kelly and E. O'Malley, "A Novel Digital Single-Wire Quasi-Democratic Stress Share Scheme For Paralleled Switching Converters," Limerick, Ireland, 2010.

[3] S. Luo, Z. Ye, R.-L. Lin and F. C. Lee, "A Classification and Evaluation of Paralleling Methods for Power Supply Modules," Power Electronics Specialists Conference, 1999. PESC 99. 30th Annual IEEE, vol. 2, pp. 901-908, 1999.

[4] L. Tang and S. Gui-Jia, "A Low-Cost, Digitally-Controlled Charger for Plug-In Hybrid Electric Vehicles," Energy Conversion Congress and Exposition, 2009. ECCE 2009. IEEE, pp. 3923-3929, Sept. 2009.

[5] S. M. Wang, M. W. Cheng, Y. S. Lee, R. H. Chen and W. T. Sie, "Intelligent Charged System for Lithium-Ion Battery Strings," in Telecommunications Energy Conference, 2009. INTELECT 2009. 31st International, Incheon, 2009.

[6] A. E. Elsis and M. T. Aydemir, "Hot-Swap Parallel Operation of Boost Converters Designed for the Output of Fuel Cells," in Electrical Machines and Power Electronics, 2007. ACEMP '07. International Aegean Conference on, Bodrum, 2007.

[7] H. Iu and C. K. Tse, "Bifurcation Behavior in Parallel-Connected Buck Converters," IEEE Transactions on Circuits and Systems-I: Fundamental Theory and Applications, vol. 48, no. 2, pp. 233-240, Feb. 2001.

[8] Clare, An Ixys Company, "LOC110 Single Linear Optocoupler," 19 November 2010. [Online]. Available: http://www.clare.com/home/pdfs.nsf/www/LOC110.pdf/\$file/LOC110.pdf.

[9] Clare, An Ixys Company, "Application Note AN-107, Linear Optocouplers," 25 June 2002. [Online]. Available: http://www.clare.com/home/pdfs.nsf/www/AN-107.pdf/\$file/AN107.pdf.

[10] Texas Instruments, "15-A, 48-V INPUT, 5-V OUTPUT, ISOLATED, 1/8th BRICK DC/DC CONVERTER," November 2010. [Online]. Available: http://www.ti.com/lit/ds/symlink/ptea415050.pdf. 
[11] Allegro MicroSystems, Inc., "Fully Integrated, Hall Effect-Based Linear Current Sensor IC with 2.1 kVRMS Isolation and a Low-Resistance Current Conductor," 12 October 2011. [Online]. Available: http://www.allegromicro.com/Products/Current-Sensor-ICs/Zero-ToFifty-Amp-Integrated-Conductor-Sensor-ICs/ /media/Files/Datasheets/ACS713Datasheet.ashx.

[12] Texas Instruments, "UCC39002 Advanced Load-Share Controller User's Guide, HPA027A," 9 February 2004. [Online]. Available: http://www.ti.com/lit/ug/sluu166a/sluu166a.pdf.

[13] B. "PCB Trace Width Calculator," 31 January 2006. [Online]. Available: http://circuitcalculator.com/wordpress/2006/01/31/pcb-trace-width-calculator/.

[14] Microchip Technology, Inc., "PIC24FJ128GA010 FAMILY," January 2012. [Online]. Available: http://ww1.microchip.com/downloads/en/DeviceDoc/39747F.pdf.

[15] Microchip Technology, Inc., "PIC24F Family Reference Manual, Sect. 17 10-Bit A/D Converter," September 2009. [Online]. Available: http://ww1.microchip.com/downloads/en/DeviceDoc/39705b.pdf.

[16] J. Liu, "09.1 UART Setup," 16 April 2008. [Online]. Available: http://www.engscope.com/pic24-tutorial/9-1-uart-setup/.

[17] TDK-Lambda Corporation, "JWS240P Evaluation Data," [Online]. Available: http://www.tdk-lambda.com/products/sps/ps_unit/jws_p/pdf/jws240p_eva.pdf. 


\section{Appendix A: Additional Hardware Design Information}

Table A-1 and Table A-2 show calculation results for the voltage sense resistor dividers.

Table A-1shows the set parameters, while Table A-2 shows the results of calculations to determine the resistor values, as well as the filter capacitors used. The switching frequency for $\mathrm{V}_{\text {IN }}$ was taken from the power supply evaluation datasheet [17] and the switching frequency for $\mathrm{V}_{\text {OUT }}$ was taken from the DC-DC converter datasheet [10].

Table A-1. Input and Output Voltage Ranges

\begin{tabular}{cccccc}
\hline Parameter & Symbol & Minimum & Typical & Maximum & Units \\
\hline Input Voltage & $\mathrm{V}_{\mathrm{IN}}$ & 46 & 48 & 50 & $\mathrm{~V}$ \\
Output Voltage & $\mathrm{V}_{\text {OUT }}$ & 4.85 & 5 & 5.15 & V \\
Desired Set Voltage & $\mathrm{V}_{\text {SET }}$ & - & 3 & - & $\mathrm{V}$ \\
\hline
\end{tabular}

Table A-2. Calculation Results for Input and Output Voltage Sense Resistor Dividers

\begin{tabular}{cccc}
\hline Parameter & $\mathbf{V}_{\text {IN }}$ & V $_{\text {OUT }}$ & Units \\
\hline Desired Current & 0.5 & 0.5 & $\mathrm{~mA}$ \\
Actual Current & 0.504 & 0.498 & $\mathrm{~mA}$ \\
R1+R2 Desired & 100000 & 10300 & $\Omega$ \\
R1 Desired & 94000 & 4300 & $\Omega$ \\
R2 Desired & 6000 & 6000 & $\Omega$ \\
R1 Actual & 93100 & 4300 & $\Omega$ \\
R2 Actual & 6040 & 6040 & $\Omega$ \\
Max. sense voltage & 3.05 & 3.01 & $\mathrm{~V}$ \\
Sense at $\mathrm{V}_{\text {TYP }}$ & 2.924 & 2.921 & $\mathrm{~V}$ \\
Sense Ratio & 0.061 & 0.584 & - \\
Parallel resistance R & 5672 & 2512 & $\Omega$ \\
Switching Frequency $\mathrm{F}_{\mathrm{SW}}$ & 297 & 290 & $\mathrm{kHz}$ \\
Filter Capacitor Desired & 270 & 624 & $\mathrm{pF}$ \\
Filter Capacitor Actual & 270 & 620 & $\mathrm{pF}$ \\
\hline
\end{tabular}


Table A-3 and Table A-4 below show the LOC110 characteristics and support circuitry calculation results, respectively. The information in Table A-3 was taken from the LOC110 datasheet [8] and the calculations used for the results in Table A-4 were taken from the LOC110 Application Note [9].

Table A-3. LOC110 Device Characteristics

\begin{tabular}{cccccc}
\hline Parameter & Symbol & Minimum & Typical & Maximum & Units \\
\hline LED Voltage Drop & $\mathrm{V}_{\mathrm{F}}$ & 0.9 & 1.2 & 1.4 & $\mathrm{~V}$ \\
K1, Servo Gain $\left(\mathrm{I}_{\mathrm{C} 1} / \mathrm{I}_{\mathrm{F}}\right)$ & $\mathrm{K} 1$ & 0.004 & 0.007 & 0.03 & - \\
$\mathrm{K} 2$, Forward Gain $\left(\mathrm{I}_{\mathrm{C} 2} / \mathrm{I}_{\mathrm{F}}\right)$ & $\mathrm{K} 2$ & 0.004 & 0.007 & 0.03 & - \\
$\mathrm{K} 3$, Transfer Gain $\left(\mathrm{K} 2 / \mathrm{K} 1=\mathrm{I}_{\mathrm{C} 2} / \mathrm{I}_{\mathrm{C} 1}\right)$ & $\mathrm{K} 3$ & 0.668 & 1 & 1.179 & - \\
Input-Side Supply Voltage & $\mathrm{V}_{\mathrm{CC} 1}$ & 5 & 5 & 5 & $\mathrm{~V}$ \\
Output-Side Supply Voltage & $\mathrm{V}_{\mathrm{CC} 2}$ & 3.267 & 3.3 & 3.333 & $\mathrm{~V}$ \\
\hline
\end{tabular}

Table A-4. Calculation Results for LOC110 Support Circuitry

\begin{tabular}{cccccc}
\hline Parameter & Symbol & Minimum & Typical & Maximum & Units \\
\hline Input Voltage Span & $\mathrm{V}_{\mathrm{IN}-S P A N}$ & 0 & - & 3 & $\mathrm{~V}$ \\
Output Voltage Span & $\mathrm{V}_{\text {OUT-SPAN }}$ & 0 & - & 3 & $\mathrm{~V}$ \\
LED Current & $\mathrm{I}_{\mathrm{F}}$ & - & 0.015 & - & $\mathrm{A}$ \\
Input Resistor (Calculated) & $\mathrm{R} 1_{\mathrm{C}}$ & - & 50000 & - & $\Omega$ \\
Output Resistor (Calculated) & $\mathrm{R} 2_{\mathrm{C}}$ & - & 50000 & - & $\Omega$ \\
Input Resistor (Actual) & $\mathrm{R} 1_{\mathrm{A}}$ & - & 50000 & - & $\Omega$ \\
Output Resistor (Actual) & $\mathrm{R} 2_{\mathrm{A}}$ & - & 50000 & - & $\Omega$ \\
LED Resistor (Calculated) & $\mathrm{R}_{\mathrm{FC}}$ & - & 273.3333 & - & $\Omega$ \\
\hline
\end{tabular}

Figure A-1, Figure A-2, and Figure A-3 below show the complete controller board schematic. Likewise, Figure A-4, Figure A-5, and Figure A-6 show the complete power board schematic.

These schematics were created in EAGLE schematic editor. For details on the design shown here, refer to the Hardware Design section above. 


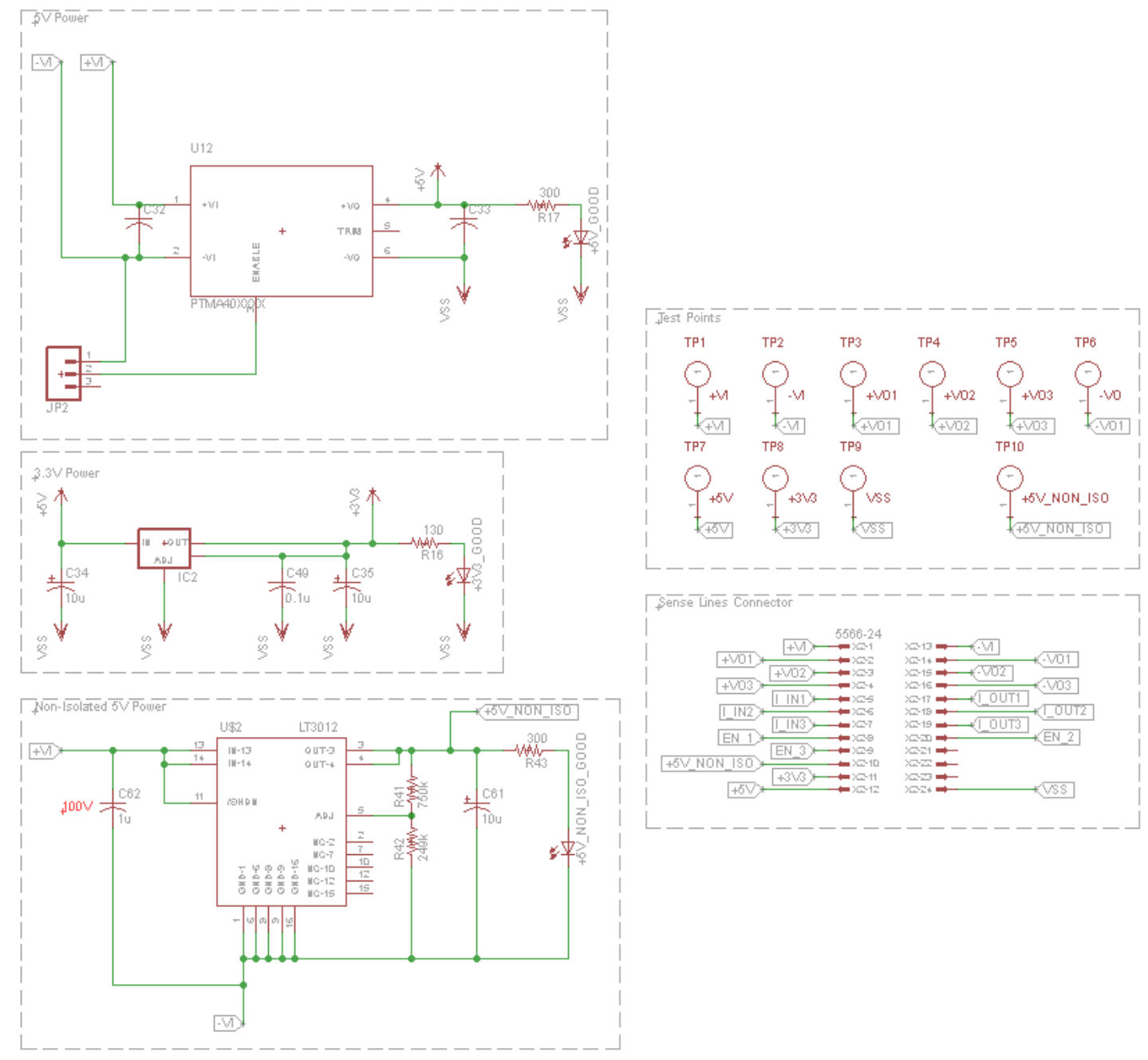

Figure A-1. Controller Schematic-5V, 3.3V, Non-Isolated 5V, Connector, and Test Points 

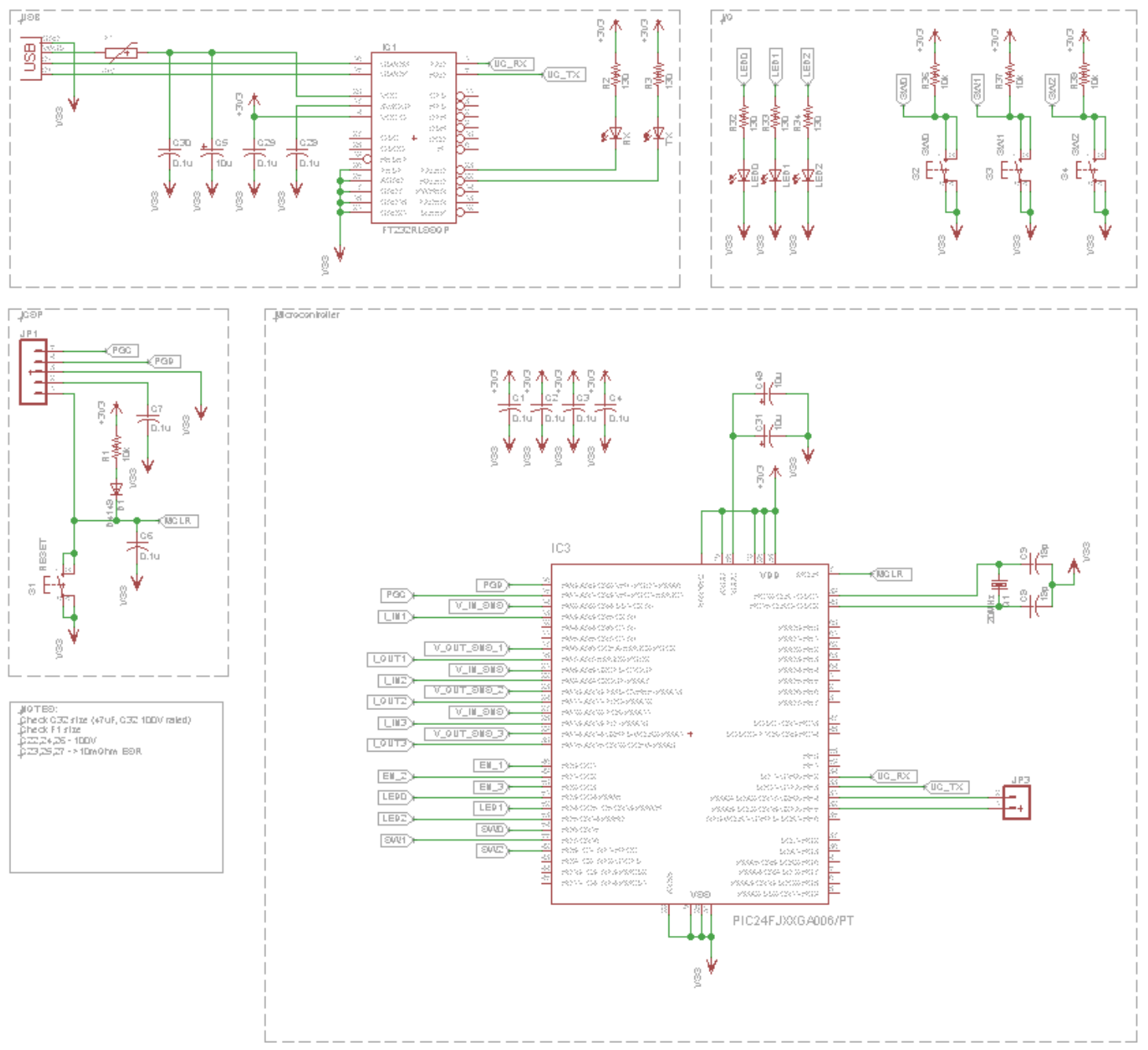

Figure A-2. Controller Schematic - USB, ISCP, Microcontroller, GPIO LEDs and Buttons 


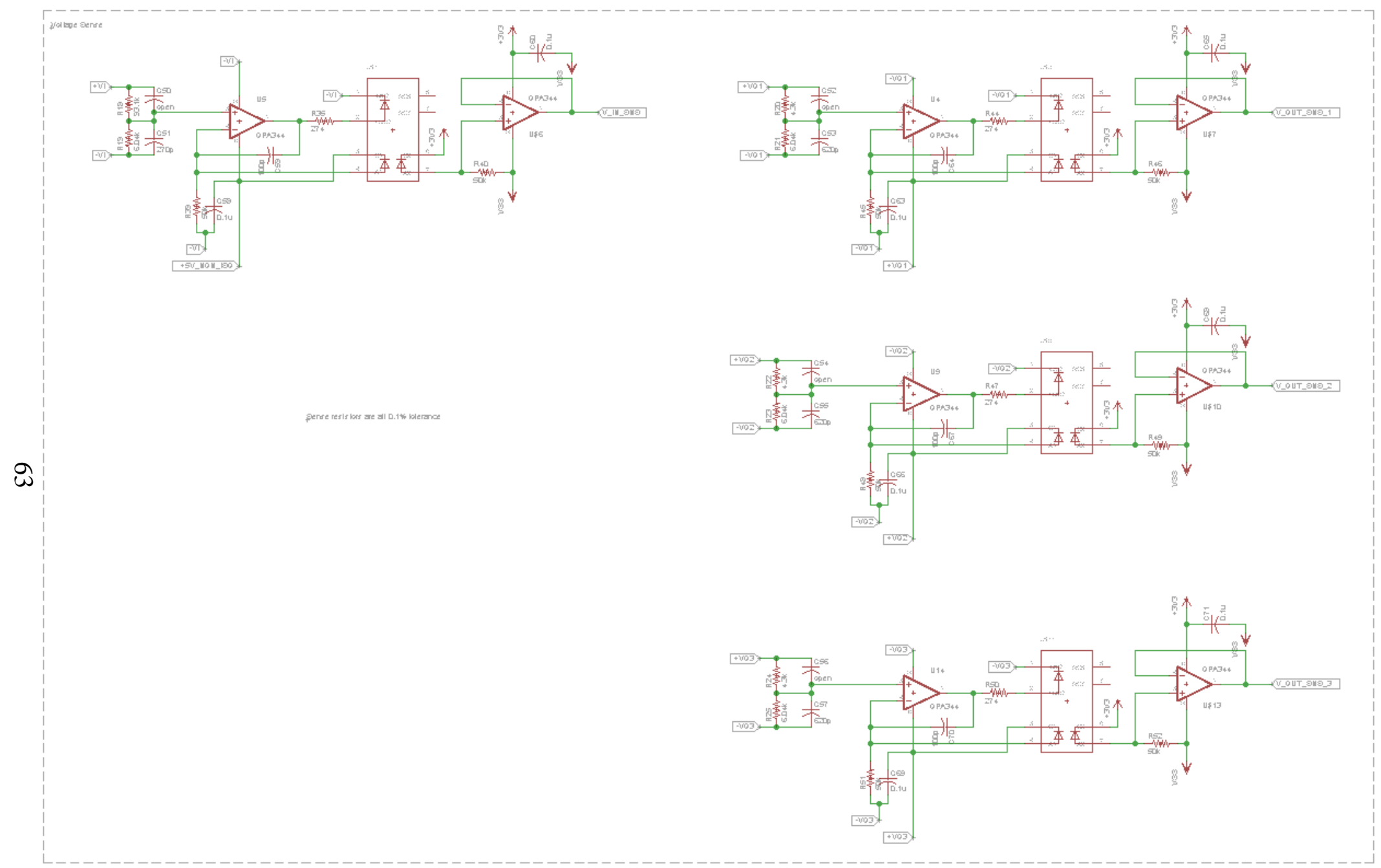

Figure A-3. Controller Schematic - Input and Output Voltage Sense Circuits 


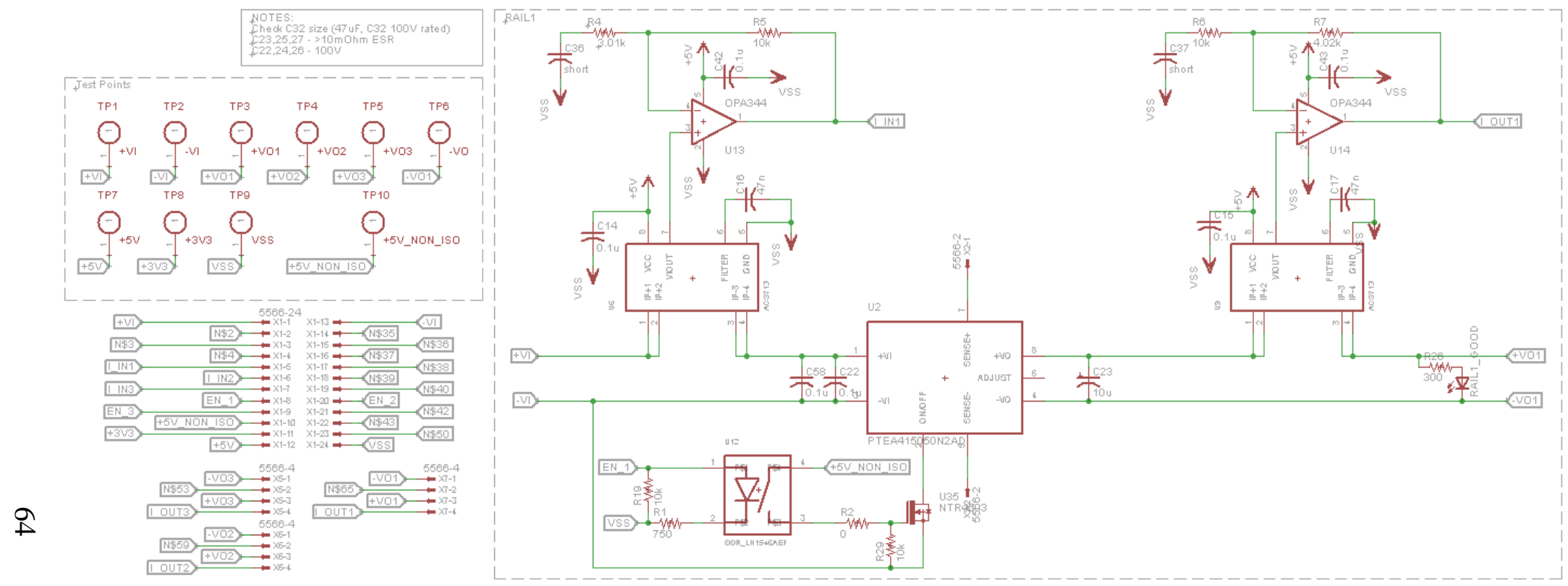

Figure A-4. Power Schematic - Test Points, Connectors, and RAIL 1 


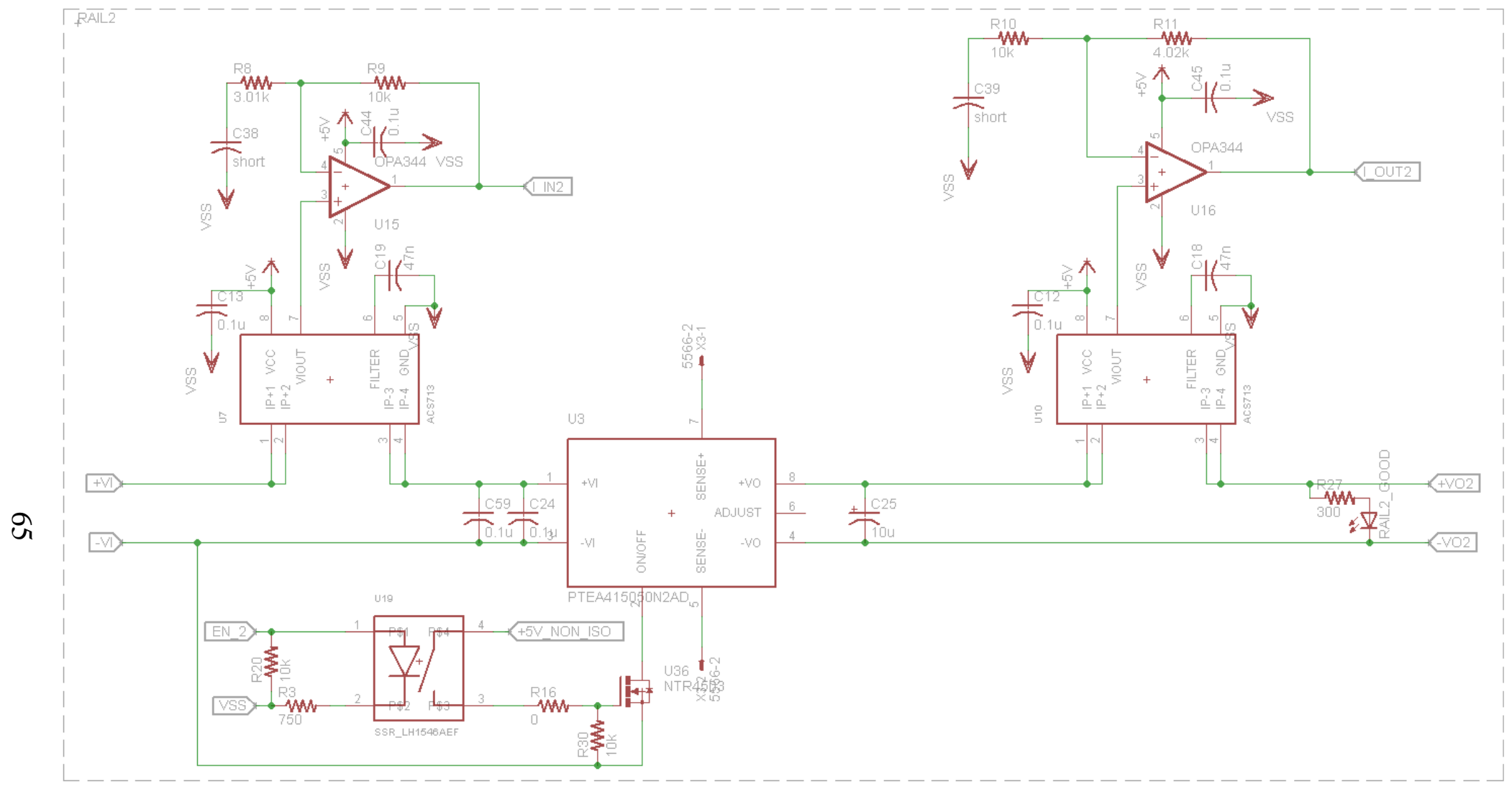

Figure A-5. Power Schematic - RAIL 2 


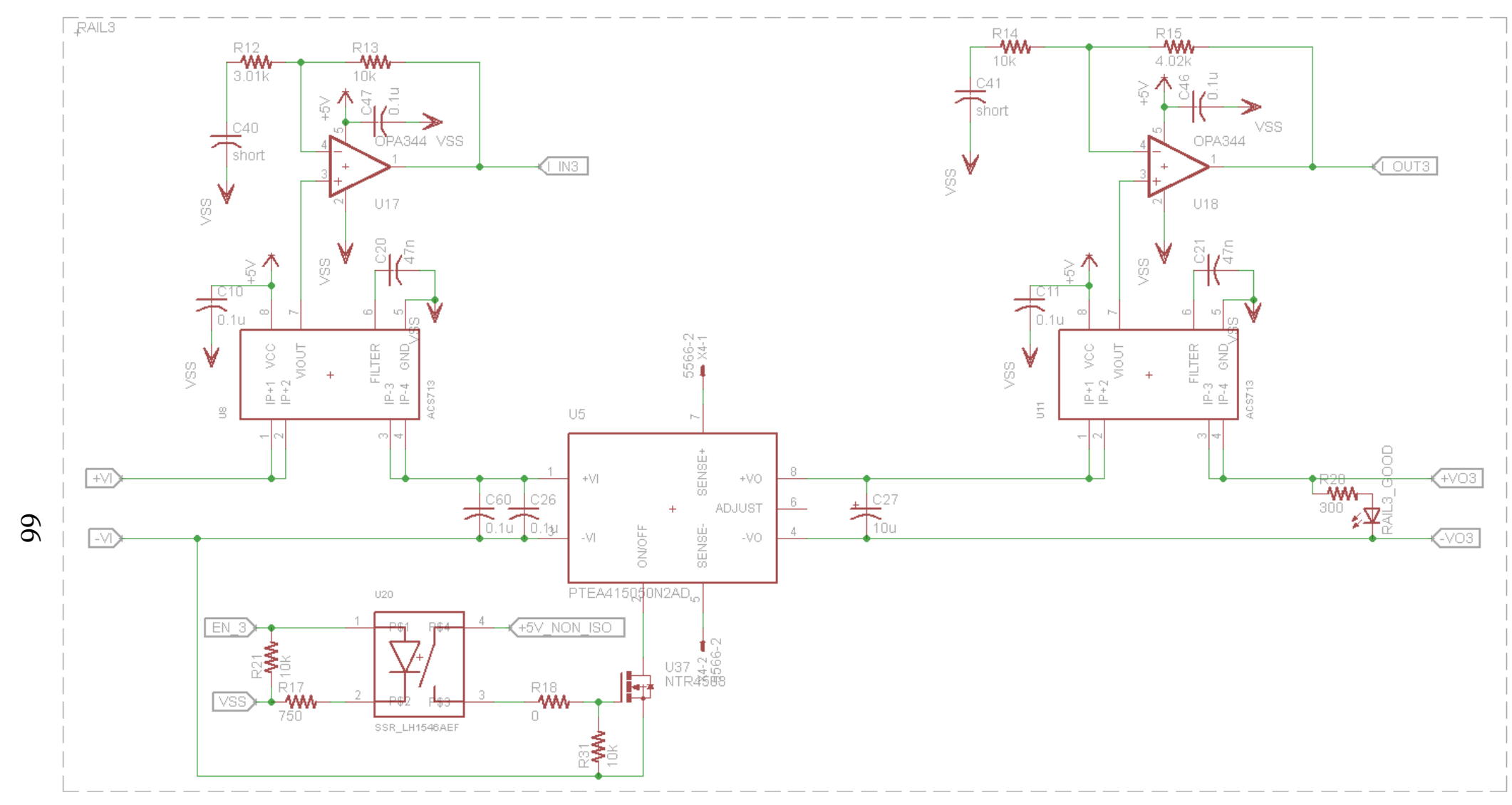

Figure A-6. Power Schematic - RAIL 3 


\section{Appendix B: Simulation Model Code}

Below is a listing of the code used to run the Simulink model discussed in this thesis. See the Simulation section above for an explanation of each file.

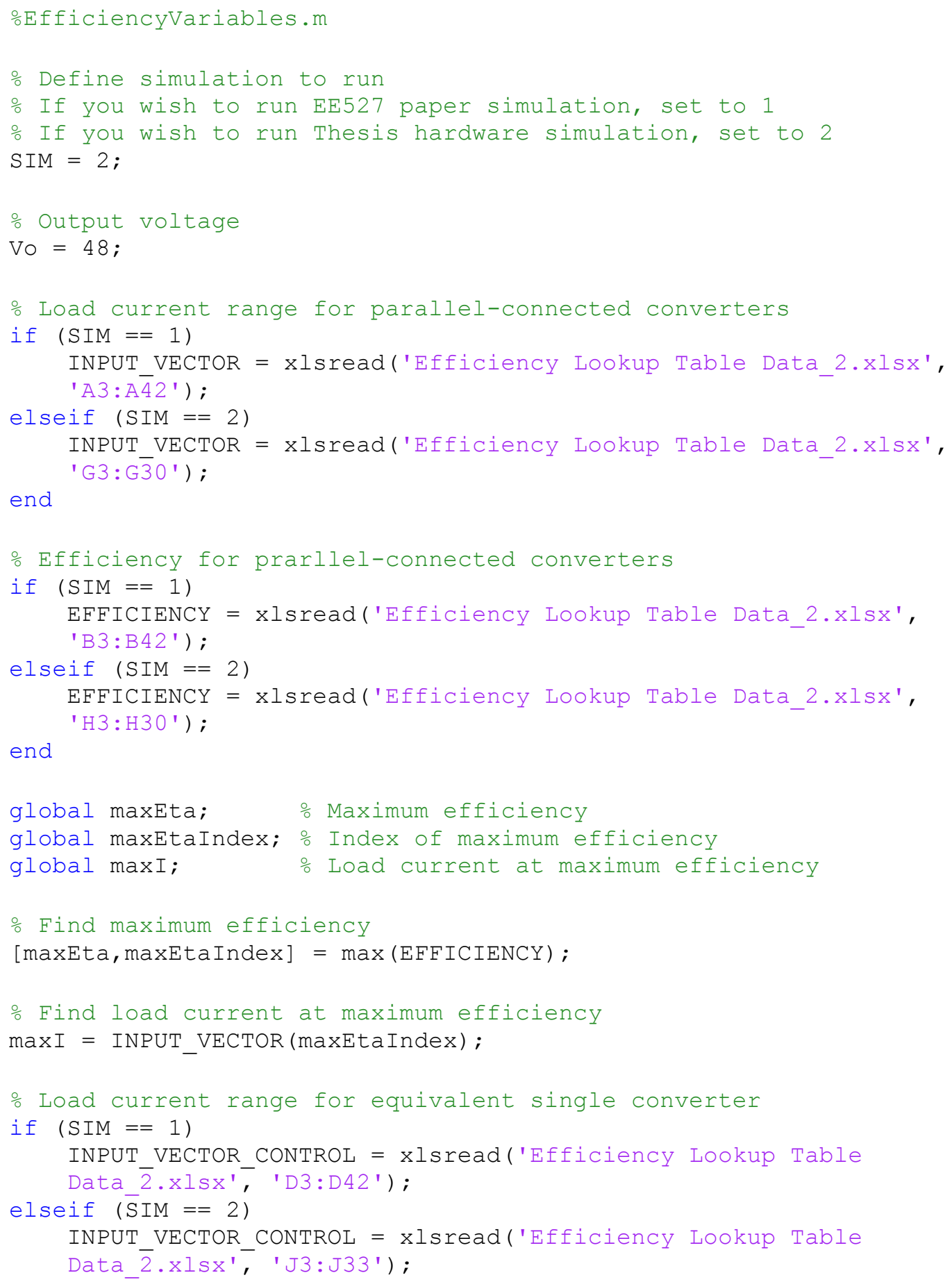




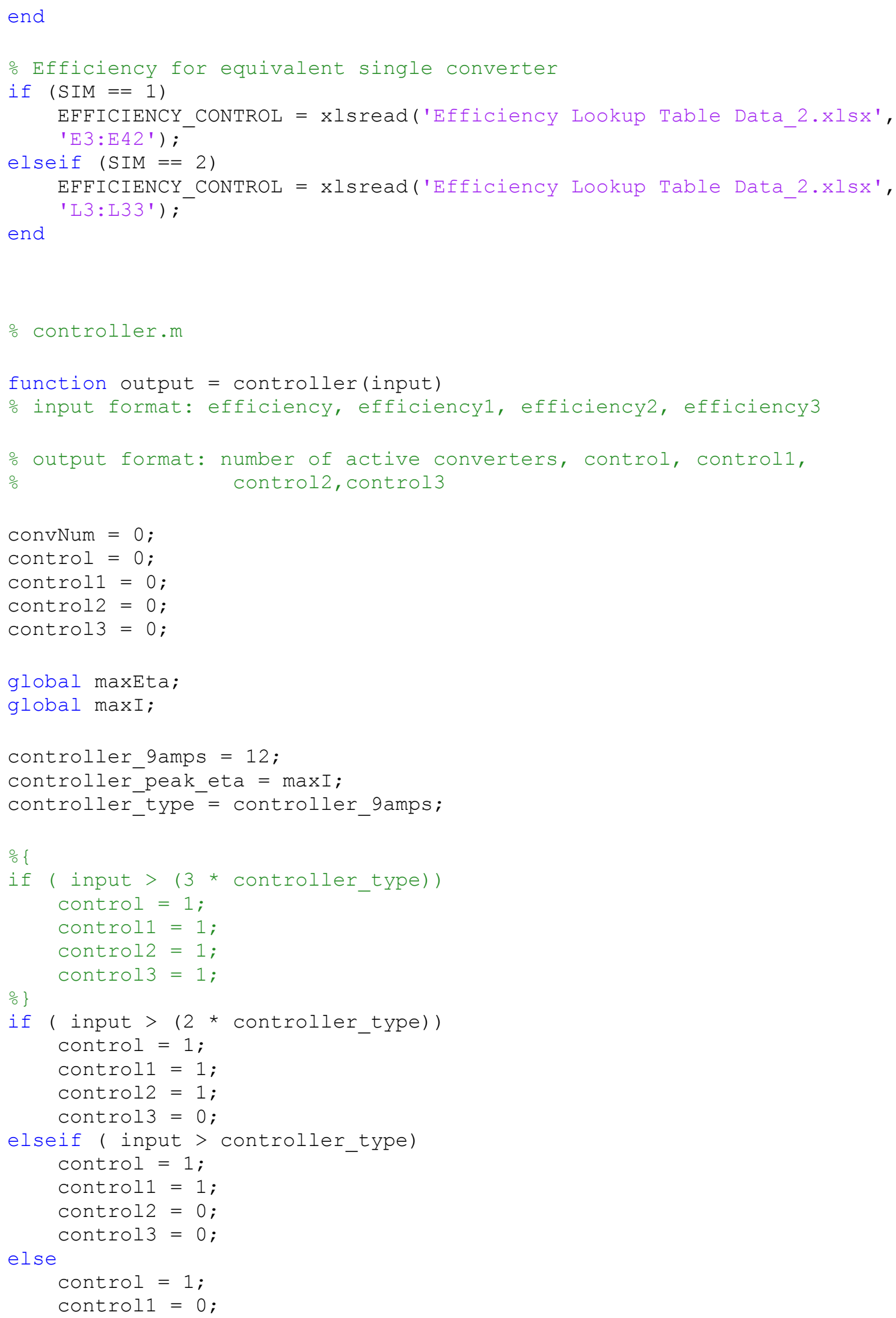




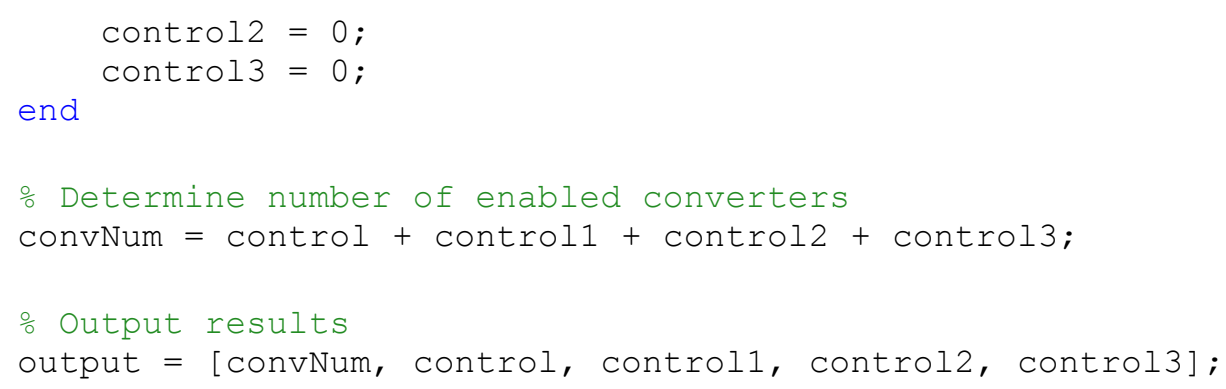




\section{Appendix C: Embedded Firmware Code}

Below is a listing of the firmware code used to run the hardware discussed in this thesis. See the Software Design - Embedded Firmware section above for an explanation of each file. The code is written in $C$ and was compiled using the Microchip C30 compiler in MPLAB IDE.

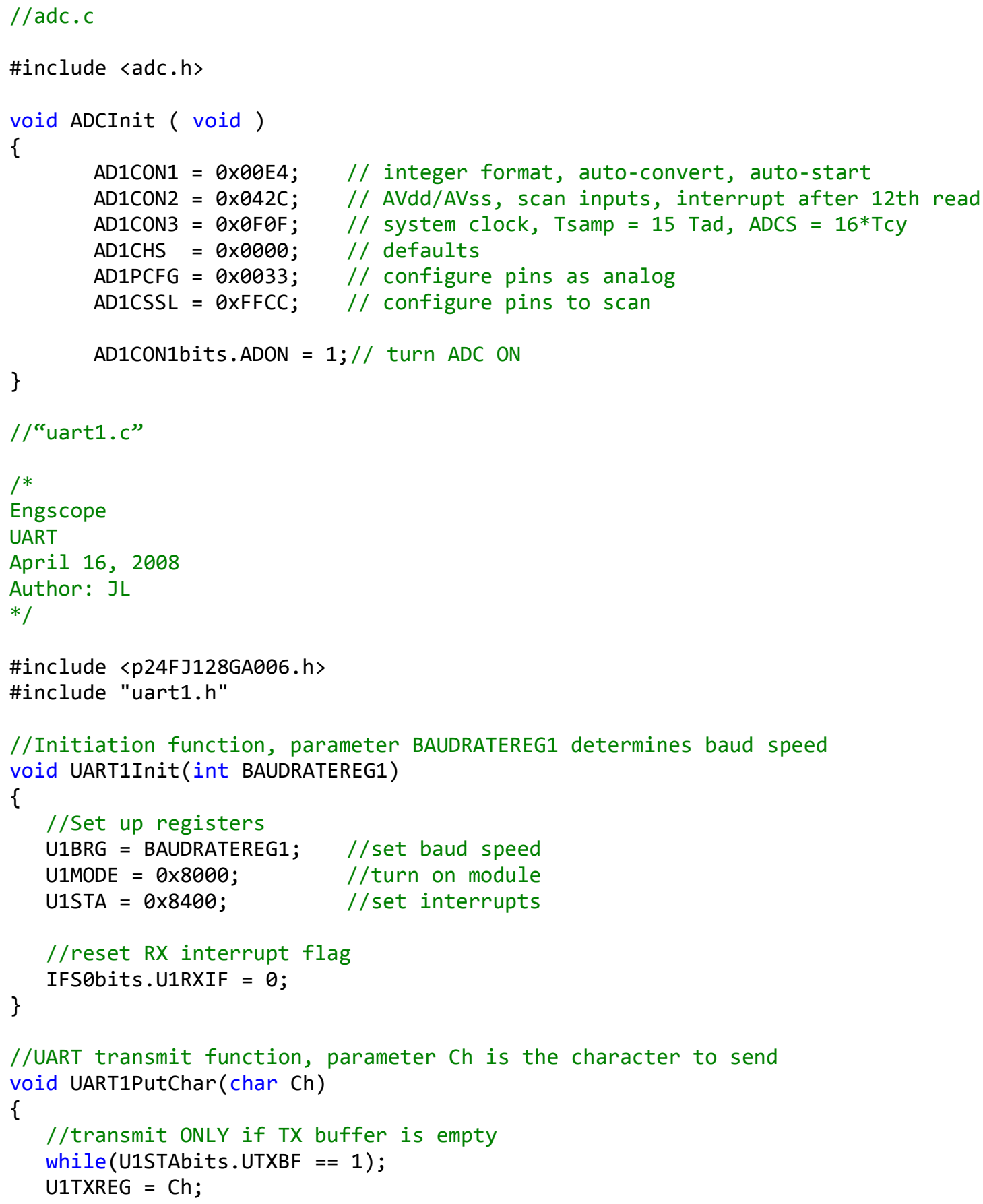




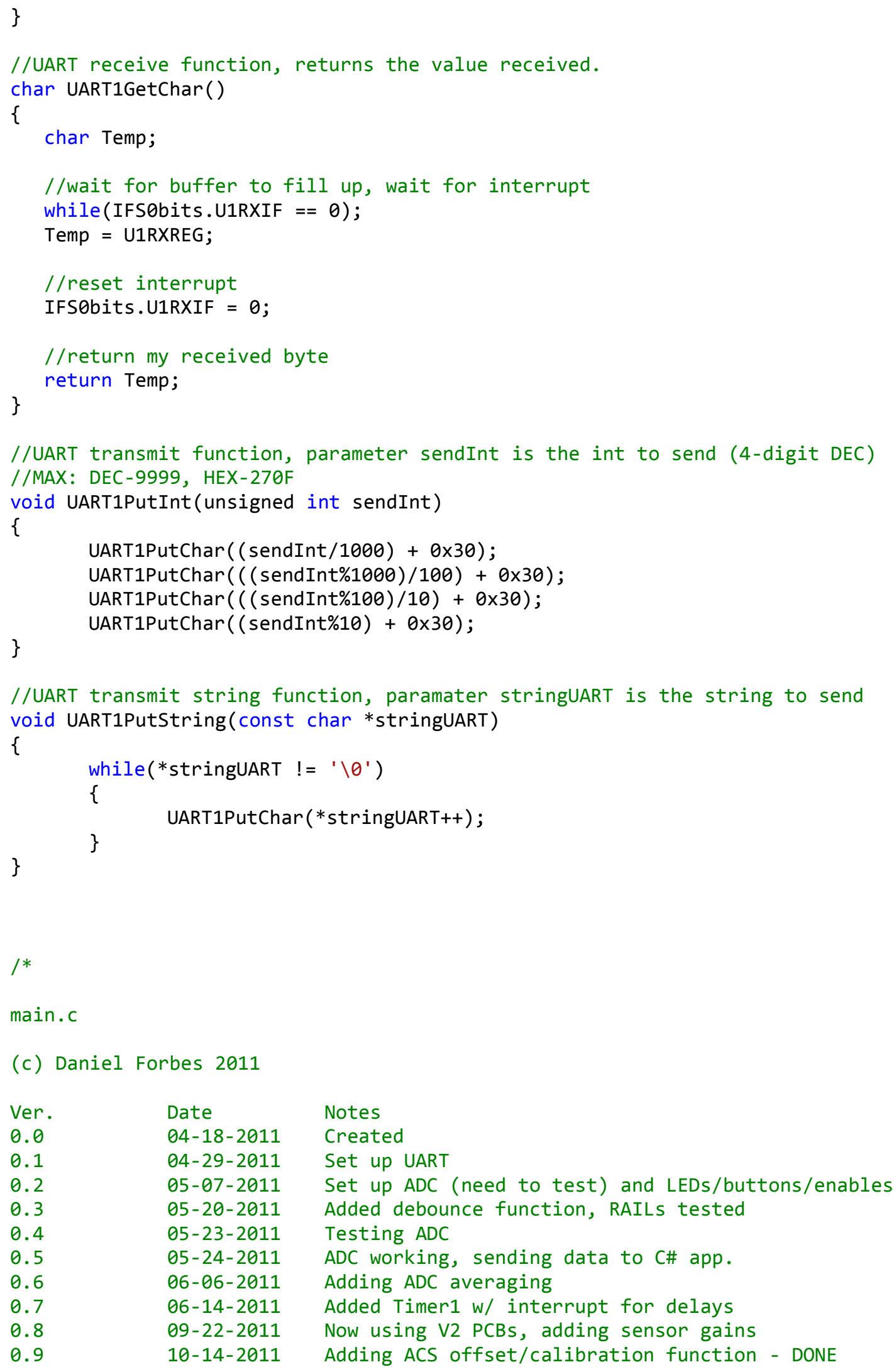




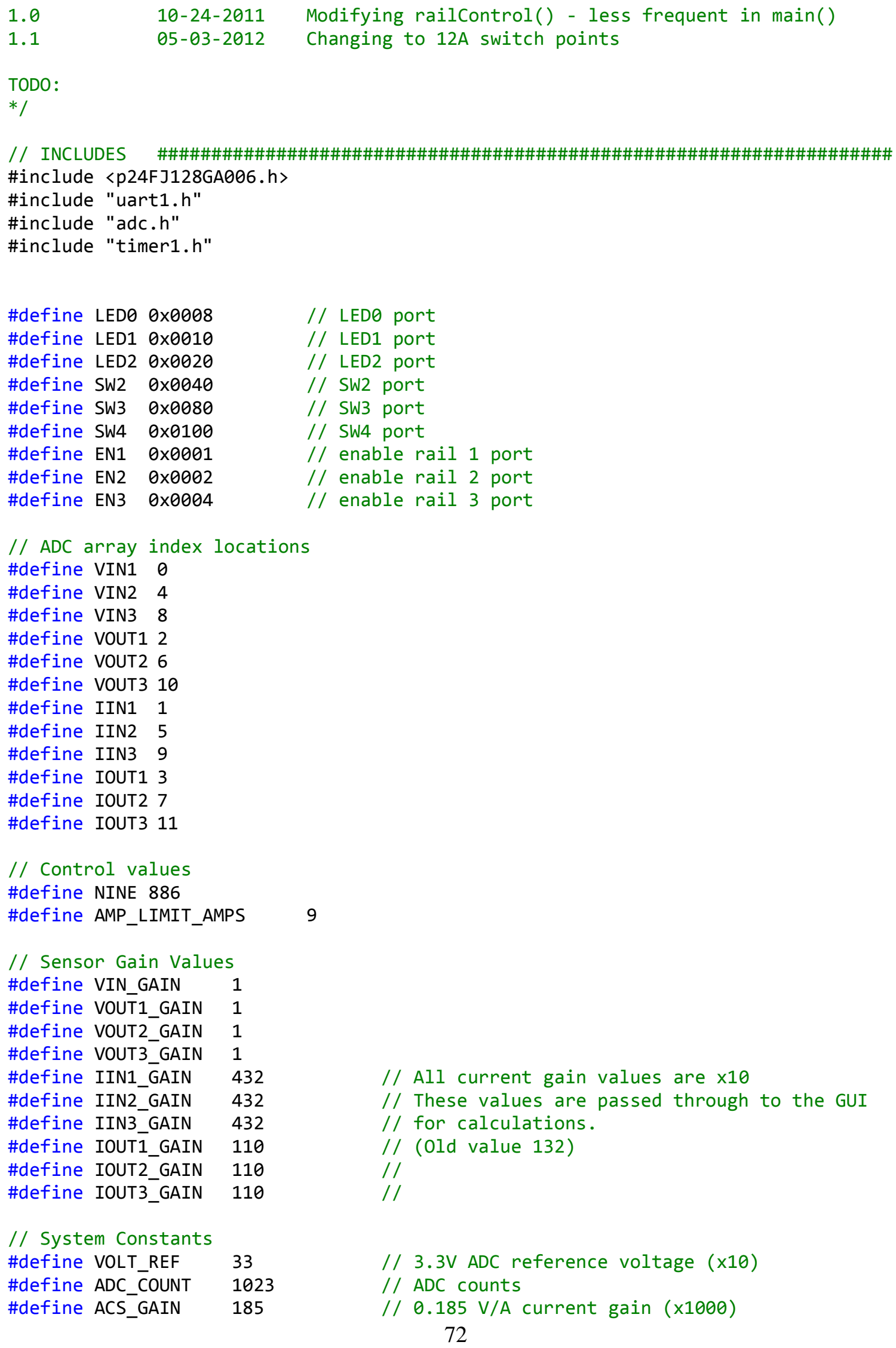




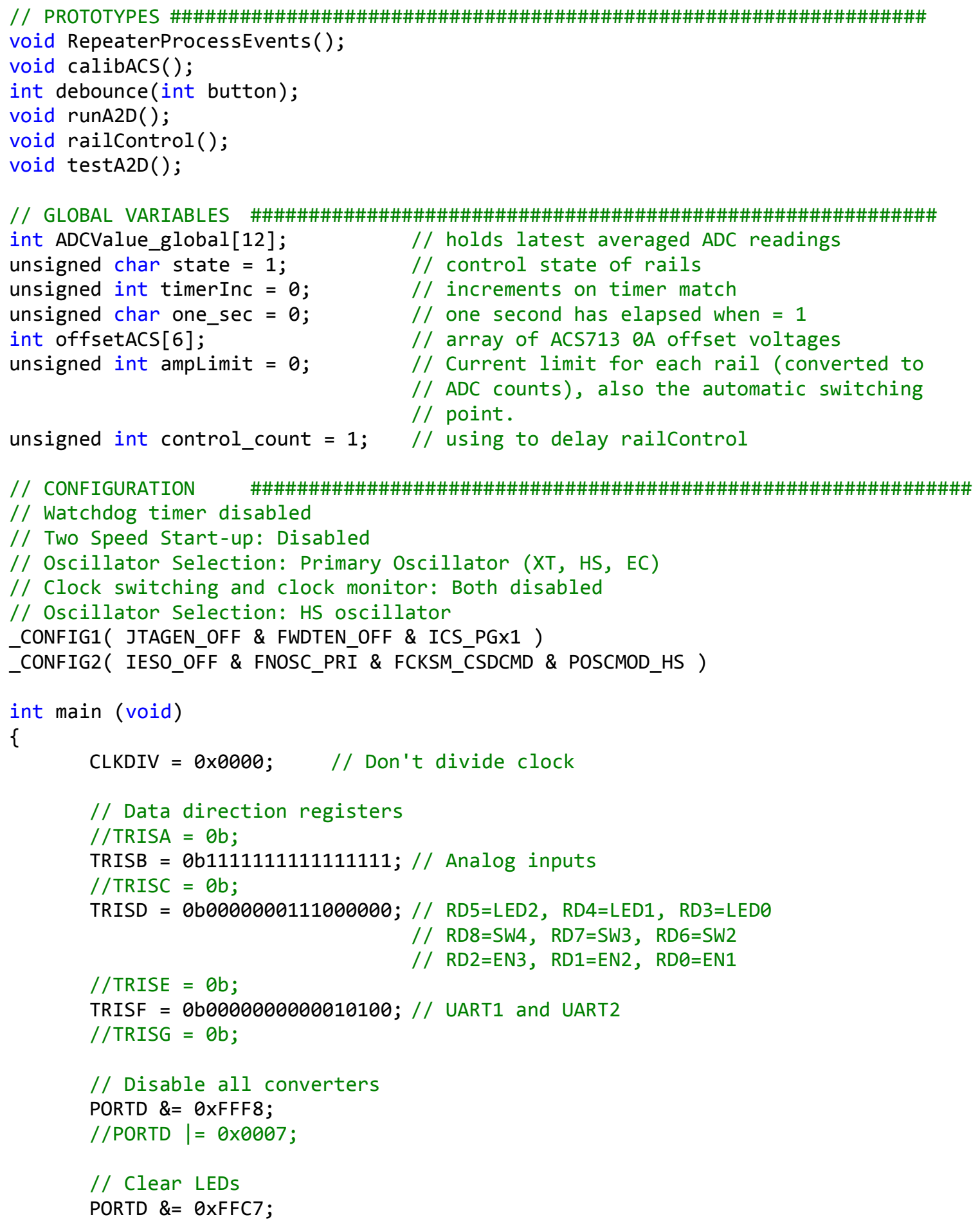

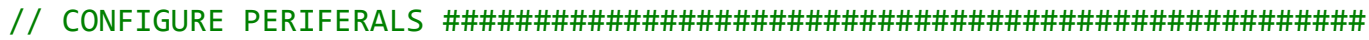
UART1Init(65); ADCInit ( ); // Initiate UART1 to 9600 at 20MHz OSCI

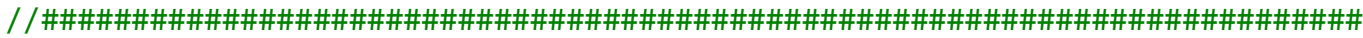
// Read in OA ACS713 voltage offset 


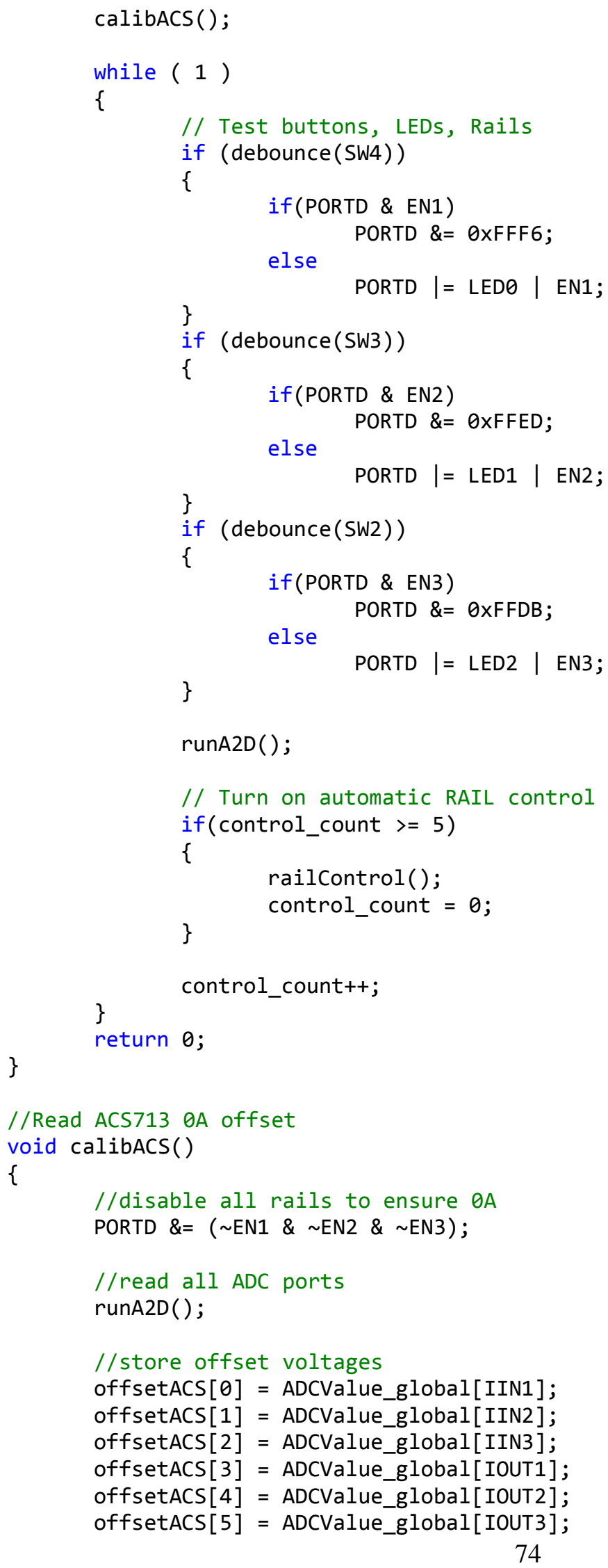




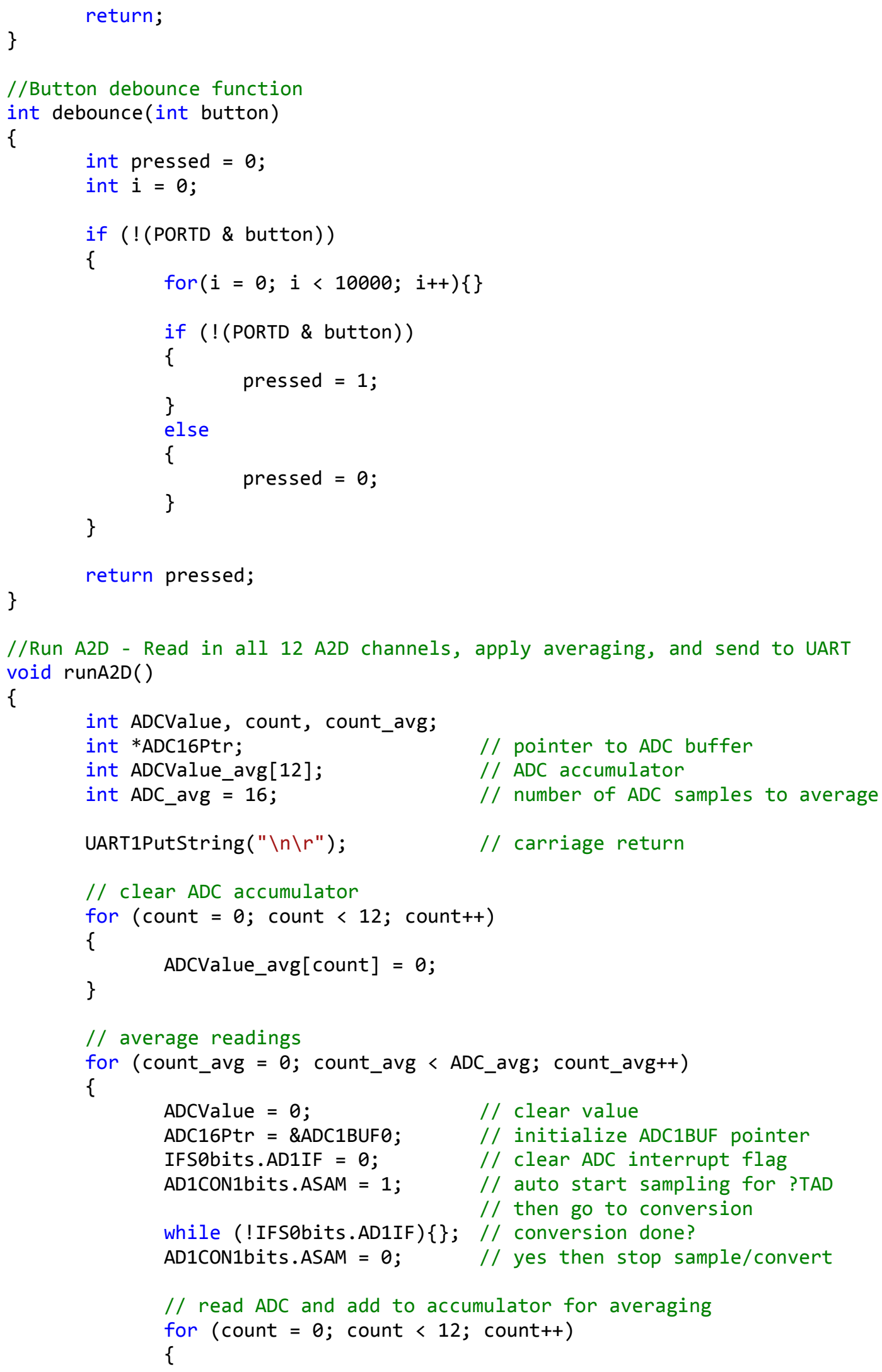




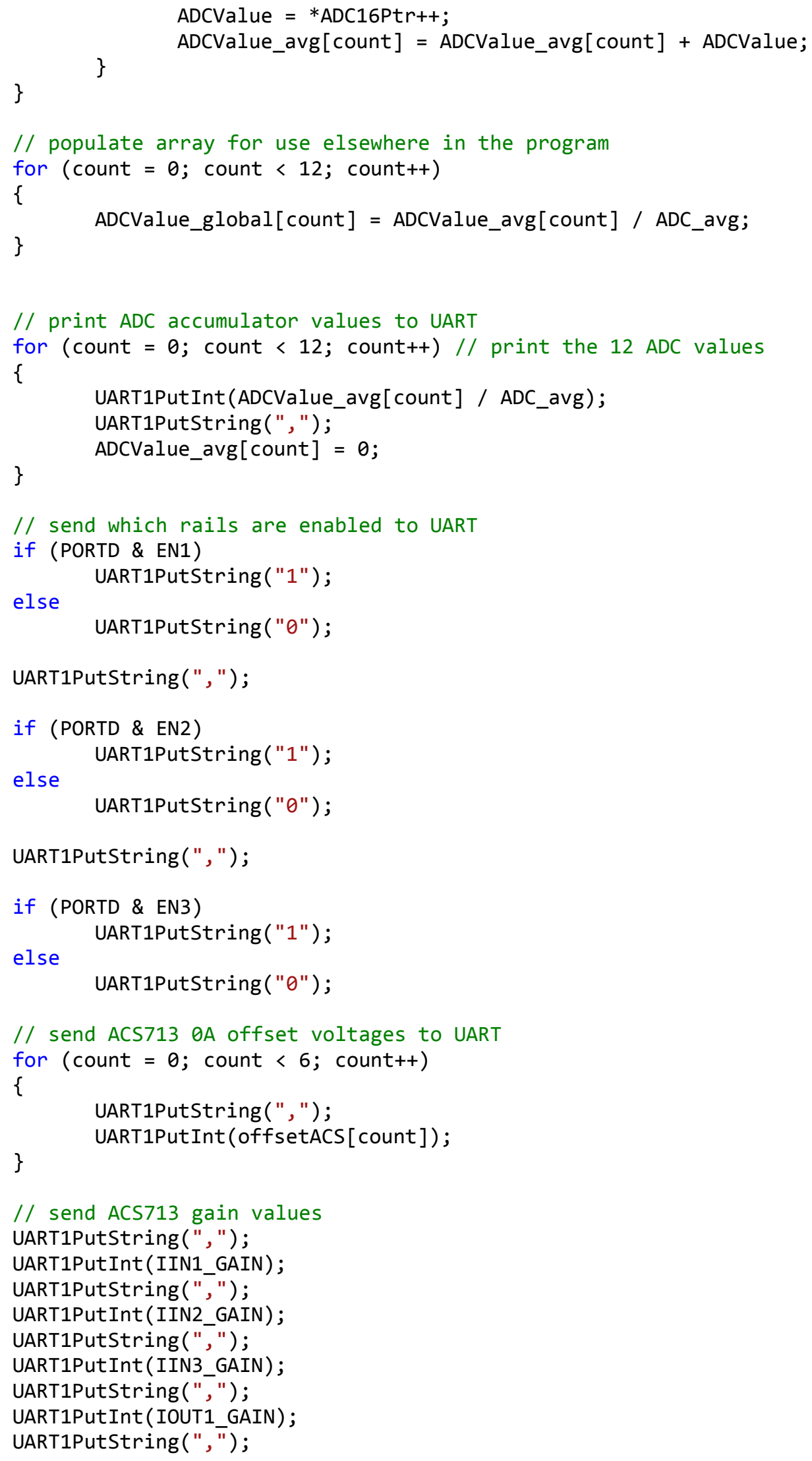




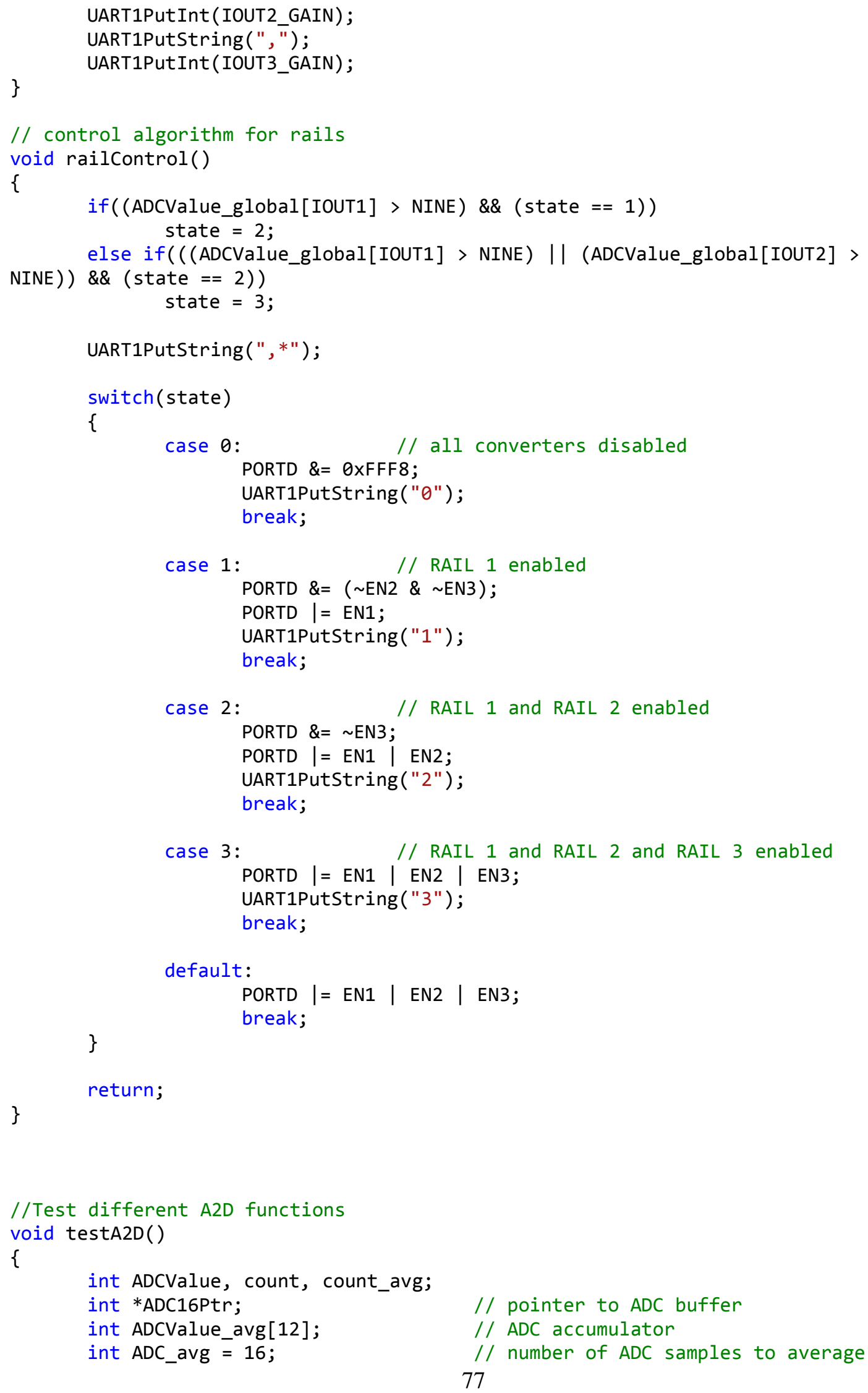




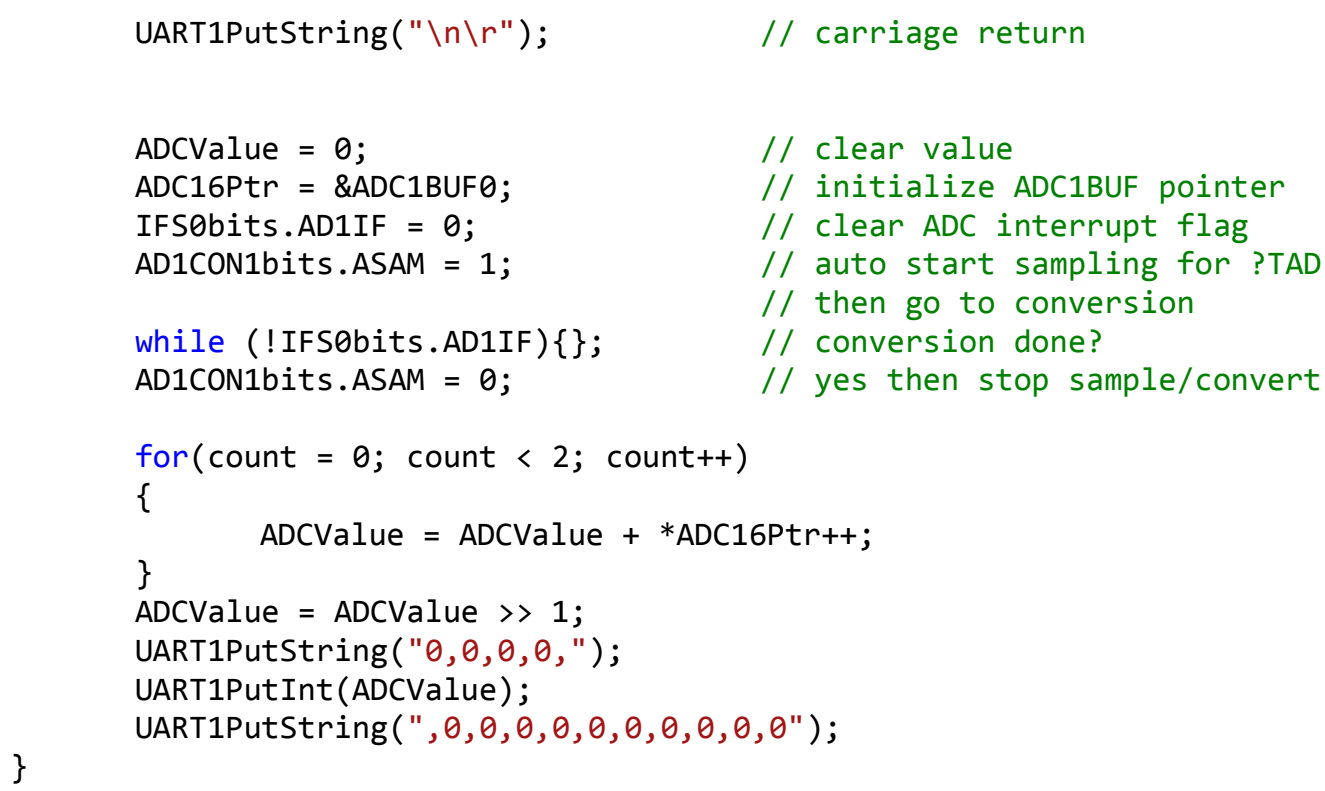




\section{Appendix D: PC Software Code}

Below is a listing of the software code used to run on a PC. See the Software Design - PC Software section above for an explanation of the code. The code is written in $C \#$ and was compiled using Microsoft Visual C\# 2010 Express.

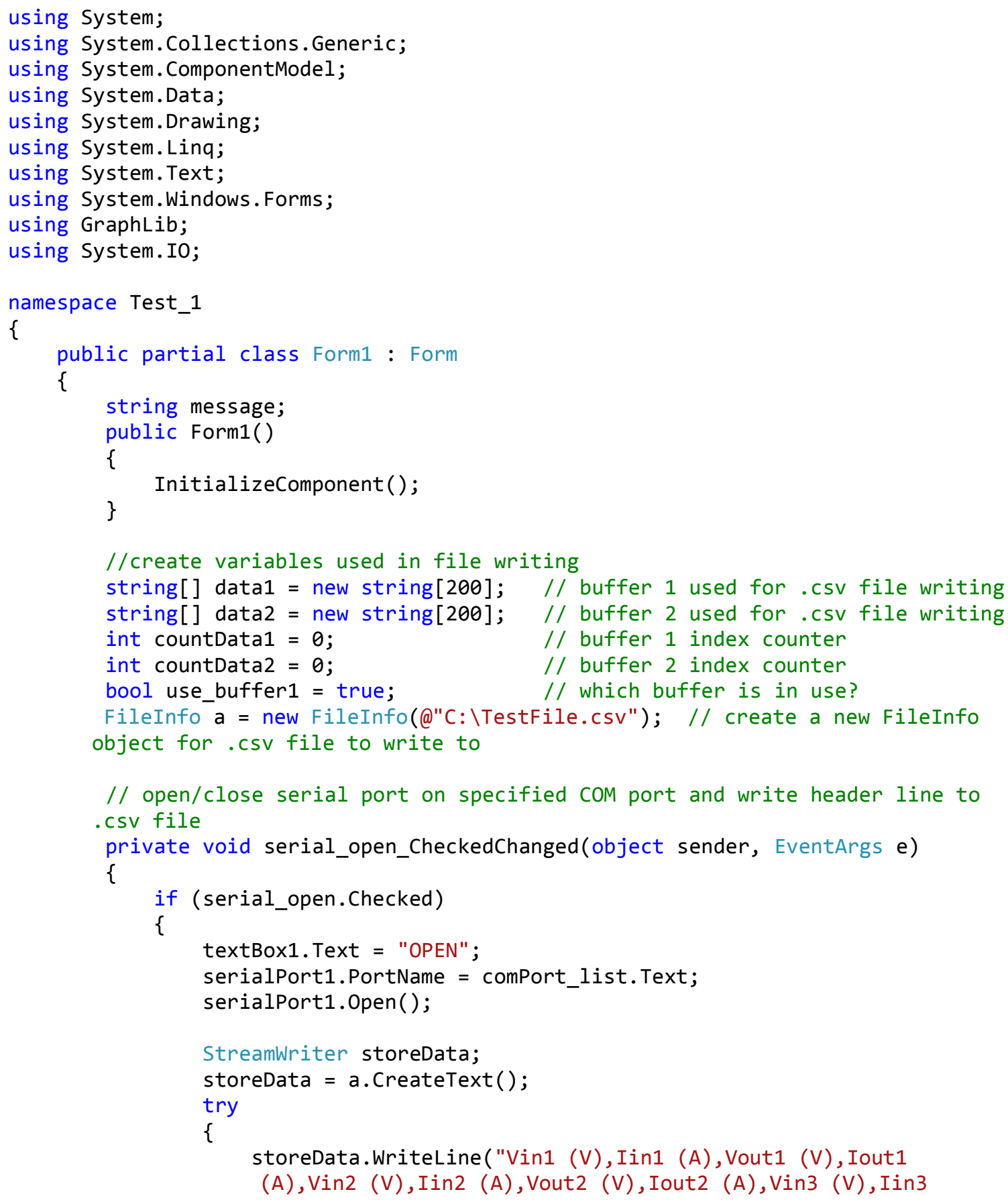




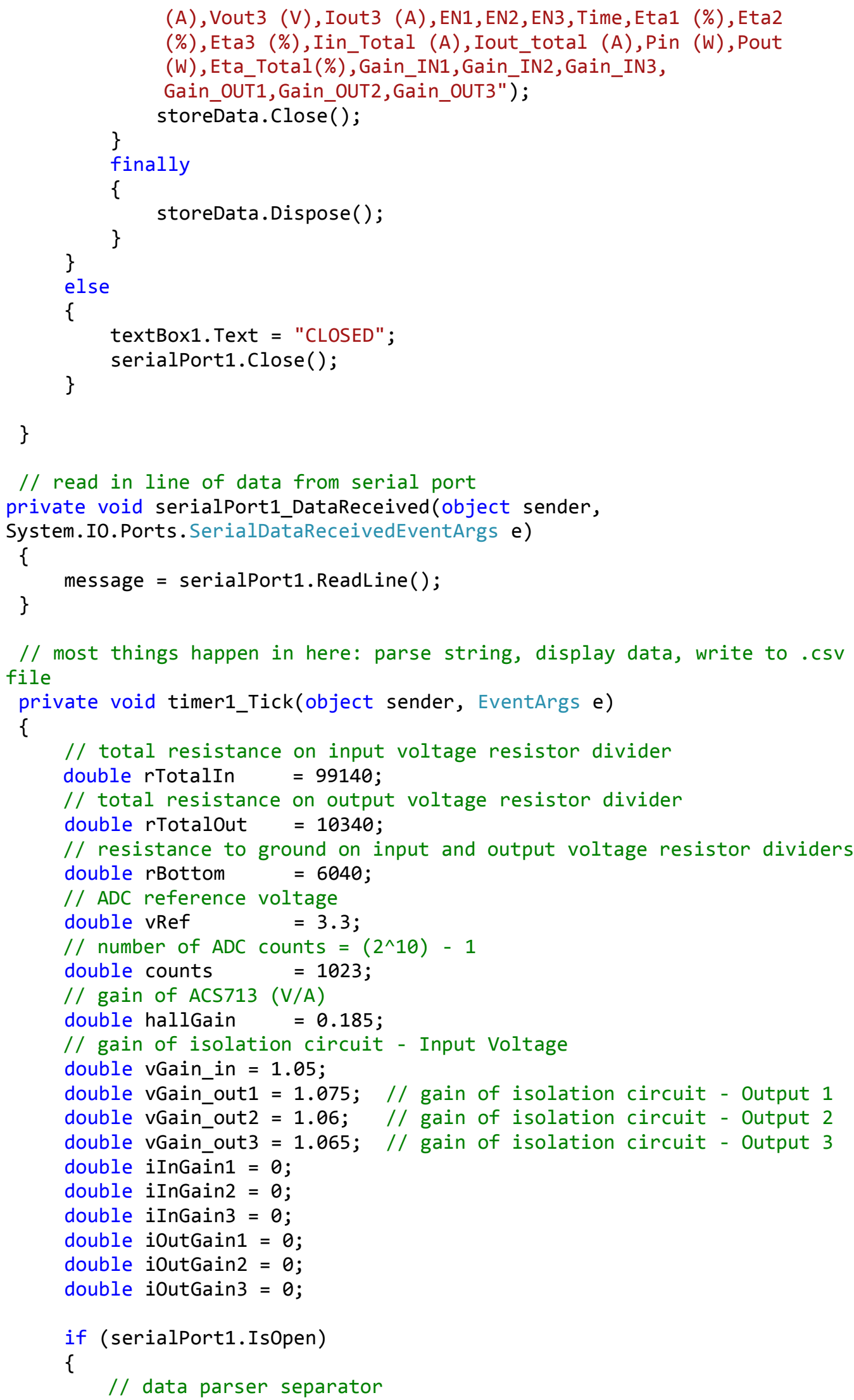




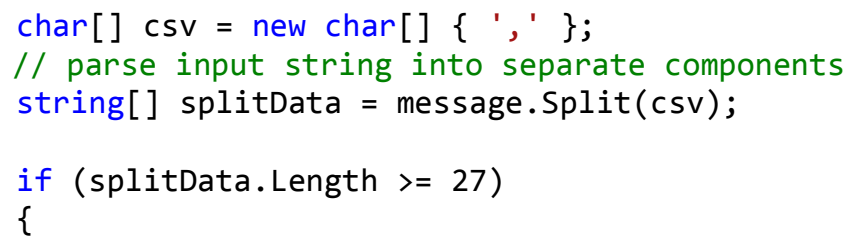

System.Diagnostics. Debug. WriteLine(splitData. Length. ToString( ));

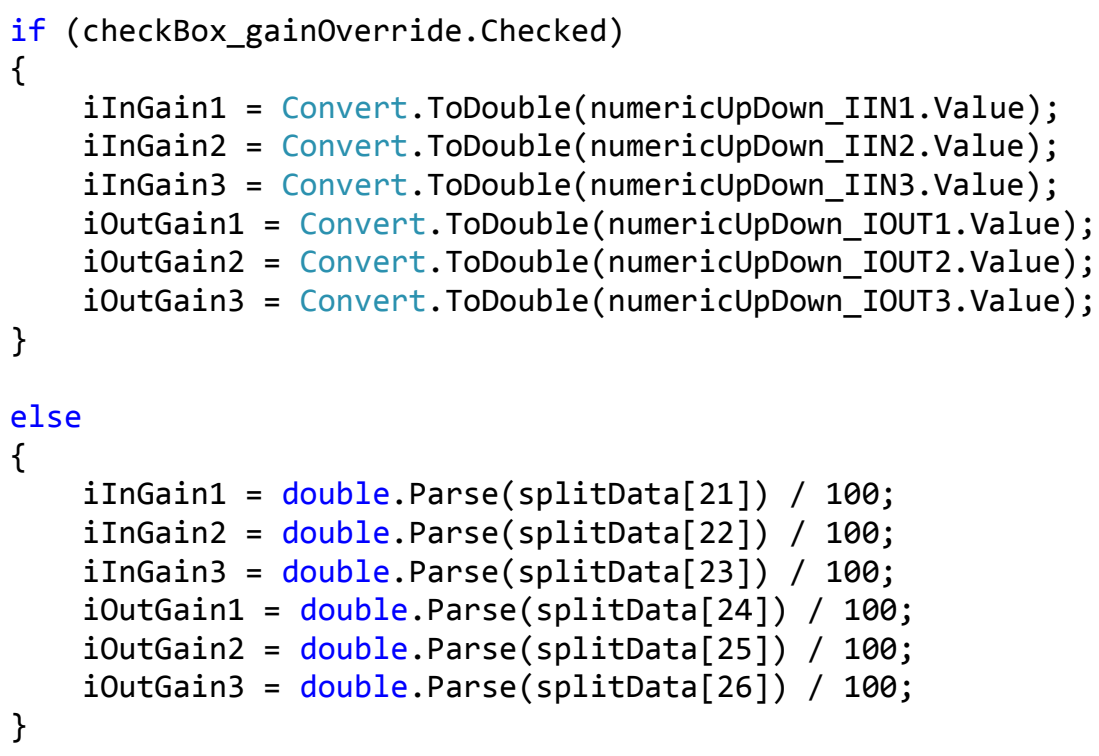




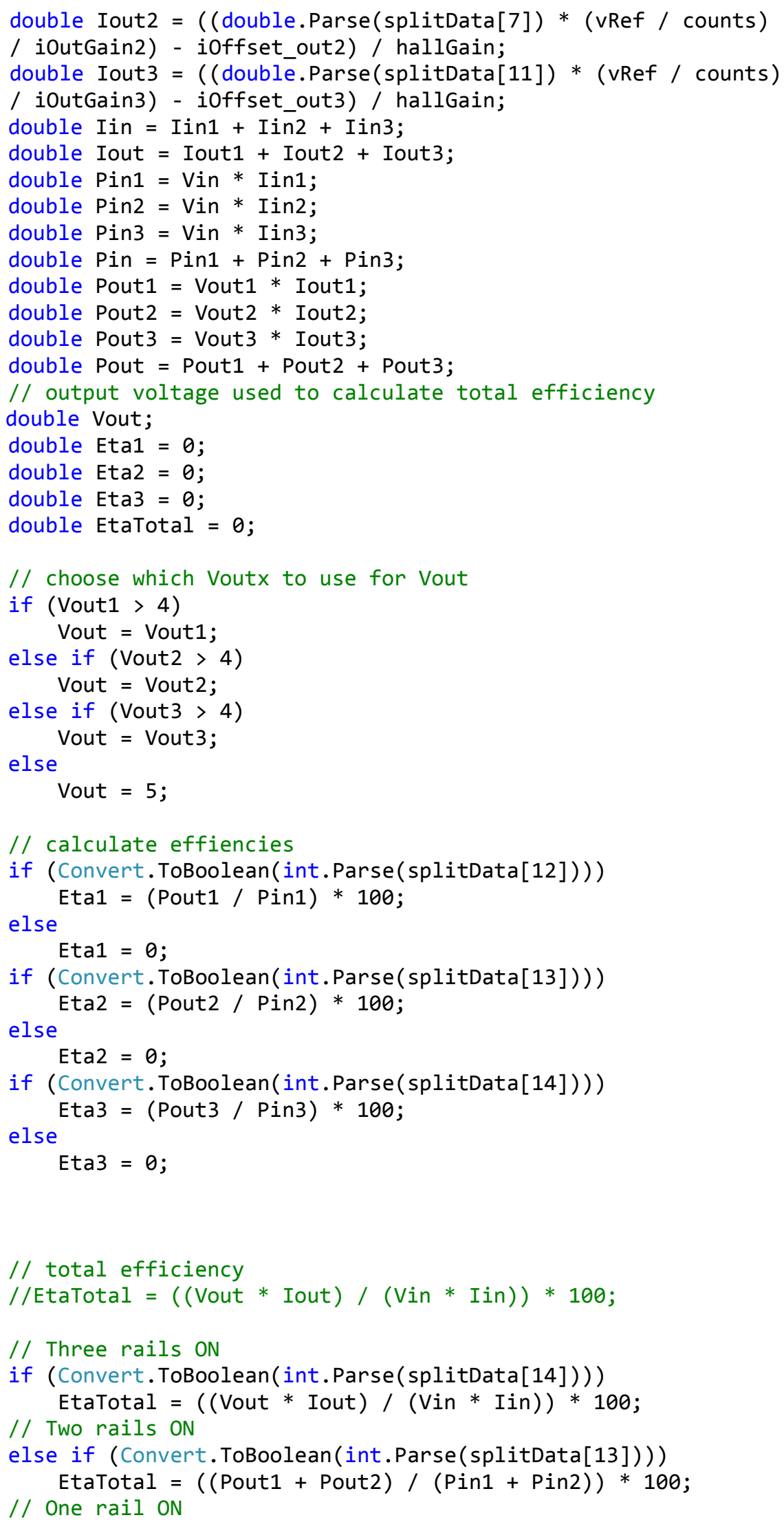




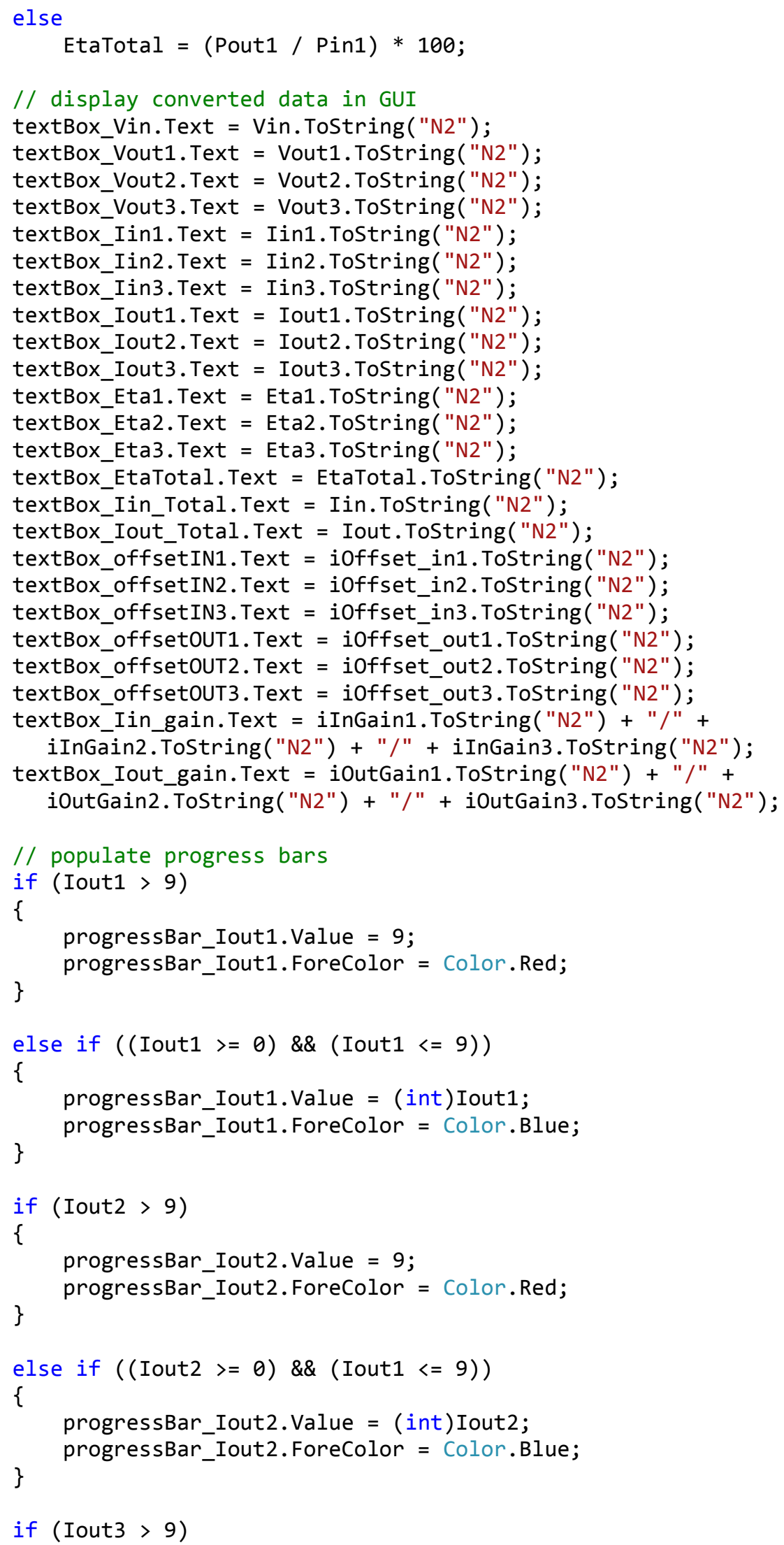




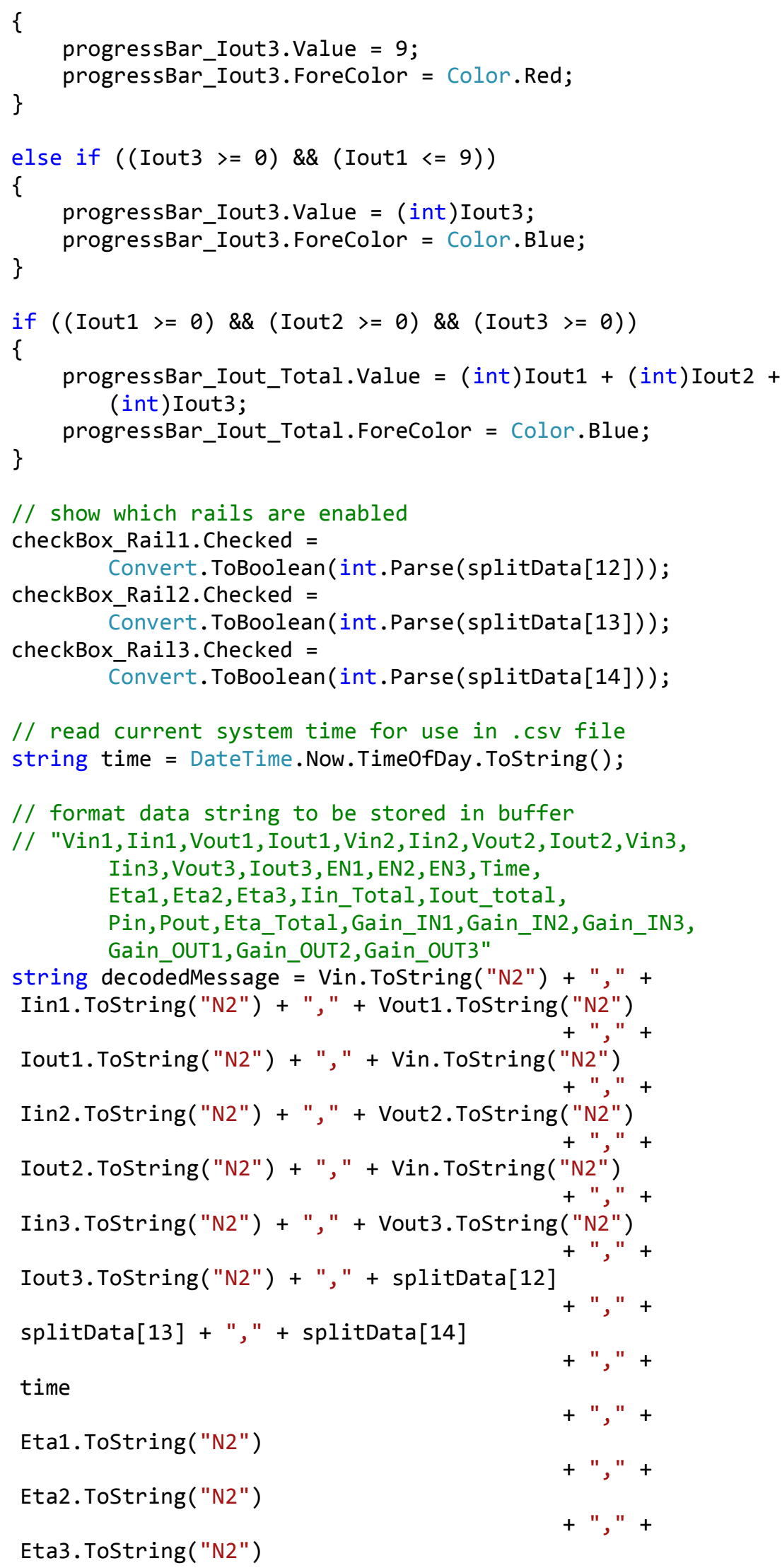




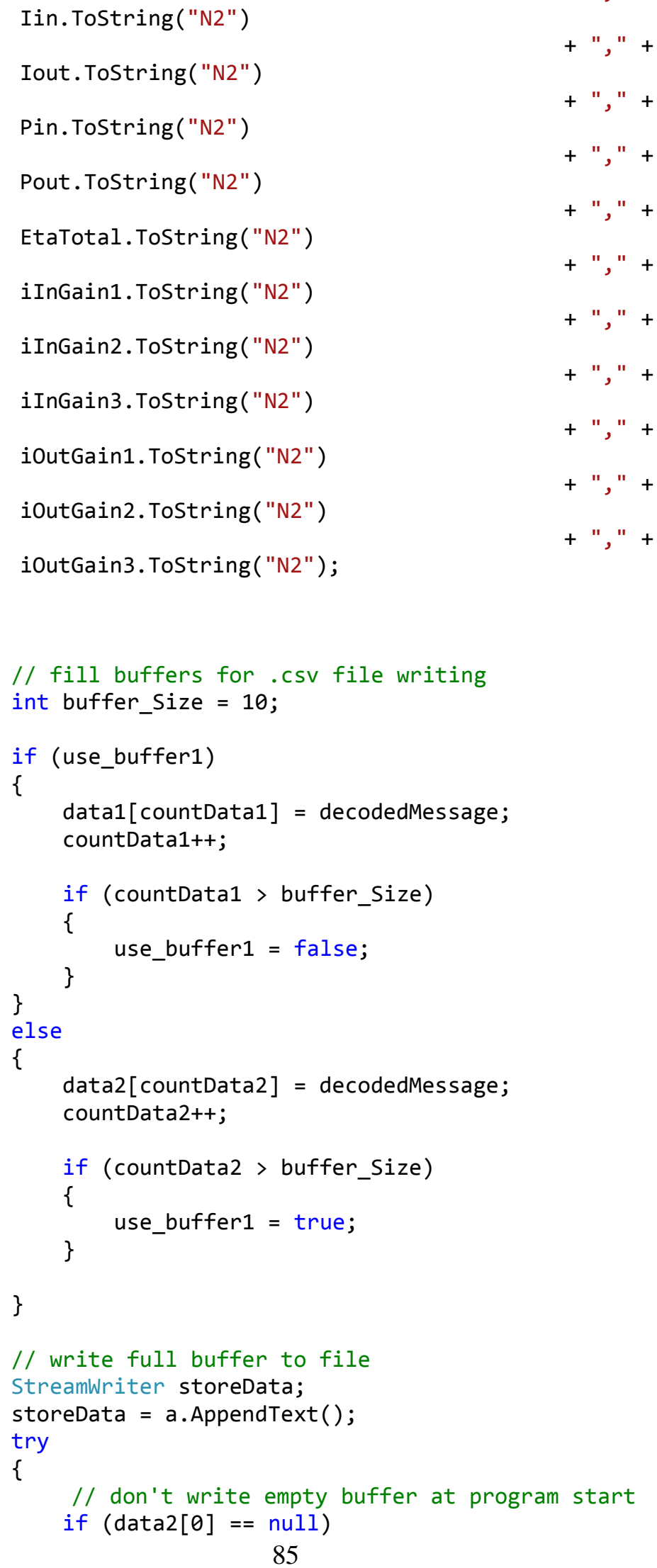




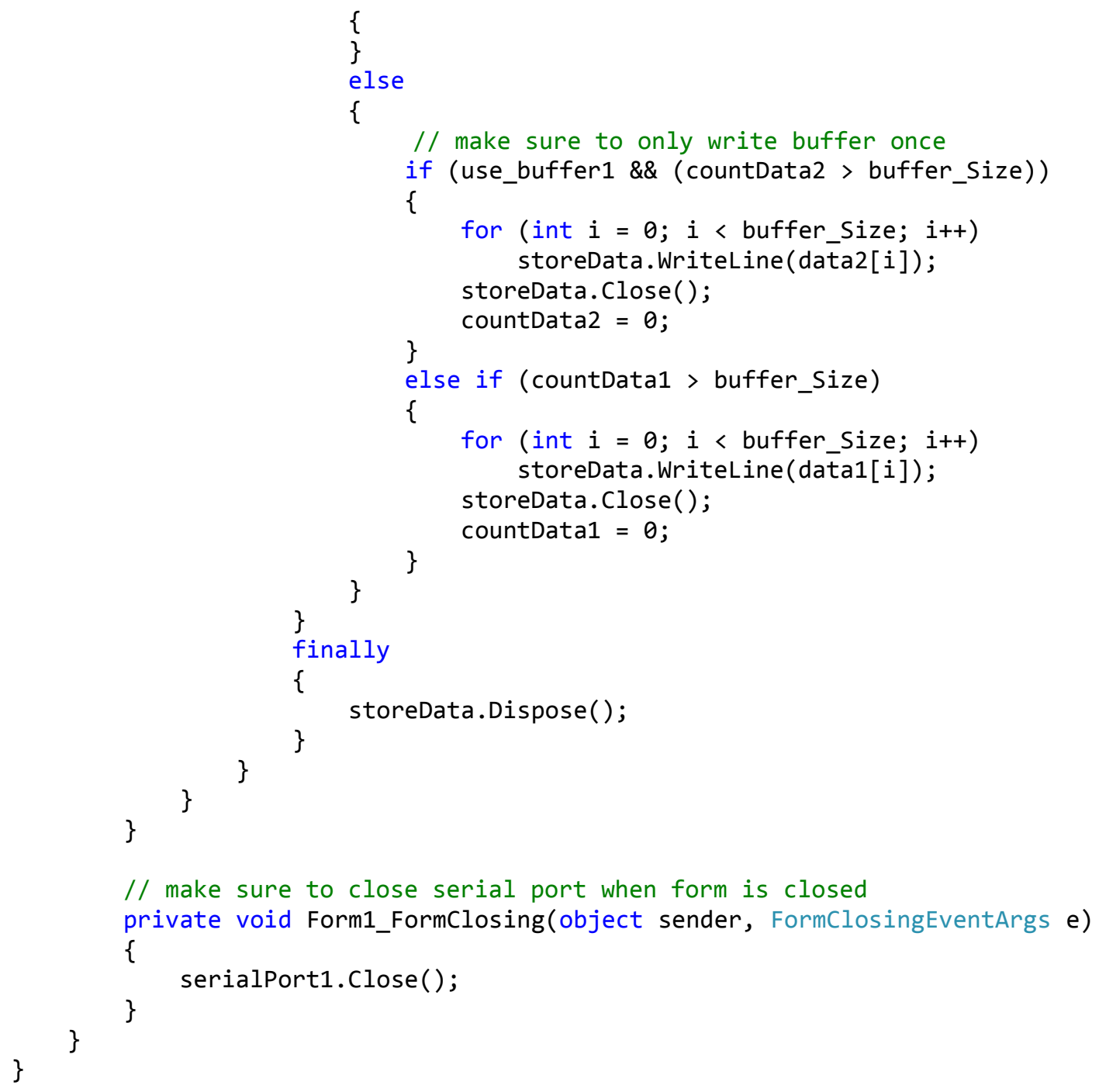

